University of Tennessee Health Science Center

UTHSC Digital Commons

$5-2008$

\title{
The N-terminal Domain of the Y-box Binding Protein YB-1 Plays a Major Role in Cell Proliferation and Apoptosis
}

\author{
Payal Khandelwal \\ University of Tennessee Health Science Center
}

Follow this and additional works at: https://dc.uthsc.edu/dissertations

Part of the Amino Acids, Peptides, and Proteins Commons, and the Medical Sciences Commons

\section{Recommended Citation}

Khandelwal, Payal , "The N-terminal Domain of the Y-box Binding Protein YB-1 Plays a Major Role in Cell Proliferation and Apoptosis" (2008). Theses and Dissertations (ETD). Paper 133. http://dx.doi.org/ 10.21007/etd.cghs.2008.0161.

This Dissertation is brought to you for free and open access by the College of Graduate Health Sciences at UTHSC Digital Commons. It has been accepted for inclusion in Theses and Dissertations (ETD) by an authorized administrator of UTHSC Digital Commons. For more information, please contact jwelch30@uthsc.edu. 


\title{
The N-terminal Domain of the Y-box Binding Protein YB-1 Plays a Major Role in Cell Proliferation and Apoptosis
}

\begin{abstract}
Y-box binding protein, YB-1, is a member of the cold shock domain superfamily of proteins. It is involved in a plethora of cellular functions, including cell proliferation. The molecular mechanisms governing the involvement of YB-1 in cell proliferation are still unclear. Earlier studies done in chicken pre-B lymphocyte DT-40 cells in our laboratory have shown that a targeted disruption in one allele of chicken YB-1

(Chk-Yb-1b) gene at its $\mathrm{N}$-terminal domain resulted in multiple abnormalities in the heterozygous mutants, including slower growth rate, abnormal cell morphology, increased cell size, increased genomic DNA content and significant changes in levels of cell cycle specific genes like the cyclin dependent kinase inhibitor - p21.

The current study is based on the hypothesis that the defects seen in the heterozygous mutant DT-40 cells were due to a dominant negative effect of the potential YB-1 N-terminal truncated protein. The goal of this study is to determine if the introduction of the $\mathrm{N}$-terminal region of YB-1 mimics the defects seen in the mutant DT-40 cells and to identify cell cycle specific proteins that interact with YB-1.

Using rat hepatoma cells (H411E) as a model system, we introduced three different YB-1 N-terminal fusion proteins, i) APYB77GFP, the 77 amino acid long full length YB-1 N-terminal sequence containing clone, ii) APYB36GFP, the 36 amino acid long peptide with the internal deletion $\Delta 12-52$

(APYB36 $12-52$ GFP represented as APYB36GFP) and iii) APYB26GFP, the 26 amino acid proline rich YB-1 $\mathrm{N}$-terminal sequence containing clone and investigated their effects on incubated cells. Our findings show that introduction of the APYB26GFP and APYB77GFP proteins resulted in cell cycle arrest at the G2/M phase. In contrast, incubation with the APYB36GFP protein showed a lower proportion of G2/M phase cells. APYB26GFP and APYB77GFP protein incubations also resulted in significant apoptosis, which was confirmed by Annexin $V$ staining, DNA fragmentation analysis and nuclear breakdown analysis. The APYB36GFP incubation showed lesser percentage of apoptotic cells. We conclude that the YB-1 aminoend sequence plays an important role in cell proliferation and apoptosis.
\end{abstract}

We also demonstrate that YB-1 interacts with the cell cycle protein - cyclin D1, predominantly in the cytoplasm of G2/M phase blocked cells and the YB-1 N-terminus is involved in this interaction. We propose that the proline alanine rich PPAAPPAAPALSAADTK sequence present in APYB26GFP and APYB77GFP, but not in APYB36GFP is involved in this interaction, as cyclin D1 did not immunoprecipitate with APYB36GFP. We conclude that the YB-1 N-terminus interacts with Cyclin D1 in the cytoplasm of G2/ $M$ phase cells and this interaction probably sequesters the cyclin D1 in the cytoplasm leading to cell cycle arrest and apoptosis.

In summary, this study demonstrates that the amino-end domain of YB-1 plays a role in cell proliferation and apoptosis probably by sequestering cyclin D1 in the cell cytoplasm. It is likely that this process is mediated through the proline alanine rich sequence PPAAPPAAPALSAADTK, present in the $\mathrm{N}$-terminus of YB-1.

\section{Document Type}

Dissertation

Degree Name

Doctor of Philosophy $(\mathrm{PhD})$ 


\section{Program}

Molecular Sciences

Research Advisor

Ramareddy V. Guntaka, Ph.D.

\section{Keywords}

YB-1, cell proliferation, apoptosis, transcription

\section{Subject Categories}

Amino Acids, Peptides, and Proteins | Medical Sciences | Medicine and Health Sciences 
The N-terminal Domain of the Y-box Binding Protein YB-1 Plays a Major Role in Cell Proliferation and Apoptosis

\author{
A Dissertation \\ Presented for \\ The Graduate Studies Council \\ The University of Tennessee \\ Health Science Center
}

In Partial Fulfillment

Of the Requirements for the Degree

Doctor of Philosophy

From The University of Tennessee

By

Payal Khandelwal

May 2008 
Copyright (C) 2008 by Payal Khandelwal. All rights reserved. 


\section{Dedication}

This dissertation is dedicated to my mother, Mrs. Hemalata Khandelwal, who has always loved me and believed in me. Through her unending support and constant encouragement I came to understand that I could accomplish any task I set my mind to. 


\section{Acknowledgments}

I am grateful to my research advisor, Dr. Ramareddy V. Guntaka, for giving me the opportunity to do my graduate research in his laboratory. I also want to thank him for his critical evaluation of my scientific writing skills. I wish to thank Drs. Pat Ryan, John Cox, George Hilliard and Rajendra Raghow for being generous enough to be on my committee. I wish to express my deepest gratitude to all of them for their valuable advice, insightful comments, constructive criticism and tremendous help in shaping up and completion of my project. I thank Sudershan Biotech-India for their funding to Dr. R.V. Guntaka, which supported my graduate studies.

I am also very thankful to all my past and present lab mates, especially Drs. German Kamalov, Narina Baburyan, Shalini Thakran and Subramani Koilan for the help, stimulating conversations and valuable suggestions they provided through these years. Their constant co-operation and understanding made this long and arduous process simpler.

I wish to thank Tim Higgins for his immense help in formatting figures for my poster. I also wish to acknowledge the administrative staff of the Department of Molecular Sciences, for their efforts made the graduate school easier.

I would like to thank Dr. David Armbruster and Shirley Hancock for carefully reviewing this dissertation for style and format.

I am extremely thankful to my parents, Mrs. Hemalata Khandelwal and Mr. Anand Mohan Khandelwal, for their constant motivation, love and sacrifice throughout my life. Their unfailing support sustained me through graduate school and made this journey possible. I am also indebted to my husband, Mr. Aseem Maheshwari, for his patience and support. Thanks for always being there. 


\begin{abstract}
Y-box binding protein, YB-1, is a member of the cold shock domain superfamily of proteins. It is involved in a plethora of cellular functions, including cell proliferation. The molecular mechanisms governing the involvement of YB-1 in cell proliferation are still unclear. Earlier studies done in chicken pre-B lymphocyte DT-40 cells in our laboratory have shown that a targeted disruption in one allele of chicken YB-1 (Chk-Yb-1b) gene at its N-terminal domain resulted in multiple abnormalities in the heterozygous mutants, including slower growth rate, abnormal cell morphology, increased cell size, increased genomic DNA content and significant changes in levels of cell cycle specific genes like the cyclin dependent kinase inhibitor - p21.
\end{abstract}

The current study is based on the hypothesis that the defects seen in the heterozygous mutant DT-40 cells were due to a dominant negative effect of the potential YB-1 N-terminal truncated protein. The goal of this study is to determine if the introduction of the N-terminal region of YB-1 mimics the defects seen in the mutant DT-40 cells and to identify cell cycle specific proteins that interact with YB-1.

Using rat hepatoma cells (H411E) as a model system, we introduced three different YB-1 N-terminal fusion proteins, i) APYB77GFP, the 77 amino acid long full length YB-1 N-terminal sequence containing clone, ii) APYB36GFP, the 36 amino acid long peptide with the internal deletion $\Delta 12-52$ (APYB36 $12-52 \mathrm{GFP}$ represented as APYB36GFP) and iii) APYB26GFP, the 26 amino acid proline rich YB-1 N-terminal sequence containing clone and investigated their effects on incubated cells. Our findings show that introduction of the APYB26GFP and APYB77GFP proteins resulted in cell cycle arrest at the G2/M phase. In contrast, incubation with the APYB36GFP protein showed a lower proportion of G2/M phase cells. APYB26GFP and APYB77GFP protein incubations also resulted in significant apoptosis, which was confirmed by Annexin $\mathrm{V}$ staining, DNA fragmentation analysis and nuclear breakdown analysis. The APYB36GFP incubation showed lesser percentage of apoptotic cells. We conclude that the YB-1 amino-end sequence plays an important role in cell proliferation and apoptosis.

We also demonstrate that YB-1 interacts with the cell cycle protein - cyclin D1, predominantly in the cytoplasm of G2/M phase blocked cells and the YB-1 N-terminus is involved in this interaction. We propose that the proline alanine rich PPAAPPAAPALSAADTK sequence present in APYB26GFP and APYB77GFP, but not in APYB36GFP is involved in this interaction, as cyclin D1 did not immunoprecipitate with APYB36GFP. We conclude that the YB-1 N-terminus interacts with Cyclin D1 in the cytoplasm of $\mathrm{G} 2 / \mathrm{M}$ phase cells and this interaction probably sequesters the cyclin D1 in the cytoplasm leading to cell cycle arrest and apoptosis.

In summary, this study demonstrates that the amino-end domain of YB-1 plays a role in cell proliferation and apoptosis probably by sequestering cyclin D1 in the cell cytoplasm. It is likely that this process is mediated through the proline alanine rich sequence PPAAPPAAPALSAADTK, present in the N-terminus of YB-1. 


\section{Table of Contents}

Chapter 1. Introduction............................................................................................................1

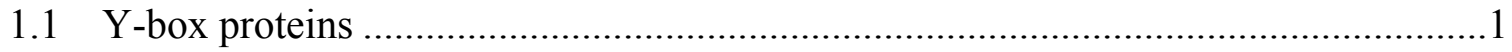

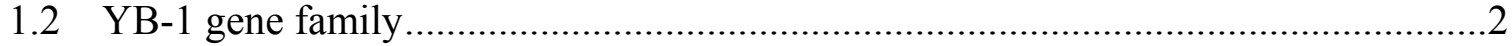

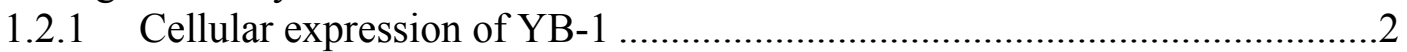

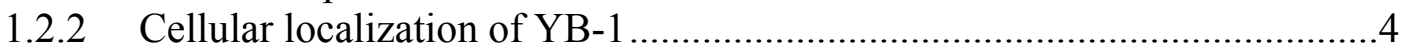

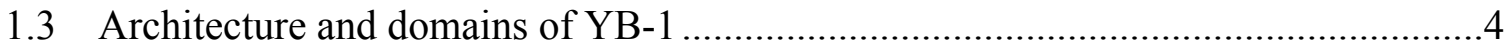

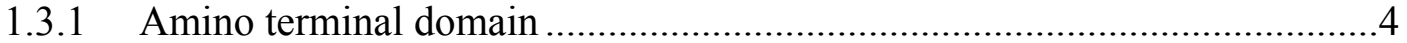

1.3.2 Cold shock/nucleic acid binding domain ..................................................

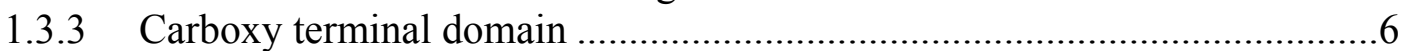

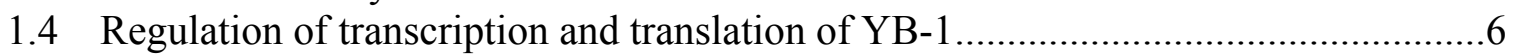

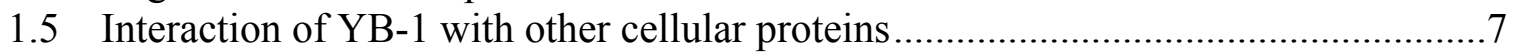

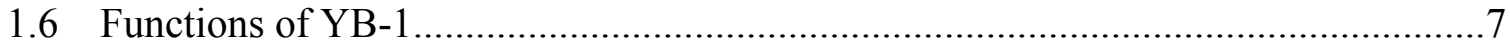

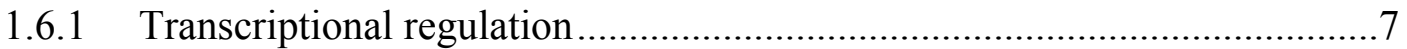

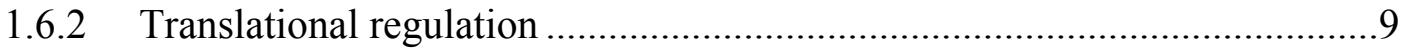

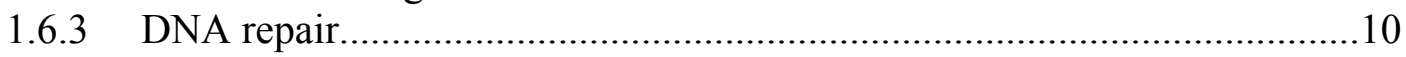

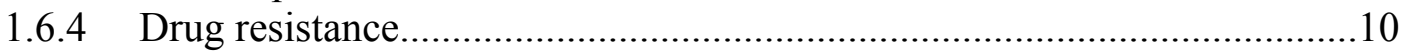

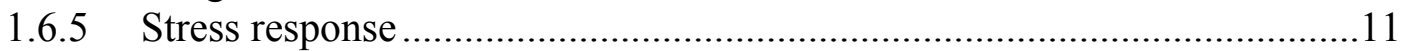

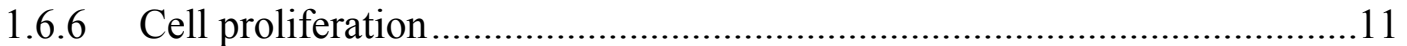

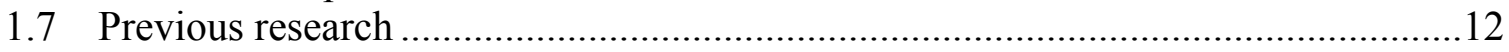

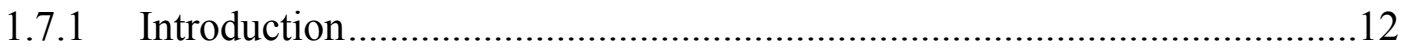

1.7.2 Strategy used for disruption of one allele of Chk-Yb-1b gene ...................13

1.7.3 Defects seen in the heterozygous mutant DT-40 cells .................................13

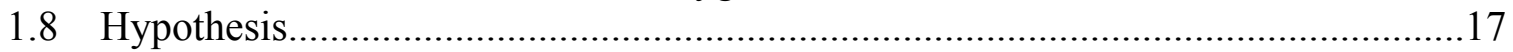

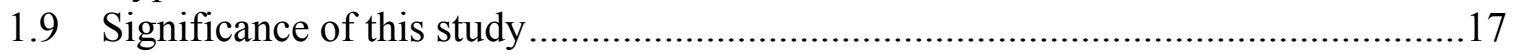

Chapter 2. YB-1 Amino Terminal Domain Plays a Major Role in Cell Proliferation and Apoptosis ....................................................................................................18

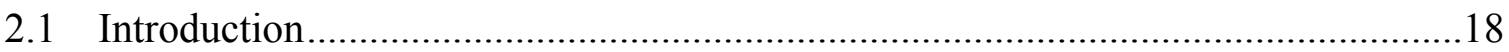

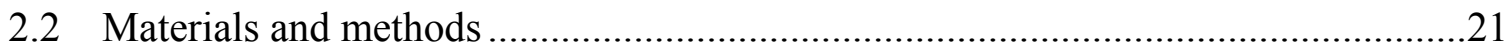

2.2.1 Antibodies and reagents ...................................................................21

2.2.2 Cloning and expression of APGFP-fusion proteins...................................21

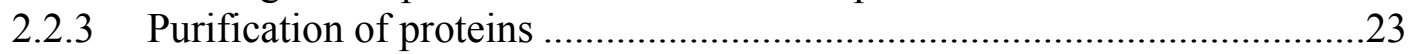

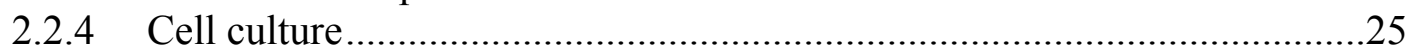

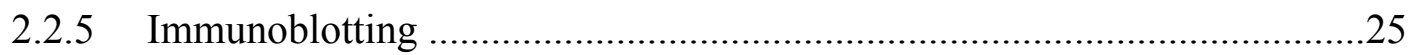

2.2.6 Immunocytochemistry and indirect immunofluorescence .........................26

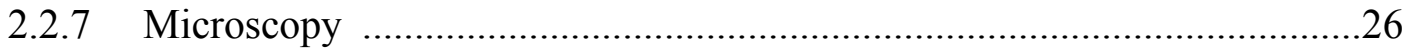

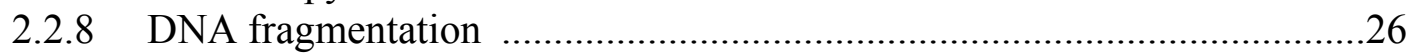

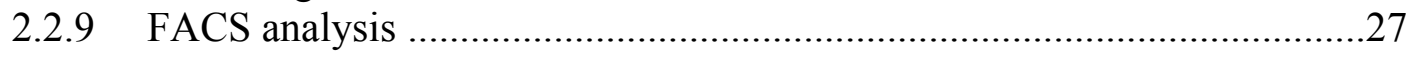

2.2.10 Quantitation of immunoblotting and immunofluorescence data ................27

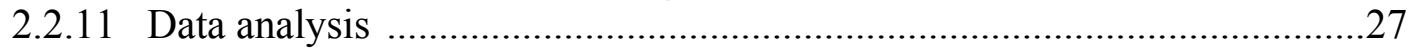


2.3.1 APYBGFP and APGFP fusion proteins get internalized and are stable inside the cells .........................................................................28

2.3.2 YB-1 sequence appears to restrict to cell cytoplasm ...............................28

2.3.3 Effect of external stimuli on the localization of APYBGFP proteins..........31

2.3.4 Effect of APYBGFP proteins on cell cycle .............................................36

2.3.5 YB-1 amino terminal domain is involved in significant apoptosis.............40

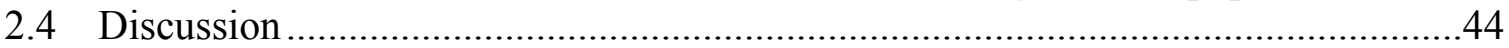

\section{Chapter 3. YB-1 Interacts with Cyclin D1 in the Cytoplasm of Cells in} G2/M Phase of Cell Cycle.

3.1 Introduction 49

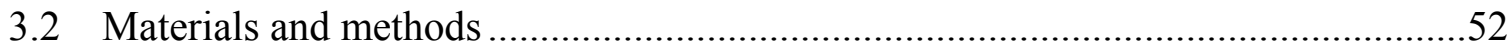

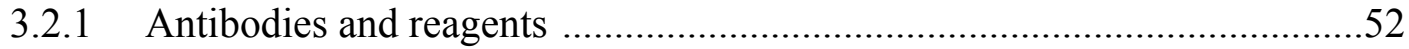

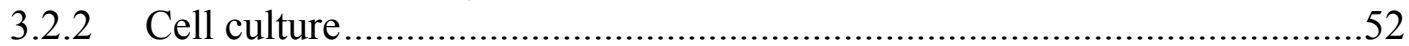

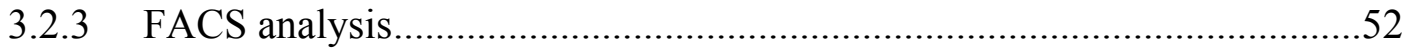

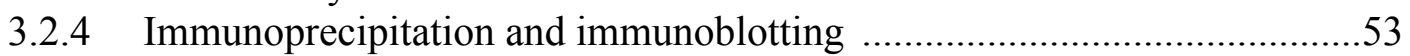

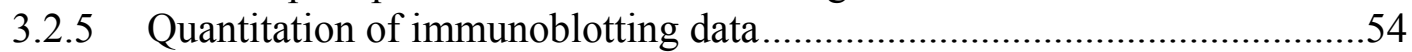

3.2.6 Immunocytochemistry and indirect immunofluorescence.......................54

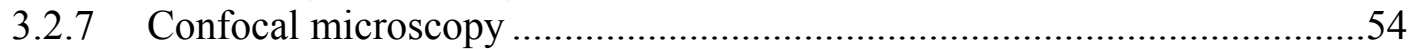

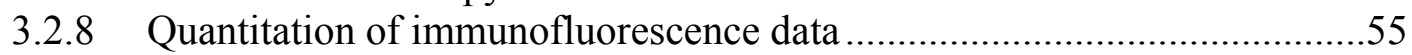

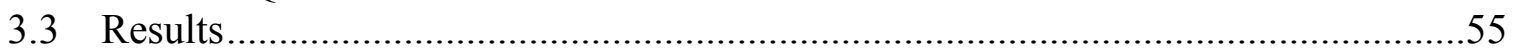

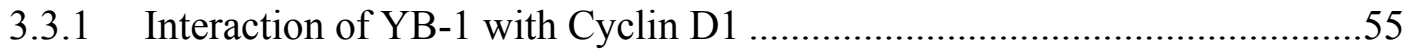

3.3.2 YB-1 co-localizes with Cyclin D1 predominantly at the G2/M phase .......55

3.3.3 Quantitative interaction of YB-1 with Cyclin D1 and vice versa ..............62

3.3.4 N-terminus of YB-1 is involved in the interaction of YB-1

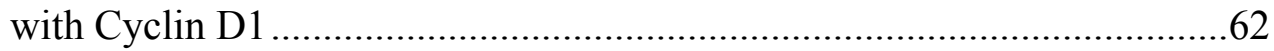

3.3.5 Inhibition of YB-1 phosphorylation by Wortmannin results in re-localization of Cyclin D1 in the nucleus even after nocodazole block

3.3.6 Inhibition of Cyclin D1 phosphorylation does not change co-localization of YB-1 with Cyclin D1 ................................................68

3.3.7 YB-1 Cyclin D1 interaction is not completely mediated by RNA .............68

3.3.8 YB-1 and Cyclin D1 show a change in distribution pattern after

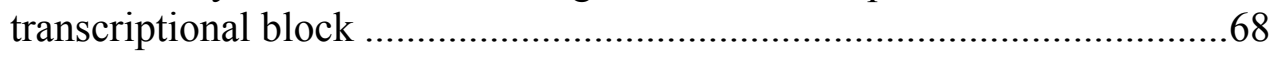

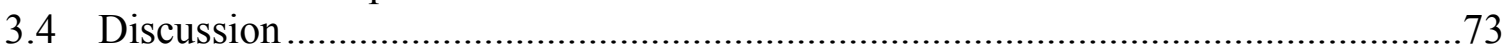

Chapter 4. Summary and Overview......................................................................81

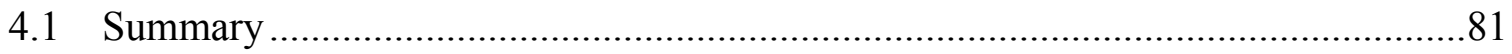

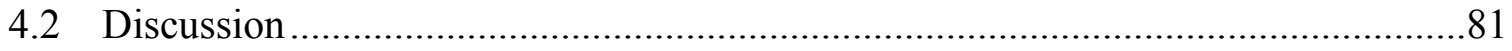

4.2.1 Potential YB-1 N-terminal sequences playing a role in cell proliferation. 
4.2.2 YB-1 N-terminus interacts with cyclin D1 in the cytoplasm of G2/M

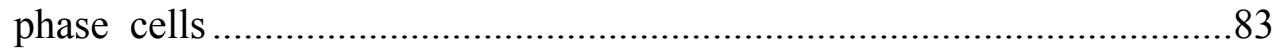

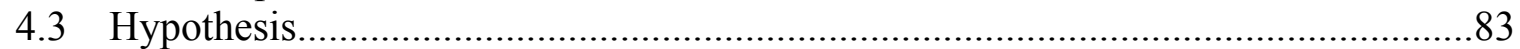

4.4 Future experiments to test our hypothesis …………..........................................85

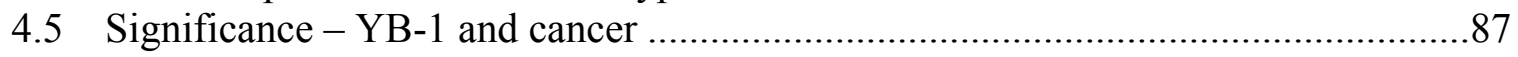

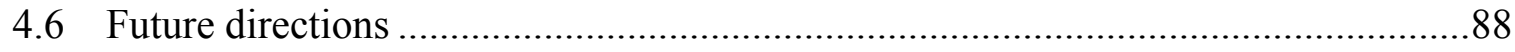

List of References .....................................................................................................................90

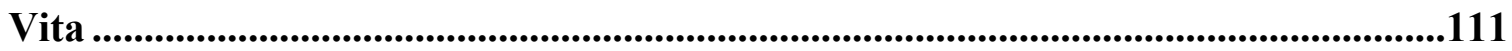




\section{List of Tables}

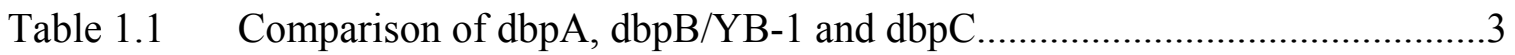

Table 2.1 GFP positive cell \% in cell cycle assay as determined by FACS analysis

Table 2.2 Mean GFP fluorescence in cell cycle assay as determined by FACS analysis

Table 2.3 GFP positive cell \% in apoptotic assay as determined by FACS analysis

Table 2.4 Mean GFP fluorescence in apoptotic assay as determined by FACS analysis

Table 3.1 Quantitative co-localization of YB-1 with Cyclin D1 and vice versa in control cells. .64

Table 3.2 Quantitative co-localization of YB-1 with Cyclin D1 and vice versa in nocodazole blocked cells 


\section{List of Figures}

Figure 1.1 Schematic representation of the amino acid alignment of $\mathrm{dbpA}$, $\mathrm{dbpB} / \mathrm{YB}-1$ and $\mathrm{dbpC}$

Figure 1.2 Schematic representation of some of the YB-1 protein interacting partners and the domain of YB-1 involved in this interaction....................8

Figure 1.3 Schematic representation of disruption of one allele of YB-1 gene

Figure 1.4 Schematic representation of splicing of the two alleles of Chk-YB-1 gene after the disruption of one allele.

Figure 1.5 Defects seen in the heterozygous mutant DT-40 cells

Figure 2.1 Comparison of disruption made in the YB-1 domains by various investigators and their corresponding defective functions.

Figure 2.2 Strategy of cloning the APGFP fusion proteins...................................22

Figure 2.3 YB-1 N-terminal amino acid sequences used for cloning .......................22

Figure 2.4 Purified fusion proteins on a SDS-PAGE gel .....................................24

Figure 2.5 Schematic representation of the four clones and their sizes on a SDS-PAGE Western blot.

Figure 2.6 APYBGFP and APGFP fusion proteins get internalized and are stable inside the cells

Figure 2.7 APYBGFP fusion proteins are localized in cell cytoplasm. 30

Figure 2.8 Cytoplasmic localization of the APYBGFP fusion proteins confirmed by western blotting

Figure 2.9 Localization of the APYBGFP proteins in rat hepatoma cells changes in response to different stimuli

Figure 2.10 APYBGFP fusion proteins partially translocated to the nucleus following $20 \%$ FBS treatment

Figure 2.11 APYB77GFP gets partially translocated to the nucleus under UV treatment. 
Figure 2.12 APYBGFP fusion proteins are stable until the $72 \mathrm{hrs}$. time point of incubation

Figure 2.13 Cells incubated with the APYBGFP proteins show cell cycle arrest at the $\mathrm{G} 2 / \mathrm{M}$ phase of cell cycle

Figure 2.14 APYBGFP protein incubation results in significant apoptosis by Annexin V staining and FACS analysis

Figure 2.15 APYBGFP protein incubation results in significant DNA fragmentation (Karyorrhexis).

Figure 2.16 APYBGFP protein incubation results in significant pyknosis

Figure 3.1 YB-1 and Cyclin D1 co-immunoprecipitate with each other in rat hepatoma cells .56

Figure 3.2 Synchronization of cells at the G1/S phase of cell cycle by a double thymidine block

Figure 3.3 Synchronization of cells at the G2/M phase of cell cycle by a nocodazole block

Figure 3.4 Co-localization of YB-1 and Cyclin D1 in G2/M phase

Figure 3.5 Interaction of YB-1 with Cyclin D1 and vice-versa is seen predominantly at the $\mathrm{G} 2 / \mathrm{M}$ phase of cell cycle

Figure 3.6 YB-1 immunoprecipitates approximately $80 \%$ of the total Cyclin D1 and vice versa at the $\mathrm{G} 2 / \mathrm{M}$ phase of cell cycle.

Figure 3.7 APYB26GFP and APYB77GFP immunoprecipitate Cyclin D1 in $\mathrm{G} 2 / \mathrm{M}$ phase blocked rat hepatoma cells

Figure 3.8 Inhibition of YB-1 phosphorylation by Wortmannin resulted in re-localization of Cyclin D1 in the nucleus even after nocodazole block

Figure 3.9 Inhibition of Cyclin D1 phosphorylation with $\mathrm{LiCl}$ did not change the co-localization of YB-1 and Cyclin D1 in G2/M phase blocked cells.

Figure 3.10 Immunoblot analysis of nuclear and cytoplasmic extracts of cells incubated with $\mathrm{LiCl}$ and/or nocodazole. 
Figure 3.11 Lamin is seen only in nuclear extracts of cells treated with nocodazole

Figure 3.12 Evidence that nocodazole treatment does not cause breakdown of nuclear membrane..............................................................................

Figure 3.13 YB-1 and Cyclin D1 interaction is independent of mRNA........................72

Figure 3.14 YB-1 and Cyclin D1 show a change in distribution after actinomycin D treatment.........................................................................74

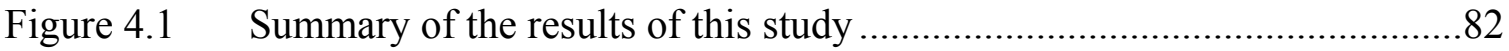

Figure 4.2 Model of cyclin D1 sequestration by binding to YB-1 N-terminal proteins in the cytoplasm of $\mathrm{G} 2 / \mathrm{M}$ phase cells leading to cell cycle

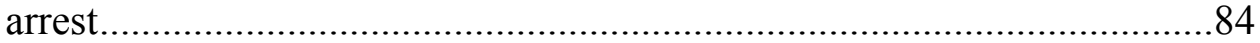




\section{List of Abbreviations}

APGFP Fusion protein with antennapedia and GFP sequences APYB26GFP Fusion Protein with Proline Rich 26 amino acid sequence APYB36GFP Fusion protein with internal deletion of 11-52 aminoacids APYB77GFP. Fusion Protein with full length YB-1 N-terminal 77 amino acids BCA Bicinchoninic acid

BSA Bovine serum albumin

Chk-Yb-1b Chicken YB-1

CDK Cyclin dependent kinase

DAPI 4',6-diamidino-2-phenylindole

DMEM

DMSO Dimethyl sulfoxide

DT -40

E. coli Chicken pre B lymphocyte cells

FACS Escherichia coli

FBS Fluorescence activated cell sorting FITC Fetal bovine serum GFP Fluorescein isothiocynate

GSK-3 $\beta$

MEM

MHC Dulbecco's modified eagle's medium

mRNA mRNP.

$\mathrm{NF}-\kappa \mathrm{B}$ Green fluorescent protein

PAGE

PCNA

PBS Glycogen synthase kinase - $3 \beta$ Minimum essential medium

PI Major histocompatibility

PI-3K Messenger RNA

PMSF

SDS Messenger ribonucleoprotein Nuclear factor-kappa B siRNA

YB-1 Polyacrylamide gel electrophoresis . Proliferating cell nuclear antigen . Phosphate buffered saline Propidium iodide Phosphoinositide-3-kinase Phenylmethylsulphonyl fluoride Sodium dodecyl sulphate Small interference RNA Y-box binding protein-1 


\section{Chapter 1. Introduction}

All living organisms must adapt to changes in the environment. One of the most primitive forms of environmental change that an organism can be exposed to is a change in temperature. All organisms have developed various essential adaptive mechanisms to survive within a wide range of temperature stresses (Thieringer et al., 1998). The cellular response to the temperature stress is represented at the molecular level by the synthesis of heat-shock or cold-shock proteins. Heat shock proteins are found in bacteria, yeast, plants and animals. They generally function as molecular chaperones or proteases and their structure and functions are conserved in all organisms (Lindquist, 1986). In contrast, cold shock proteins or cold induced proteins found in bacteria function as RNA chaperones (Matsumoto et al., 1998).

The nucleic acid binding region present in eukaryotic cold shock proteins is homologous to the bacterial cold shock proteins (Csps). Therefore, proteins having the nucleic acid binding region belong to the cold shock domain (CSD) superfamily. Y-box binding proteins which bind to the inverted CCAAT box on gene promoters belong to the cold shock domain superfamily of proteins. CSD proteins have not been reported in yeast as yet. All proteins in the CSD family contain a $\mathrm{N}$-terminal and a $\mathrm{C}$-terminal domain, which are thought to have been added during evolution from unicellular to multicellular organisms.

\subsection{Y-box proteins}

The Y-box family of proteins is perhaps the family of most evolutionarily conserved nucleic acid binding proteins from plants to vertebrates. But the structure of the $\mathrm{N}$ and $\mathrm{C}$-terminal domains of various $\mathrm{Y}$-box proteins vary significantly. These alterations might have been necessary for the evolution from poikilotherms to homiotherms and may be involved in its multiple functions. The Y-box proteins were first identified and isolated by binding to a DNA probe containing the Y-box sequence 5'-CTGATTGGCCAA-3' (Dorn et al., 1987). The cloning and characterization of the Y-box transcription factor from Xenopus laevis encoded sequence specific DNA binding domains called FRGY1 and FRGY2 (Murray et al., 1992). These sequences were found in oocytes, embryos and adult tissues (Tafuri et al., 1990). The cDNA clone for the CCAAT transcription factor EF1A also revealed novel structural motifs containing

alternative stretches of positively and negatively charged amino acids (Ozer et al., 1990).

Proteins of this family are active as transcriptional and translational regulators for a wide array of genes containing the Y-box sequence in their promoters (Ladomery, 1997) and for messenger RNA. Initial studies suggested that binding of the Y-box proteins to the intact Y-box sequence involves specific interactions (Didier et al., 1988). Later, binding studies showed that Y-box proteins can bind to a wide range of nucleic acids, like single stranded DNA (ssDNA), damaged DNA and even triple stranded DNA and RNA (Horwitz et al., 1994; Koike et al., 1997). Y-box proteins have a special preference for 
binding ssDNA, especially when it contains the Y-box sequence (Tafuri et al., 1992).

They also show a preference for binding pyramidine rich DNA sequences (Wolffe, 1994).

\subsection{YB-1 gene family}

Y-box binding protein YB-1 is a member of the Y-box protein family. It was first named in 1988 with reference to the transcription factors binding to the Y-box of MHC class II promoters (Didier et al., 1988). YB-1 protein was also found to interact with the HER-2 promoter and EGFR enhancer around the same time (Sakura et al., 1988). It binds to an inverted CCAAT box (Y-box) repeat within the consensus sequence 5'-CTGATTGG-3', present in the promoter region of the genes it regulates (Ladomery, 1997).

The human YB-1 cDNA was first isolated by screening a human lymphoblastoid cell cDNA library expressed in Escherichia coli with an oligodeoxynucleotide containing the Y-box sequence (Didier et al., 1988). In due course, additional Y-box binding proteins were identified in frogs (Tafuri et al., 1990), rats (Ozer et al, 1990), mice (Tafuri et al., 1993) and chickens (Grant et al., 1993; Kandala and Guntaka, 1994). A number of genes closely related to YB-1 have also been identified, including dbpA cloned in 1995 (Kudo et al., 1995) and contrin or dbpC cloned in 1999 (Tekur et al., 1999). Comparison of characteristics of these genes is shown in Table 1.1. YB-1 is identical to dbpB. Several eukaryotic genes including genes encoding major histocompatibility class II antigens, thymidine kinase, proliferating cell nuclear antigen, DNA polymerase $\alpha$, epidermal growth factor receptor, DNA topoisomerase II $\alpha$ and multidrug resistance I protein contain the Y-box binding consensus sequence in their regulatory regions (Wolffe et al., 1992 and 1994; Ladomery et al., 1995).

YB-1 gene is comprised of 8 exons and 7 introns spanning $19 \mathrm{~kb}$ of genomic DNA and is located on chromosome $1 \mathrm{p} 34$ (Toh et al., 1998). All introns are flanked by the dinucleotides GT and AG, consistent with the eukaryotic gene sequences for splice junctions in eukaryotic genes (Breathnach et al., 1981). The YB-1 mRNA is approximately $1.5 \mathrm{~kb}$ long and codes for a 324 amino acid protein with a predicted mass of $43 \mathrm{kDa}$. YB-1 CSD is encoded by exons 1-5 and C-terminal domain by exons 6-8. Sequence diversity among different YB-1 cDNA clones has also been described (Kolluri et al., 1991).

\subsubsection{Cellular expression of $Y B-1$}

YB-1 is induced in various cell types in response to mitogenic stimuli, such as cytokine stimulated T-cells (Sabath et al., 1990), serum activated fibroblasts (Ito et al., 1994) and agonist stimulated endothelial cells (Stenina et al., 2000). It is ubiquitously expressed, especially in skeletal muscles, kidney, lungs and liver. Actively proliferating cells/tissues like early chicken and rat embryos (Grant et al., 1993; Ito et al., 1994), wound healing cells in regenerating liver tissue following chemical induced damage 
Table 1.1 Comparison of $\operatorname{dbp} A, \mathrm{dbpB} / \mathrm{YB}-1$ and $\mathrm{dbpC}$

\begin{tabular}{cccccc}
\hline Name & Chromosome & Gene (kbp) & No. of exons & Amino Acids & Expression \\
\hline $\mathrm{dbpA}$ & $12 \mathrm{p} 13$ & 24 & 10 & 372 & Heart, Muscle \\
$\mathrm{dbpB} /$ YB-1 & $1 \mathrm{p} 34$ & 19 & 8 & 324 & Ubiquitous \\
$\mathrm{dbpC}$ & $17 \mathrm{p} 11$ & 6 & 9 & 364 & Germ cell \\
\hline
\end{tabular}

Comparison of characteristics of the members of the human Y-box protein family. (Adapted with permission. Kohno, K., Izumi, H., Uchiumi, T., Ashizuka, M., and Kuwano, M. (2003) The pleiotropic functions of the Y-box-binding protein, YB-1. Bioessays. 25, 691-698.) 
(Grant et al., 1993), or hepatectomy (Ito et al., 1994), colorectal epithelial glands (Shibao et al., 1999) and various cancer cells (Ohga et al., 1996) show higher expression of YB-1 as compared to quiescent/adult differentiated cells.

\subsubsection{Cellular localization of YB-1}

YB-1 is a major structural component of messenger ribonucleoprotein (mRNP) complexes in the cells. It is predominantly present in the cell cytoplasm. It is translocated to the nucleus under conditions like U.V. irradiation (Koike et al., 1997), hyperthermia (Stein et al., 2001), IF- $\gamma$ treatment (Higashi et al., 2003), adenovirus infection (Holm et al., 2002; Paranjape et al., 2007), along with splicing factor SRp30c (Raffetseder et al., 2003) and under high levels of ectopic YB-1 expression (Zhang et al., 2006). Both the CSD and CT domains of YB-1 are implicated in its nuclear localization. The CT domain consists of a non-canonical nuclear localization signal and the CSD is shown to contribute to the nuclear retention (Bader et al., 2005). However the CT domain can also bind to a yet unknown anchoring protein in the cytoplasm and thus retain the YB-1 there (Koike et al., 1997). YB-1 is also shown to associate with nascent transcripts cotranscriptionally and is presumed to accompany mRNA into the cytoplasm (Soop et al., 2003).

\subsection{Architecture and domains of YB-1}

The vertebrate Y-box proteins are comprised of three domains, the variable amino or N-terminal domain, a highly conserved nucleic acid binding domain or the cold shock domain (CSD) and a carboxyl or C-terminal domain (Wolffe et al., 1994; Graumann et al., 1998). A comparison of the three well-characterized Y-box proteins is shown in Fig. 1.1 .

\subsubsection{Amino terminal domain}

The YB-1 N-terminal domain is rich in amino acids alanine and proline. Functionally, the YB-1 N-terminal domain has not been very well defined, though it is thought to be a trans-activation/regulation domain (Tafuri et al., 1992; Wolffe, A.P., 1994; Kohno et al., 2003). YB-1 also binds to some proteins through its N-terminal domain, for example, p53 (Okamoto et al., 2000).

\subsubsection{Cold shock/nucleic acid binding domain}

The YB-1 CSD domain, consisting of approximately 80 amino acids, is one of the most evolutionary conserved domains from bacteria to eukaryotes (Wolffe et al., 1992; Wolffe, 1994; Ladomery et al., 1995). It shows 93\% sequence identity within the family of Y-box proteins (Wolffe, 1994). Structurally, it is comprised of a five-stranded $\beta$-barrel 
$\operatorname{dbpA}$

\begin{tabular}{|l|l|l|}
\hline \multicolumn{1}{|c|}{83} & 161 & 372 \\
\hline A/P & CSD & B/A repeats \\
\hline
\end{tabular}

dbpB/Y B-1

\begin{tabular}{|l|l|l|}
\hline \multicolumn{1}{|c}{51} & & \\
\hline A/P & CSD & B/A repeats \\
\hline
\end{tabular}

\begin{tabular}{l|l|l|l|} 
& \multicolumn{1}{c}{86} & \multicolumn{1}{c}{164} \\
$\mathrm{dbpC} /$ contrin & $\mathrm{A} / \mathrm{P}$ & $\mathrm{CSD}$ & $\mathrm{B} /$ A repeats \\
\hline
\end{tabular}

Fig. 1.1 Schematic representation of the amino acid alignment of $\operatorname{dbp} A, \operatorname{dbp} B / Y B-1$ and dbpC

This figure depicts the organization of domains of three members in the CSD superfamily of proteins. $\mathrm{A} / \mathrm{P}$ indicates the alanine and proline rich $\mathrm{N}$-terminal domain and $\mathrm{B} / \mathrm{A}$ repeat indicates the basic and acidic amino acid clusters in the $\mathrm{C}$-terminal domain. (Adapted with permission. Kohno, K., Izumi, H., Uchiumi, T., Ashizuka, M., and Kuwano, M. (2003) The pleiotropic functions of the Y-box-binding protein, YB-1. Bioessays. 25, 691698.) 
(Wistow et al., 1990) containing RNP-1 and RNP-2 like consensus motifs (Landsman, 1992) and belongs to the $\beta$-sheet RNA-binding protein group (Graumann et al., 1996).

The CSD domain is involved in binding to DNA and RNA (Wolffe et al., 1992 and 1994; Schindelin et al., 1994; Bouvet et al., 1995). It is also involved in specific (Bouvet et al., 1995) and non-specific RNA binding (Tafuri et al., 1993) and in specific ssDNA binding (Tafuri et al., 1992). The non-specific RNA binding occurs during packaging of mRNA in maturing germ cells. The specific RNA binding takes place in the nucleation process during the assembly of mRNP particles (Wolffe et al., 1994; Bouvet et al., 1995). The specific ssDNA binding is required for the binding of YB-1 to specific single stranded parts of promoters, which thereby affect the binding of other transcription factors and induce transcription (MacDonald et al., 1995; Swamynathan et al., 1998).

\subsubsection{Carboxy terminal domain}

The YB-1 C-terminal domain consists of alternating clusters of about 30 acidic and basic amino acids, referred to as B/A islands (Murray et al., 1992). Structurally, the acidic regions separating the $\mathrm{B} / \mathrm{A}$ islands are predicted to form alpha helices. The $\mathrm{B} / \mathrm{A}$ repeats may be a structure unique to vertebrate organisms, since plants and invertebrates show zinc finger motifs.

The YB-1 C-terminal domain binds DNA and RNA in a sequence-independent manner (Izumi et al., 2001). The structure of this domain helps it function as a charged zipper, facilitates dimer formation (Tafuri et al, 1992) and is essential for the formation of messenger ribonucleoprotein (mRNP) particles in which mRNA is stored (Matsumoto et al., 1996). The C-terminal domain is also thought to be involved in protein-protein interactions (Bouvet et al., 1995).

\subsection{Regulation of transcription and translation of YB-1}

The levels of YB-1 protein have to be precisely regulated for cells to function normally. YB-1 production is regulated both at transcriptional and posttranscriptional/translational levels. YB-1 gene promoter has multiple E-boxes and GC-boxes (Makino et al., 1996). Transcription of YB-1 gene is stimulated by the tumor suppressor gene product, p73, through an enhanced recruitment of the c-Myc-Max complexes to the E-Box sequences (Uramoto et al., 2002). YB-1 mRNA shows regulation by its own product. YB-1 protein represses translation of YB-1 mRNA by binding to specific elements in the 5' and 3' untranslated regions (Fukuda et al., 2004; Skabkina et al., 2005). YB-1 protein is negatively regulated by F-Box protein 33 (FBX33), which targets the YB-1 protein for polyubiquitination and proteosomal degradation (Lutz et al., 2006). 


\subsection{Interaction of YB-1 with other cellular proteins}

YB-1 interacts with a diverse range of proteins, which are shown in Fig. 1.2. It interacts with other transcription factors like p53 (Okamoto et al., 2000), NFкB sub unit Rel A or p65 (Raj et al., 1996), AP-2 (Mertens et al., 2002), multivalent zinc finger factor - CTCF (Chernukhin et al., 2000), TBP (Shnyreva et al., 2000), Smad3 (Higashi et al., 2003) and YY-1 (Matsumoto et al., 2005). It also shows interaction with some RNA binding proteins like EWS (Chansky et al., 2001), AUF-1 (Moraes et al., 2003), Poly (C)binding protein (Funke et al., 1996), SRp30 (Raffetseder et al., 2003), exuperantia (Wilhelm et al., 2000), iron responsive element binding protein-2 or IRP2 and hnRNPK (Ashizuka et al., 2002; Shnyreva et al., 2000) and YBAP1 (Matsumoto et al., 2005). Cell cycle proteins like Cdk4 (Balda et al., 2003) and Cdk5 (Moorthamer et al., 1999), repair proteins like proliferating cell nuclear antigen - PCNA (Ise et al., 1999) and human endonuclease III (Marenstein et al., 1999) also interact with YB-1. Among the other protein physical partners of YB-1 are viral proteins like human immunodeficiency type 1 tat protein (Ansari et al., 1999), TAR RNA molecules and human polyoma JC virus large T antigen (Safak et al., 1999). YB-1 also interacts with the proteins ZO-1 (Balda et al., 2000), RalA (Frankel et al., 2005), Pur- $\alpha$ (Safak et al., 1999), DNA binding protein A and cardiac ankyrin repeat protein - CARP (Zou et al., 1997) and actin filaments (Ruzanov et al., 1999). These interactions indicate the complexity involved in the expression of pleiotropic functions of YB-1. Further characterization of these interactions will aid in a better understanding of the different integrated functions of YB-1.

\subsection{Functions of YB-1}

Y-box binding protein YB-1 is a multifunctional DNA/RNA binding protein, which is involved in reproduction, storing and expression of genetic information. It is shown to have a role in varied cellular functions like transcription and translation regulation, DNA repair, drug resistance, stress response and regulation and cell proliferation.

\subsubsection{Transcriptional regulation}

YB-1 controls the transcription of various eukaryotic genes involved in different cellular functions, especially cell proliferation and DNA repair. Transcription regulation by YB-1 can be classified into three main categories.

YB-1 can bind directly to the Y-box or related sequences in the promoter regions of many genes and regulate transcription. For example, YB-1 was shown to directly bind to the Y-box motif in cyclin A promoter and to the proximal and distal CCAAT boxes in

cyclin B1 promoter by electrophoretic mobility shift assay. Using reporter gene construct analysis, this binding was shown to directly regulate the transcription of both the cyclin genes (Jurchott et al., 2003). Similarly transcription regulation is seen, by the direct binding of YB-1 to the Y-box in promoter regions of genes like MDR1 (Swamynathan et 


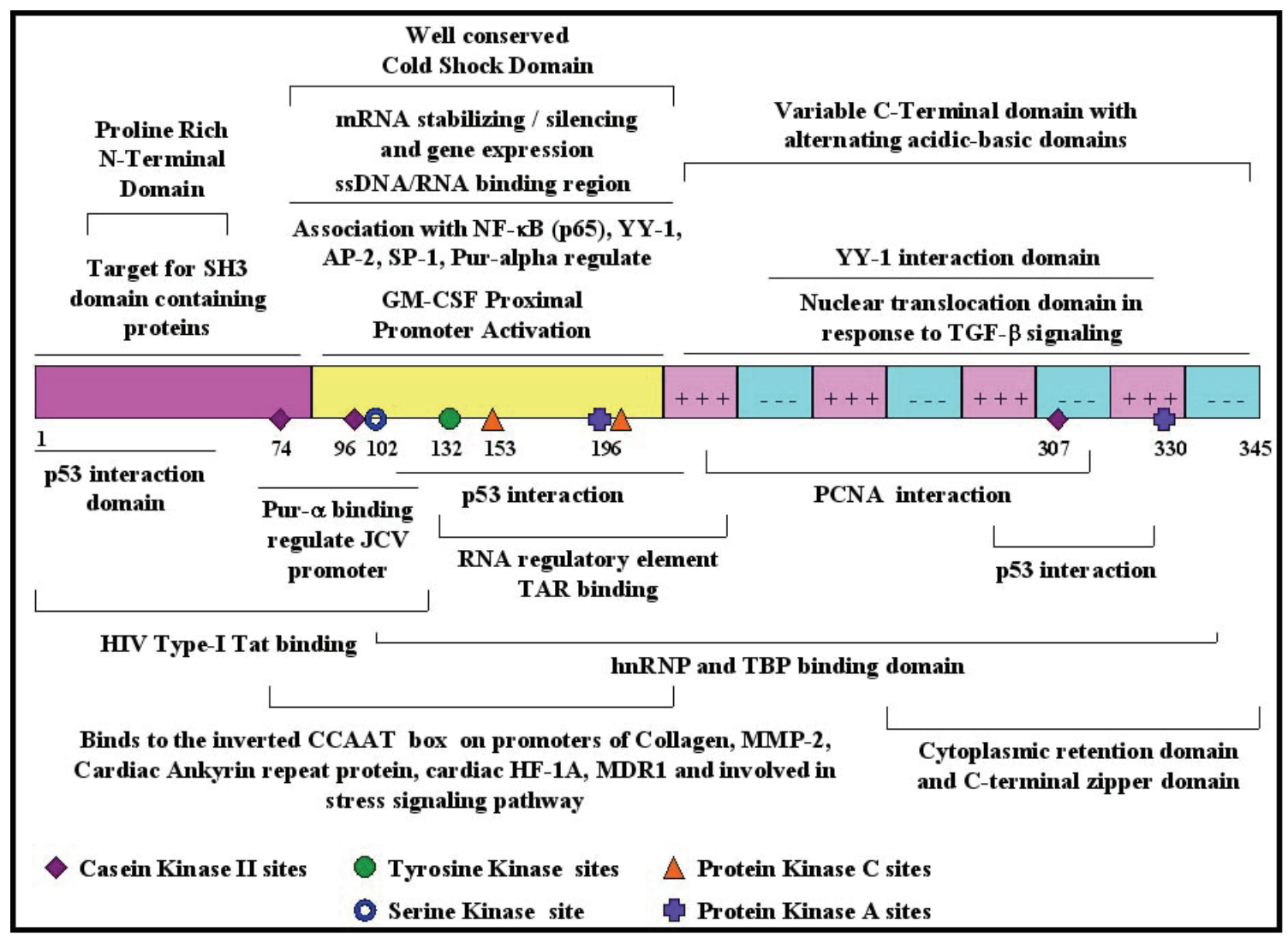

Fig. 1.2 Schematic representation of some of the YB-1 protein interacting partners and the domain of YB-1 involved in this interaction

This is a non-exhaustive representation of interaction of YB-1 with other proteins. YB-1 is shown to interact with a multitude of proteins through one or more domains; for example, it interacts with $\mathrm{p} 53$ through all three domains, whereas only the C-terminal domain mediates its interaction with PCNA. 
al., 1998), human major vault protein (MVP) or the lung resistance related protein (LRP) (Stein et al., 2005), myosin light-chain $2 \mathrm{v}$ (Zou et al., 1995), human $\alpha 2$ collagen (Higashi et al., 2003) and MMP13 (Samuel et al., 2007).

YB-1 can also regulate gene expression in a Y-box independent manner, via interaction with other transcriptional factors. For example, co-transfection analysis showed that YB-1 and Rel A / NF- $\mathrm{kB}$ p65 subunit cooperate to synergistically activate the GM-CSF proximal promoter (Diamond et al., 2001). Similar transcription regulation is seen in genes like p21 (Okamoto et al., 2000) and grp ${ }^{73}$ (Li et al., 1997) among others.

YB-1 can also bind to single stranded DNA with high affinity for transcription regulation. Presence of single stranded DNA has been reported in regions of many transcriptionally active promoters (Swamynathan et al., 1998; Wilusz et al., 2001). By radiolabeled single stranded probe analysis YB-1 was shown to bind preferentially to single stranded components on MMP-2 promoter and by repoter gene constructs this binding was shown to regulate transcription of the MMP-2 (Mertens et al., 1997). This mode of transcriptional regulation by YB-1 occurs in other genes also like HIV-1 (Sawaya et al., 1998), Fas/CD95 (Lasham et al., 2000), Rat $\alpha 1$ procollagen (Dhalla et al., 1998), rat gelatinase A (Mertens et al., 1999 and 2002), JC polyomavirus late gene (Chen et al., 1995; Safak et al., 1999), MHC class II genes (MacDonald et al., 1997; Montani et al., 1998), thyrotropin receptor (Ohmori et al., 1996), mouse $\alpha 1$ collagen (Norman et al., 2001), GM-CSF (Coles et al., 2000), VEGF (Coles et al., 2002) and EGFR (Stratford et al., 2007)

\subsubsection{Translational regulation}

Translation regulation of genes by YB-1 is mediated by different mechanisms. It binds with mRNA nuclear precursors and thus participates in alternative splicing of mRNA (Chansky et al., 2001; Stickeler et al., 2001). In the cytoplasm of cells, YB-1 serves as the main mRNA packaging protein (Skabkin et al., 2004) where it regulates the lifetime (Evdokimova et al., 2001) and mRNA template activity in protein synthesis (Minich et al., 1992; Sommerville et al., 1999; Evdokimova et al., 1999). It has been shown to have a dual effect on protein synthesis. At low ratios typical for polysomal mRNPs, YB-1 stimulates translation at the stage of initiation (Minich et al., 1992; Evdokimova et al., 1998; Pisarev et al., 2002). At an increased ratio corresponding to free mRNPs, YB-1 inhibits the protein synthesis both in vitro (Minich et al., 1992; Nekrasov et al., 2003) and in vivo (Davydova et al., 1997) at the very beginning of translation initiation, by displacing the translation initiation factor eIF4G from mRNA (Nekrasov et al., 2003). Sequence specific RNA binding of YB-1 has also been shown in vivo (Bouvet et al., 1995).

YB-1 interacts with glutathione peroxidase transcripts at the seleno-cysteine insertion sequence located 3' to alternatively read UGA codons (Shen et al., 1998). YB-1 is also one of the first detected RNA binding proteins affecting CD44 alternative splicing (Stickeler et al., 2001). It interacts with RNA binding protein hnRNPK (Funke et al., 
1996; Shnyreva et al., 2000) and is also speculated to bind to splicing factor 1 (SF1) (Stickeler et al., 2001). It is involved in translation regulation of iron-responsive elementbinding protein (IRP-BP/IRP2) (Ashizuka et al., 2002). YB-1 along with another RNA binding protein, nucleolin, is required for IL2 mRNA stabilization induced by T-cell activation signals (Chen et al., 2000). It is also involved in translational regulation of Cyclin D1, c-jun, mertk and PDGFR- $\beta$ mRNA, by the PI3K-AKT pathway (Evdokimova et al., 2006). It is shown to be controlling transforming growth factor beta-1 (TGF- $\beta 1$ ) translation in proximal tubular cells (Fraser et al., 2007).

In addition to being responsible for translational regulation of many other eukaryotic genes, YB-1 is involved in autoregulation of its mRNA (Skabkina et al., 2005), by binding a regulatory element within the $3^{\prime}$ untranslated region (UTR) of YB-1 mRNA. YB-1 strongly and specifically inhibits its own synthesis at the stage of initiation, with accumulation of its mRNA in the form of free mRNPs. Poly (A) binding protein (PABP) competes with YB-1 for binding to the YB-1 mRNA regulatory element and restores the translational activity of YB-1 mRNA, in a Poly (A) tail independent manner (Skabkina et al., 2003). Some studies also show that YB-1 protein autoregulates its own translation by binding to the 5'-UTR of its mRNA (Fukuda et al., 2004).

\subsubsection{DNA repair}

Numerous studies implicate YB-1 in DNA repair. YB-1, which is expressed ubiquitously in human tissues at basal levels, is over expressed in human cancer cell lines that are resistant to cisplatin, where it binds to cisplatin modified DNA (Ohga et al., 1996). It has also been shown to bind preferentially to apurinic DNA or single stranded DNA, but not to UV-irradiated DNA, suggesting that it binds preferentially to only structurally altered DNA (Hasegawa et al., 1991). YB-1 is shown to interact with proliferating cell nuclear antigen, PCNA and possess a $3^{\prime} \rightarrow 5^{\prime}$ exonuclease activity (Ise et al., 1999). A yeast two-hybrid system for base excision repair enzymes identified YB-1 interaction with human endonuclease III enzyme and hNth1 (Marenstein et al., 2001). YB-1 is also shown to be capable of promoting rapid annealing of complementary nucleic acid strands (Skabkin et al., 2001). It was shown to actively promote strand separation of duplex DNA containing either mismatches or cisplatin modifications independently of the nucleotide sequence and also shown to associate directly or indirectly with DNA repair proteins MSH2, Ku80 and WRN in vitro (Gaudreault et al., 2004). The preferential binding to structurally altered DNA, its stress inducible nuclear localization and its ubiquitous distribution within all tissues of higher organisms is indicative that YB-1 plays an important role in DNA repair mechanism.

\subsubsection{Drug resistance}

Multiple studies underscore the role of YB-1 in drug resistance. Overexpression of YB-1 in cancer cells was shown to result in their resistance to etoposide and doxorubicin (Bargou et al., 1997) indicating that YB-1 might be one of the factors responsible for 
drug resistance. Disruption of YB-1 by the antisense approach resulted in sensitizing the cells to drugs (Ohga et al., 1996 and 1998), implicating YB-1 specifically for this function. YB-1 was shown to up regulate the expression of mdr-1, mrp-1 and mvp genes (Stein et al., 2001 and 2005), which are involved in drug resistance. Expression of mdr-1 gene was shown to result in production of p-glycoprotein, an ABC transporter, responsible for efflux of chemotherapeutic compounds from cancer cells, thereby rendering them drug resistant (Choudhuri et al., 2006). Disruption of one allele of YB-1 gene in mouse embryonic stem cells resulted in the mutated cells showing defects in drug resistance and increased sensitivity to cisplatin and mitomycin C (Shibahara et al., 2004).

\subsubsection{Stress response}

YB-1 is a nucleocytoplasmic shuttling protein, involved in many DNA and RNA dependent events. Therefore distribution of YB-1 between nuclear and cytoplasmic compartments must be stringently controlled. YB-1 is an integral part of the general stress response signaling pathway required for protecting cells from a variety of stresses. Nuclear translocation of YB-1 has been shown as a stress response to various extracellular insults, including adenovirus infection (Holm et al., 2002), UV irradiation (Koike et al., 1997), hyperthermia (Stein et al., 2001), anti cancer drugs (Uchiumi et al., 1993) or association with certain proteins such as tumor suppressor p53 and splicing factor SRp30c (Raffetseder et al., 2002). It was shown that YB- $1^{-/-}$embryos demonstrate reduced abilities to respond to oxidative, genotoxic and oncogene induced stresses (Lu et al., 2005).

\subsubsection{Cell proliferation}

YB-1 is shown to be involved in cell proliferation both indirectly and directly. Several studies show that YB-1 protein levels are upregulated in actively proliferating cells like embryonic and cancer cells as compared to quiescent or non-proliferating cells (Oda et al., 1998; Shibao et al., 1999; Kamura et al., 1999; Hipfel et al. 2000; Janz et al., 2002) and regenerating liver tissue following chemical induced damage (Grant et al., 1993) or hepatectomy (Ito et al., 1994). YB-1 expression is seen throughout development and its expression levels correlate with the cell proliferative states (Lu et al., 2005). YB-1 is involved in activation of many genes implicated in cell proliferation like thymidine kinase (Ladomery et al., 1995), growth hormone receptor genes (Swamynathan et al., 1998), proliferating cell nuclear antigen (PCNA) (Ise et al., 1999; Gu et al., 2001), Cyclin A and B1 genes (Royer et al., 2003), DNA Polymerase $\alpha$ (En-Nia et al., 2005), PDGF- $\beta$ in mesangioproliferative glomerular disease (Mertens et al., 2005) and epidermal growth factor receptor (Dunn et al., 2007). YB-1 is probably responsible for viral replication of DNA (Holm et al., 2002). It is reported to be a repressor of cell death associated gene, fas (Lasham et al., 2000).

YB-1 is overexpressed in malignant tissues of breast cancer patients (Bargou et al., 1997), non-small cell lung cancer (Shibahara et al., 2001; Gu et al., 2001), ovarian serous 
adenocarcinomas (Kamura et al., 1999), human oseteosarcomas (Oda et al., 1998), colorectal carcinomas (Shibao et al., 1999) synovial sarcoma (Oda et al., 1999) and malignant melanomas (Hipfel et al., 2000). Diffuse expression of YB-1 is seen in dysplasia-associated lesions or masses of ulcerative colitis, both of which are associated with a high risk of cancer (Fogt et al., 2001). Presence of YB-1 can identify high-risk patients in both the presence and absence of post chemotherapy and independently known tumor biologic factors (Janz et al., 2002). It was also shown that YB-1 promotes breast cancer through induction of chromosomal instability that emerges from mitotic failure and centrosome amplification (Bergmann et al., 2005).

YB-1 has also been shown to be involved in cell proliferation by disruption of its alleles resulting in a reduction in growth. Disruption of both alleles of chicken YB-1 gene at the cold shock domain in DT-40 cells caused a slow growth phenotype in YB-1 ${ }^{-/}$cells (Matsumoto et al., 2005). A homozygous null mice with a true null mutation of YB-1 exhibited severe growth retardation and progressive mortality of $\mathrm{YB}-1^{-/-}$embryos after E13.5 (Lu et al., 2005). YB1 ${ }^{-/-}$fibroblasts from a double knock out mouse showed greatly reduced cell proliferation and altered cell morphology, demonstrating the critical role played by YB-1 in cell proliferation (Uchiumi et al., 2006). Downregulation of YB-1 by shRNA was shown to result in significant reduction in rate of proliferation and increased rate of apoptotic cell death (Schittek et al., 2007).

\subsection{Previous Research}

\subsubsection{Introduction}

The precise modification of chromosomal sequences by targeted integration of artificial constructs provides a powerful approach to determine the function of genes and regulatory elements within a living cell. In the last few years, many studies have been done to study the role of YB-1 in cell proliferation, by knocking down one or both alleles of the gene in different systems. One of the very first of such studies was reported by our laboratory in 2002 (Swamynathan et al., 2002). This study involved the disruption of one allele of chicken YB-1 gene in DT-40 cells and investigation of the defects seen in the heterozygous mutants.

DT-40 is a chicken pre-B lymphocyte cell line, which allows efficient gene disruption due to its high homologous recombination activity (Buerstedde et al., 2002). It has been proven to be a cell line popular in the study of cell autonomous (Nakayama et al., 2001; Um et al., 2001; Takata et al., 2001), targeted disruption of a number of genes (Takeda et al., 1992; Wang et al., 1996; Takami et al., 1997) and B-cell specific

processes (Bezzubova et al., 1997; Kurosaki et al., 1997), due to high ratios of targeted to random integration of transfected constructs (Buerstedde et al., 1991). 


\subsubsection{Strategy used for disruption of one allele of Chk-Yb-1b gene}

A neomycin resistance gene, NeoR, under the control of SV40 promoter/enhancer was introduced into the Chk-YB-1b gene in DT-40 cells, in order to replace a 400 bp StyI fragment overlapping the first exon-intron region, by homozygous recombination (Fig. 1.3). This resulted in a disruption of one of the alleles, which was confirmed by Southern blotting and PCR amplification experiments.

Due to this disruption in one allele, normal splicing and formation of full length mRNA is expected of only one allele, whereas the disrupted allele is expected to undergo defective splicing, leading to the final formation of one copy of normal mRNA and one copy of disrupted mRNA and their respective proteins (Fig. 1.4).

\subsubsection{Defects seen in the heterozygous mutant DT-40 cells}

Numerous defects were detected in the heterozygous mutant DT40 cells. One of the most interesting defects seen was a defective cell cycle. When DNA content of the wild type and mutant DT-40 cells was analyzed by FACS analysis, a large proportion of the mutant cells showed 4n DNA in contrast to the wild type cells with 2n DNA (Fig. $1.5 \mathrm{~A}$ ). This suggests that the cell cycle defect in mutant cells is past the DNA synthesis or S-phase of cell cycle. Approximately $25-30 \%$ of the cells also showed about $6 \mathrm{n}$ or greater content of DNA, indicating re-replication of DNA without cell division. This result strongly suggests that the defect in cell cycle is very likely at the G2/M phase.

DT40YB- $1 \mathrm{~b}^{+/-}$cells were also found to grow slowly, with a doubling time of more than 36-40 hrs. when compared to the 12-14 hrs. for wild type cells (Fig. 1.5B). This indicates that disruption of one allele of YB-1 has a marked effect on cell proliferation. Some morphological abnormalities were also detected in the heterozygous mutants. In contrast to wild type DT-40 cells, which were small and well rounded with a smooth surface, the mutant cells showed a larger (1.5-2 times), irregular appearance with hairy projections from the rough cell surface (Fig. 1.5C).

Heterozygous mutant DT-40 cells also showed some evidences of apoptosis. Condensed chromatin with distinct sub-nuclear fragments, which is a hallmark of apoptotic cells, was detected in case of DT40YB- $1 \mathrm{~b}^{+/-}$cells, by cellular staining with Hoechst 33258 vital dye (Fig. 1.5D), whereas the nuclei of wild type cells stained uniformly. Fragmentation of DNA in the form of multimers of nucleosome length DNA was also seen in the mutant cells, but not in wild type cells (Fig. 1.5E), clearly indicating that the mutant cells underwent apoptosis.

DNA microanalysis of 120 cell cycle related genes in DT40YB- $1 \mathrm{~b}^{+/-}$cells showed that there was a downregulation of genes for cyclin dependent kinase inhibitor $\mathrm{p} 21$, replication factor C 40 protein, MM-1, MAPKK1, GADD153 and C-jun N-terminal kinase $3 \alpha 2$. Enhanced expression of Ser/Thr protein kinase, replication factor C 37, 

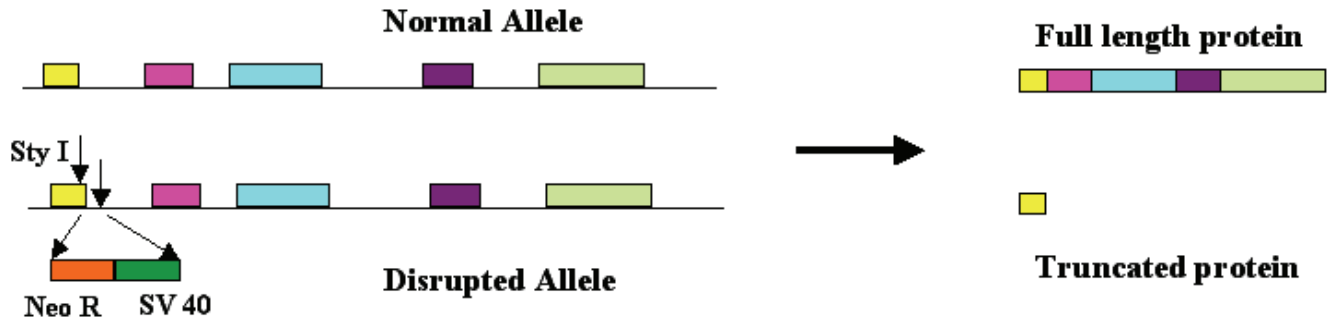

Truncated protein

Fig. 1.3 Schematic representation of disruption of one allele of YB-1 gene The targeted disruption of one allele of Chk-YB-1b gene at its N-terminal Sty I site resulted in one copy of wild type YB-1 protein and a potentially truncated protein spanning the YB-1 N-terminus up to the Sty I site.
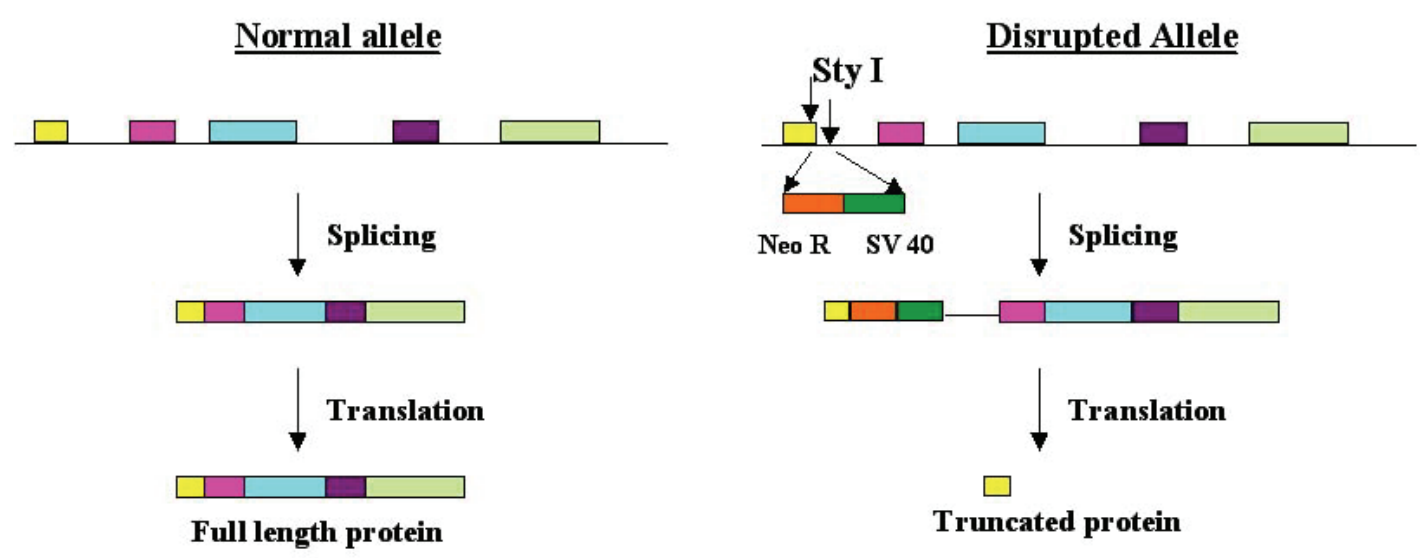

Fig. 1.4 Schematic representation of splicing of the two alleles of Chk-YB-1 gene after the disruption of one allele

After the targeted disruption of one allele of chicken YB-1 gene, normal splicing and formation of full length mRNA is expected of only the wild type allele, whereas the disrupted allele is expected to undergo defective splicing, leading to the final formation of one copy of normal mRNA and one copy of disrupted mRNA and their respective proteins. 
Fig. 1.5 Defects seen in the heterozygous mutant DT-40 cells

A) FACS analysis of wild type and mutant DT-40 cells stained with propidium iodide shows increased genomic DNA content in the heterozygous mutant DT-40 cells.

B) Growth curve of wild type and mutant DT-40 cells shows that the doubling time of the mutant cells was 3-4 times higher than the wild type cells.

C) Morphological defects were seen in the mutant DT-40 cells, which were large, irregular and rough surfaced, in comparison with the wild type cells, which are small and spherical with smooth edges.

D) Evidence in the form of chromatin condensation is seen in the mutant DT-40 cells stained with the vital dye, Hoechst 33258, as compared to the wild type cells.

E) DNA fragmentation (fragments of multimers of unit nucleosomal size DNA) was seen only in case of the mutant DT-40 cell DNA, but not in the wild type cell DNA.

F) Microarray analysis of 120 genes in the mutant and wild type cells revealed that some genes are upregulated or downregulated in the mutant cells when compared to the wild type cells. Genes showing significant levels of differences are shown in this figure. (Reprinted with permission. Swamynathan, S.K, Varma, B.R., Weber, K.T., and Guntaka, R.V. (2002) Targeted disruption of one allele of the Y-box protein gene, ChkYB-1b, in DT40 cells results in major defects in cell cycle. Biochem Biophys Res Commun. 296, 451-457.) 
A

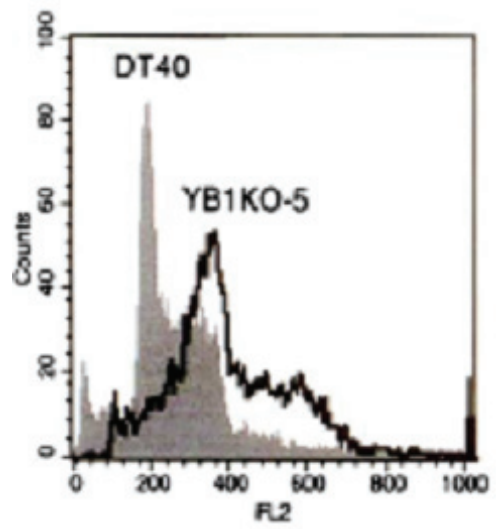

C

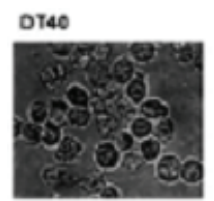

Y81K07
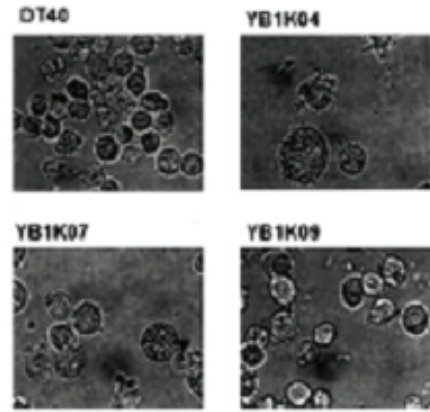

reikos

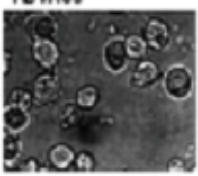

B

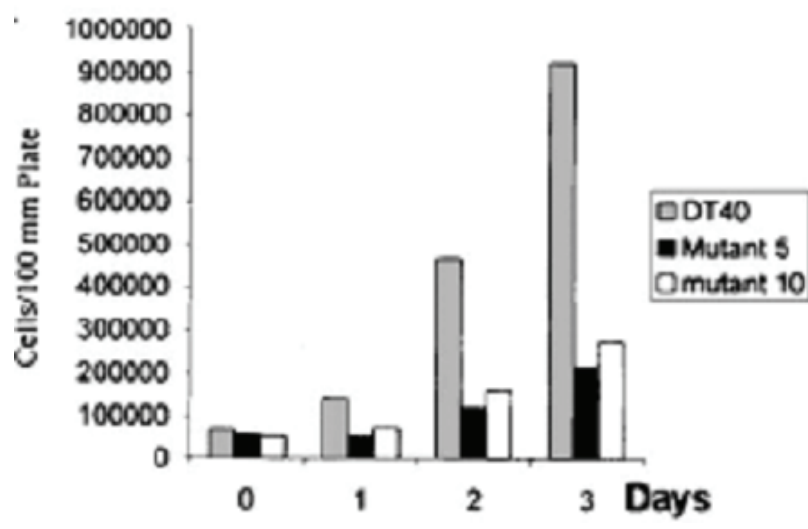

D
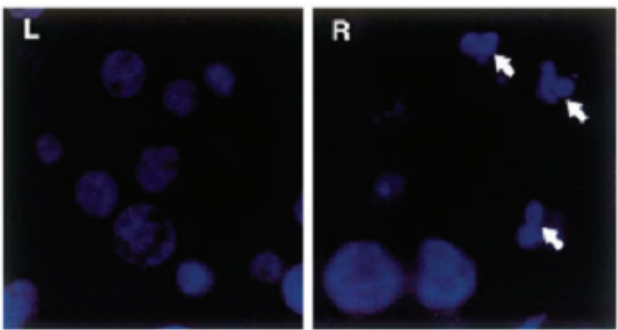

F
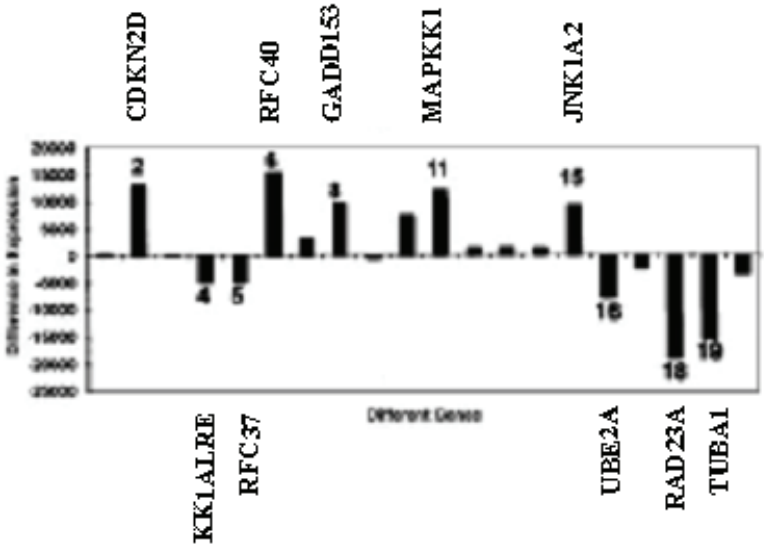
ubiquitin conjugated enzyme E2, UV excision repair protein, RAD23A and tubulin $\alpha 1-$ subunit genes was seen (Fig. 1.5F).

\subsection{Hypothesis}

Equal amounts of YB-1 full length mRNA and protein were seen to be present in both the wild type and heterozygous mutant DT-40 cells. Therefore the abnormalities seen cannot be attributed to the decrease of full length YB-1 protein in mutant cells. Our hypothesis is that altered phenotype of mutant DT-40 cells could be due to a dominant negative effect, exerted by a potential truncated protein spanning the N-terminus of YB-1 up to the Sty I site at the amino acid 77, encoded by the disrupted allele. If so, introduction of the exogenous fusion protein carrying the N-terminal sequence of YB-1 should mimic the phenotypic changes.

DT40YB- $1 \mathrm{~b}^{+/-}$cells also showed a change in expression of a few cell cycle related genes, including the cyclin dependent kinase inhibitor, p21. We propose the possibility of YB-1 interacting with some of these cell cycle proteins at a protein level, especially at the G2/M phase, since the defects seen in the mutant DT-40 cells were most likely at the $\mathrm{G} 2 / \mathrm{M}$ phase of cell cycle.

\subsection{Significance of this study}

In the past couple of years, there has been a rapid progress in understanding the function of YB-1. However current knowledge about the molecular mechanisms underlying the role of various domains of YB-1 in cell proliferation is limited. The first part of this project involves study of the effects of introduction of YB-1 N-terminal protein into mammalian cells. The experiments are designed to investigate if the introduction of YB-1 N-terminal protein results in an effect on the cell cycle and cell survival of the mammalian cells. Our results demonstrate that the YB-1 amino end protein internalization results in cell cycle arrest at the $\mathrm{G} 2 / \mathrm{M}$ phase and apoptosis. In order to understand the integrated functions of YB-1, it is essential to study all its molecular interactions with the other proteins. The second part of this project is designed to investigate the interaction of YB-1 with the cell cycle protein, cyclin D1. The results indicated that full length YB-1 interacts and co-localizes with cyclin D1 at the G2/M phase of cell cycle predominantly in the cytoplasm of mammalian cells. The YB-1 $\mathrm{N}$-terminal protein is also able to interact with the cyclin D1 in the cytoplasm of nocodazole-blocked cells.

Overall this analysis of YB-1 has provided an important insight into its role in cell proliferation and in oncogenesis. Furthermore, understanding this YB-1 interaction with

cyclin D1 may offer novel strategies for the development of unique anti-cancer drugs. 


\section{Chapter 2. YB-1 Amino Terminal Domain Plays a Major Role in Cell Proliferation and Apoptosis}

\subsection{Introduction}

YB-1 is a multifunctional protein, involved in a multitude of important cellular functions, including cell proliferation. Numerous studies demonstrate a role of YB-1 in cell proliferation. YB-1 protein levels are upregulated in actively proliferating cells like embryonic and cancer cells as compared to quiescent or non-proliferating cells (Oda et al., 1998; Shibao et al., 1999; Kamura et al., 1999; Hipfel et al. 2000; Janz et al., 2002) and regenerating liver tissue following chemical induced damage (Grant et al., 1993) or hepatectomy (Ito et al., 1994). YB-1 expression is seen throughout development and its expression levels correlate with the cell proliferative states (Lu et al., 2005), for example, expression of YB-1 in multiple myeloma cells is associated with a highly proliferative phenotype (Chatterjee et al., 2007). YB-1 is also involved in activation of many genes implicated in cell proliferation like thymidine kinase (Ladomery et al., 1995), growth hormone receptor genes (Swamynathan et al., 1998), proliferating cell nuclear antigen (PCNA) (Ise et al., 1999; Gu et al., 2001), Cyclin A and B1 genes (Royer et al., 2003), DNA Polymerase $\alpha$ (En-Nia et al., 2005), PDGF- $\beta$ in mesangioproliferative glomerular disease (Mertens et al., 2005) and epidermal growth factor receptor (Dunn et al., 2007).

In order to gain a better insight into the role of YB-1 in cell proliferation, studies have been done by knocking out one or both alleles of YB-1. One of the very first of a series of such studies published by our laboratory showed that a targeted disruption of one allele of chicken YB-1 gene in DT40 cells at the amino terminal domain, resulted in major cell cycle defects like increased doubling time, increased genomic DNA content, increased cell size and apoptosis in a fraction of the cell population (Swamynathan et al., 2002). In contrast, targeted disruption of one allele of YB-1 gene at the carboxy terminal domain in mouse embryonic stem cells resulted in no apparent growth defects in the mutated cells (Shibahara et al., 2004). However the mutated cells exhibited defects in drug resistance and sensitivity to cisplatin and mitomycin $\mathrm{C}$. Another study involving the disruption of both alleles of chicken YB-1 gene at the cold shock domain in DT-40 cells, demonstrated a slow growth phenotype in YB-1 ${ }^{-/}$cells but no apparent growth defect in heterozygous mutants with one YB-1 disrupted allele (Matsumoto et al., 2005). The mutant cells showed major translational defects. A comparison of these studies (Fig. 2.1) shows the disruption of each domain of YB-1, either on one or both alleles of the gene, resulting in defects in different functions, suggests that each domain is involved in some specific cellular functions.

A non-redundant role of YB-1 in cell proliferation and growth was suggested by creating homozygous mice with a true null mutation in the YB-1 gene (Lu et al., 2005). This study showed that after E13.5, YB- $1^{-/-}$embryos exhibited severe growth retardation and progressive mortality. The $\mathrm{YB}-1^{-/-}$fibroblasts demonstrated reduced abilities to respond to oxidative, genotoxic and oncogene induced stresses. A definitive role for 


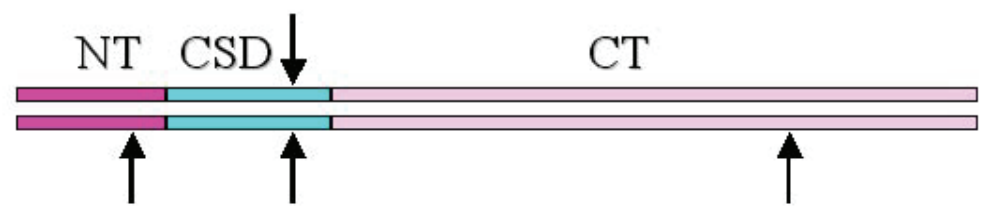

NT

(Swamynathan et. al., 2002)

\section{Growth defects}

NT + CSD

Translational defects

(Matsumoto et. al., 2005)

$\mathrm{NT}+\mathrm{CSD}+\mathrm{CT}$

(Shibahara et. al., 2004)

DNA repair and drug resistance defects

\section{Full Length YB-1}

Fig. 2.1 Comparison of disruption made in the YB-1 domains by various investigators and their corresponding defective functions

Arrows indicate a representation of disruptions made in each of the three domains of YB-1. As seen from the three studies, disruption in each of the domains results in defects in different functions of YB-1, suggesting a possibility of each of the YB-1 domains being responsible for those particular functions. 
YB-1 in cell proliferation has been proven by double knock out mice by disruption of both alleles of YB-1 leading to embryonic lethality (Uchiumi et al., 2006). Further studies with $\mathrm{YB1}^{-/-}$fibroblasts showed greatly reduced cell proliferation and altered cell morphology, demonstrating the critical role played by YB-1 in cell proliferation. Latest studies showed that downregulation of YB-1 by shRNA results in significant reduction in rate of proliferation and increased rate of apoptotic cell death (Schittek et al., 2007).

Indepth analysis of the phenotypic abnormalities seen in the heterozygous mutant DT-40 cells from our study suggested that the N-terminal domain of YB-1 might be involved in the regulation of cell proliferation by YB-1. We ruled out the possibility of attributing the defects to a decrease in wild type YB-1 mRNA or protein, since no reduction in either of their levels was detected. This was most likely due to the tetraploid phenotype of the DT-40 YB-1 ${ }^{+/}$cells. We then speculated that a possible reason for the altered phenotype could be the dominant negative effect exerted by a potentially truncated protein encoded by the disrupted allele. Owing to antibody restrictions for a chicken cell system, detection of a clear band of truncated protein was difficult. Therefore, testing our hypothesis warranted the use of another cell system and an exogenous source of the potential truncated protein. If our hypothesis is right, introduction of the exogenous fusion protein carrying the N-terminal sequence of YB-1 should mimic the phenotypic changes.

The plasma membrane constitutes a lipid bilayer inserted with proteins and glycoproteins. The hydrophobic nature of these lipids poses a barrier for a vast majority of peptides and proteins from crossing the membrane. Therefore, to introduce the YB-1 $\mathrm{N}$-terminal proteins into the cells an appropriate mode of delivery was required.

Numerous vehicles are available for delivering proteins into cells, including the protein transduction domains (PTDs), also known as cell penetrating peptides (CPPs), which are capable of transporting biological cargo from the surrounding medium into the cells. Previous literature shows that antennapedia and TAT conjugates are the most extensively used delivery systems. For our purpose, we were looking for a peptide delivery system, which could be used at optimal concentrations for longer time periods, with reasonably low cytotoxicity and yet providing efficient and rapid uptake of the bound peptide to be delivered into cells. It has been shown previously that in terms of uptake and toxicity, antennapedia proves to be the most optimal cell penetrating peptide for peptide delivery in vitro as compared to the other peptides (Jones et al., 2005). A 16-amino acid long polypeptide corresponding to the third helix of antennapedia homeodomain (RQIKIWFQNRRMKWKK) is the minimal sequence required for successful translocation across the cell membrane (Derossi et al., 1994 and1996). Therefore to deliver the YB-1 truncated proteins into cells we utilized the antennapedia homeodomain.

We constructed three different YB-1 fusion protein clones including i) APGFP, the control fusion protein without any YB-1 sequence, ii) APYB77GFP, the 77 amino acid long full length YB-1 N-terminal sequence containing clone, iii) APYB36GFP, the 36 amino acid long peptide with the internal deletion $\Delta 12-52$ (APYB36 $\Delta 12-52 \mathrm{GFP}$ represented as APYB36GFP) containing clone and iv) APYB26GFP, the 26 amino acid long proline rich YB-1 N-terminal sequence containing clone. The APYB26GFP fusion 
protein was constructed keeping in mind the importance of proline rich domains in various cellular functions. Proline rich domains are important for signal transduction (Vidal et al., 2001). They are needed for proper protein folding (Kusano et al., 2001) and important for growth hormone receptor signal transduction (Goujon et al., 1994). YB-1 $\mathrm{N}$-terminus has a stretch of 26 amino acids, which are extremely rich in alanines and prolines. To test our hypothesis implicating the YB-1 N-terminus for its role in cell proliferation, it is imperative to study the significance of just the proline rich sequence.

The YB-1 N-terminal fusion peptide sequences were expressed as fusion proteins with sequences encoding the antennapedia for internalization and GFP for visualization inside cells. The rat hepatoma cell system was used for testing of our hypothesis. The objective of this study was to investigate if introduction of these N-terminal peptides, using the antennapedia mimics the defects seen in the heterozygous mutant DT-40 cells from the previous study and thereby test our hypothesis.

\subsection{Materials and methods}

\subsubsection{Antibodies and reagents}

All the chemical reagents, unless otherwise specified were purchased from SigmaAldrich (St. Louis, MO). The Minimum essential medium (cat \# 11095-080), Dulbecco's modified medium (cat \# 1965-092), Penicillin-Streptomycin (cat \# 15140-148) and Trypsin-EDTA (cat \# 25300) was obtained from Invitrogen-Gibco (Carlsbad, CA). The YB-1 polyclonal antibody $(10 \mathrm{mg} / \mathrm{ml})$ was generated for our lab by Sigma. The antibody against GFP (cat \# A11122, $2 \mathrm{mg} / \mathrm{ml}$ ) and the fluorescent-labeled secondary antibodies (cat \#s A21429 and A11029, 2mg/ml) were from Invitrogen-Molecular probes (Carlsbad, CA). The chemiluminescence western blot developing solution kit (cat \# 34080) and the BCA protein assay kit (cat \# 23225) was from Pierce (Rockford, IL). The Vectashield Mounting Medium containing DAPI (cat \# H-1200) was from Vector laboratories (Burlingame, CA). The protein A Sepharose beads (cat \# 17-0780-01) were from

Amersham Biosciences (Piscataway, NJ). The Aposcreen ${ }^{\text {TM }}$ Annexin V-FITC kit (cat \# 10010-02) was from Southern Biotechnology Associates Inc. (Birmingham, AL).

\subsubsection{Cloning and expression of APGFP-fusion proteins}

The strategy used for cloning is shown in Fig. 2.2. The three amino-terminal YB-1 sequences used for cloning are shown in Fig. 2.3. pUC966 vector modified in our lab was used as a backbone for all sequences. The 26 amino acid alanine-proline rich YB-1 $\mathrm{N}$-terminal sequence was cloned at the EcoR1 site. By varying our template and PCR conditions the 36 amino acid long truncated version of YB-1 N-terminus with an internal deletion of $\Delta 12-52$ and the 77 amino acid long full length YB-1 N-terminus were cloned at the Bam H1 site of pUC966 vector. All sequences were cloned inframe with an upstream coding sequence for the 16 amino acid long antennapedia homeodomain and 

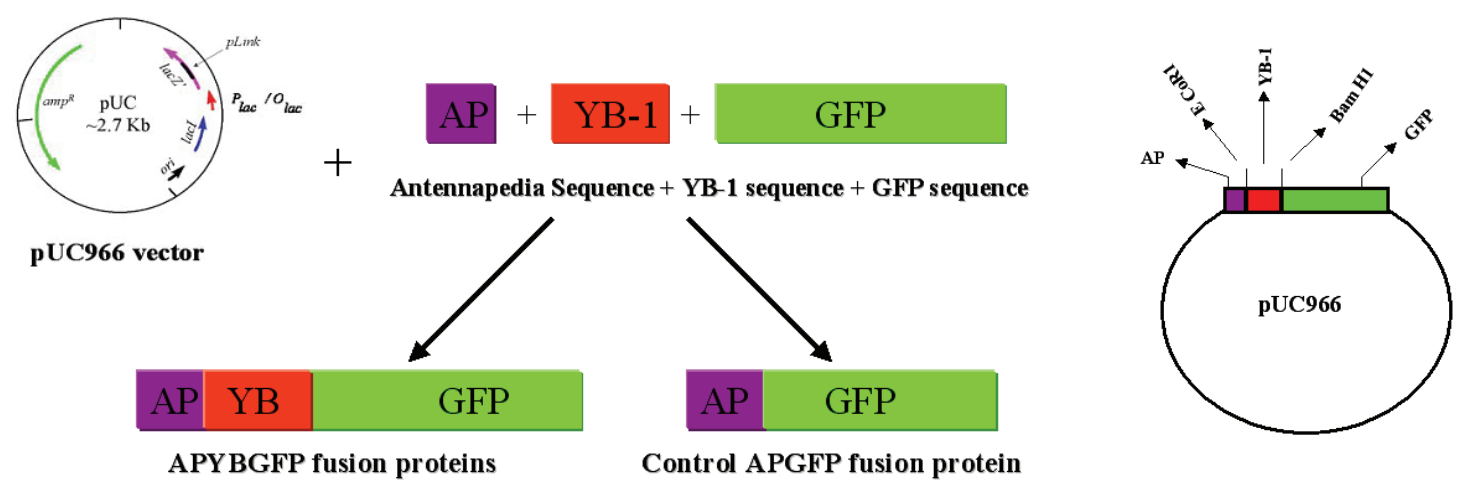

Fig. 2.2 Strategy of cloning the APGFP fusion proteins

The APGFP fusion proteins were cloned in pUC966 vector. The YB-1 N-terminal sequences were flanked by the antennapedia sequence on its $3^{\prime}$ end and the GFP sequence on its $5^{\prime}$ end.

$$
\begin{aligned}
& \underline{\text { YB }-77} \\
& 1 \\
& \text { N-M S S E A T Q QPPA APPA APALS A ADT KPG T T GS G A G S G G P G G L } \\
& \text { T S A A P A G G D K K VI A T K V L G T V K W F N V R N G Y G F I N R N D T K D - } \underline{\text { C }} \\
& \underline{\text { YB }-26}
\end{aligned}
$$

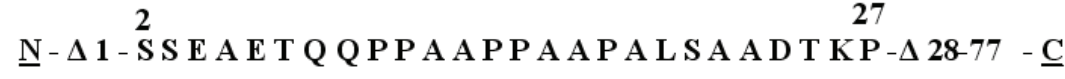

$$
\begin{aligned}
& \underline{\text { YB }-36} \\
& 1 \quad 11 \quad 53 \quad 77 \\
& \underline{N} \text {-M S S E A E T Q Q P P- } \Delta \text { 12-52 - KVLG T V KW F NVRNG Y G FINRNDT KD- } \underline{\text { C }}
\end{aligned}
$$

\section{Fig. 2.3 YB-1 N-terminal amino acid sequences used for cloning}

For each construct the YB-1 N-terminal amino acids and their positions in YB-1 protein are shown. APYB77GFP contains the initial 77 amino acids from YB-1 N-terminus.

APYB26GFP sequence contained 26 amino acids proline alanine rich sequence from the YB-1 N-terminus. APYB36GFP contains 36 amino acids from the initial 77 amino acids of the YB-1 N-terminus with an internal deletion of amino acids in position 12 to 52 . 
downstream coding sequence for GFP. E. Coli Top 10 competent cells were used for transformation and plasmid isolation. The DNA sequences and insert orientation were confirmed by DNA sequencing (University of Tennessee, Molecular Resource Center). The three clones APYB26GFP, APYB36GFP and APYB77GFP and the control clone APGFP, which lacked YB-1 sequence, were then transformed into E.Coli BL21DE3 cells. Protein expression was induced by $1 \mathrm{mM}$ IPTG in a culture of exponentially growing cells. Overnight-induced cultures were harvested by centrifugation at $5000 \mathrm{~g}$ for $15 \mathrm{~min}$. at $4^{\circ} \mathrm{C}$. The pellets were stored at $-80^{\circ} \mathrm{C}$ or processed for purification of fusion proteins.

\subsubsection{Purification of proteins}

Cell lysis and isolation of inclusion bodies were done as described previously (Marston et al., 1984; Marston 1987). In short, each gram of pellet was resuspended in 3 $\mathrm{ml}$. lysis buffer (50 mM Tris-Cl (pH 8.0), 1mM EDTA and $100 \mathrm{mM} \mathrm{NaCl}) .8 \mu 1$ of 50 $\mathrm{mM}$ PMSF and $80 \mu 1$ of $10 \mathrm{mg} / \mathrm{ml}$ lysozyme was added to it and left on ice for $20 \mathrm{~min}$. with occasional stirring. $4 \mathrm{mg}$ of deoxycholic acid per gram of pellet was added to this mixture while stirring continuously. It was stirred at $37^{\circ} \mathrm{C}$ until the lysate became viscous. $20 \mu 1$ of $1 \mathrm{mg} / \mathrm{ml}$ DNAse I was added and the lysate was kept at room temperature until it was no longer viscous (about 30-45 min.). It was then centrifuged at $12000 \mathrm{~g}$ for $15 \mathrm{~min}$. at $4^{\circ} \mathrm{C}$. The supernatant was frozen and stored at $-80^{\circ} \mathrm{C}$. The pellet was resuspended in 9 volumes of lysis buffer with the same composition as mentioned previously containing $0.5 \%$ Triton X-100 and 10mM EDTA $(\mathrm{pH} 8.0)$ and kept at room temperature for $5 \mathrm{~min}$. The lysate was centrifuged as mentioned previously and the supernatant was stored separately. Equivalent quantities of the supernatants and pellet were analyzed on a $12 \%$ SDS-PAGE gel with coomassie blue staining. We detected most of our proteins in inclusion bodies.

For solubilization of inclusion bodies, the pellets were resuspended in 10 volumes solubilization buffer ( $8 \mathrm{M}$ Urea and $10 \mathrm{mM} \beta$-Mercaptoethanol) and incubated on a nutator for $5 \mathrm{hrs}$. at $4^{\circ} \mathrm{C}$, followed by centrifugation at $11000 \mathrm{~g}$. The supernatant was frozen at $-80^{\circ} \mathrm{C}$ and the pellet was again resuspended in the solubilization buffer for similar processing. The fusion proteins were further purified from the supernatants using DEAE Sephacel ion-exchange chromatography at $4{ }^{\circ} \mathrm{C}$ as described previously (Crawford et al., 2004). The expected fusion proteins were detected in fractions eluted with $0.3 \mathrm{M}$ $\mathrm{NaCl}$. These fractions were dialyzed overnight at $4{ }^{\circ} \mathrm{C}$ with 4 changes of buffer $(50 \mathrm{mM}$ Tris- $\mathrm{Cl}$ (pH 8.0), $100 \mathrm{mM} \mathrm{NaCl}$ and 5\% glycerol). Purity of the proteins was determined by running triplicates of the protein fractions on a $15 \%$ SDS-PAGE gel for coomassie staining, silver staining and western blotting using an antibody against the GFP. All the proteins were purified to more than $70 \%$ as assessed by polyacrylamide gel electrophoresis, followed by coomassie blue staining as shown in Fig. 2.4. The organization of these constructs and their respective sizes on a western blot are shown in Fig. 2.5. Western blot analysis revealed only the fusion proteins of expected sizes. Protein concentration was checked using the BCA protein assay kit. 


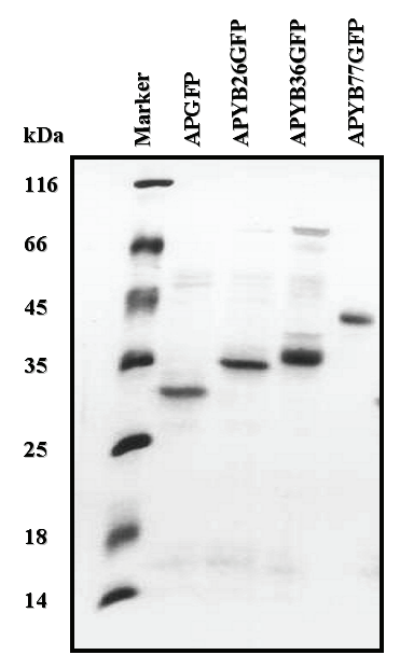

\section{Fig. 2.4 Purified fusion proteins on a SDS-PAGE gel}

The APGFP fusion proteins were purified by DEAE-sephacel chromatography, dialyzed, quantitated and equivalent amounts analyzed for purity by polyacrylamide gel electrophoresis and coomassie blue staining. The molecular weights are shown towards the left of the gel picture. The proteins got are about $80 \%$ pure.

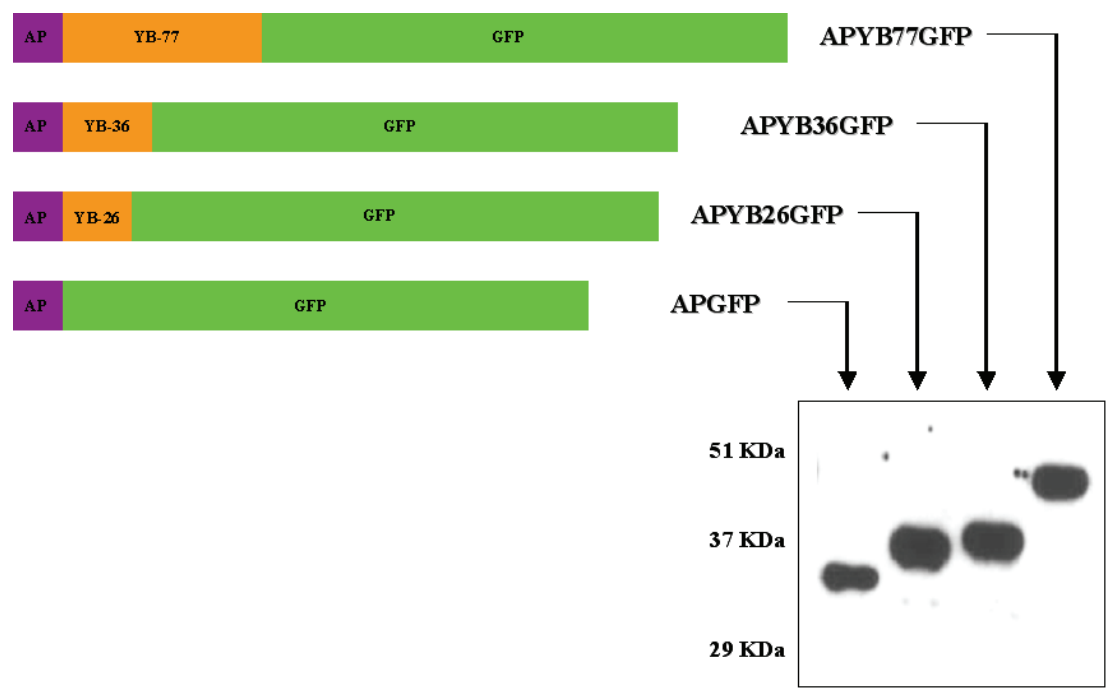

Fig. 2.5 Schematic representation of the four clones and their sizes on a SDS-PAGE Western blot

The YB-1 sequences were cloned with an upstream antennapedia and downstream GFP sequence. APGFP clone lacks the YB-1 sequence and is used as a control fusion protein. The organization of the four clones and their respective sizes on a western blot probed with GFP antibody is shown. The molecular weights are mentioned on the left of the gel. 


\subsubsection{Cell culture}

Rat Hepatoma cells (ATCC \# H-411E) were grown in monolayers in Minimum Essential Medium (MEM) supplemented with 10\% FBS, 100U/ml Penicillin and 100 $\mu \mathrm{g} / \mathrm{ml}$ Streptomycin, in a humidified incubator maintained at $37^{\circ} \mathrm{C}$ and $5 \% \mathrm{CO}_{2}: 95 \%$ air. Cells were fed every day and subcultured 1:5 when they became confluent, using $0.05 \%$ trypsin-EDTA. After expansion the cells were frozen in liquid nitrogen in a medium containing a final concentration of $80 \%$ MEM, 10\% FBS and 10\% DMSO. For culture, the frozen cells vial were quickly thawed and added to the prewarmed culture medium mentioned above. The rat aortic smooth muscle (RASM) cells were similarly cultured in Dulbecco's Modified Eagle's Medium (DMEM). For the experiments, cells were incubated with $40 \mu \mathrm{g} / \mathrm{ml}$ of the purified fusion proteins for the mentioned time periods. Where indicated the cells were incubated with $20 \% \mathrm{FBS}$ or U.V. irradiation $\left(40 \mathrm{~J} / \mathrm{m}^{2}\right)$ in the absence of proteins. The U.V. treatment was given in a CAMAG UV cabinet with a UV lamp (100W) set at $366 \mathrm{~nm}$ at a distance of $7 \mathrm{cms}$. from the cells, at room temperature. The total dose provided was $40 \mathrm{~J} / \mathrm{m}^{2}$.

\subsubsection{Immunoblotting}

Cells were washed and lysed with RIPA buffer as described previously (Derossi et al. 1996). Samples were sonicated at Branson 4 setting for 8 cycles of $15 \mathrm{sec}$ each, stored on ice for $30 \mathrm{~min}$. and clarified by high-speed centrifugation at $8000 \mathrm{~g}$. for $5 \mathrm{~min}$. at $4^{\circ} \mathrm{C}$. The supernatant was used as the whole cell extracts. For nuclear and cytoplasmic fractions, the cells were scraped in $100 \mu \mathrm{l}$ ice cold PBS and centrifuged at $500 \mathrm{~g}$ for 5 min. The cell pellet was resuspended in $100 \mu \mathrm{l}$ Buffer A $(50 \mathrm{mM} \mathrm{NaCl}, 10 \mathrm{mM}$ HEPES $\mathrm{pH}-8.0,500 \mathrm{mM}$ sucrose, 1mM EDTA, 0.2\% Triton-X-100, freshly added protease inhibitors $-1 \mu \mathrm{g} / \mathrm{ml}$ leupeptin, $1 \mu \mathrm{g} / \mathrm{ml}$ aprotinin and $1 \mathrm{mM}$ PMSF and $7 \mathrm{mM} \beta$ Mercaptoethanol), vortexed at high speed for $45 \mathrm{sec}$., spun down at $2000 \mathrm{~g}$ for $2 \mathrm{~min}$. at $4^{\circ} \mathrm{C}$ and the supernatant was used as cytoplasmic extract. The pellet was resuspended in $100 \mu \mathrm{l}$ Buffer B (Buffer A with 25\% glycerol and $0.1 \mathrm{mM}$ EDTA) and spun down at $2000 \mathrm{~g}$ for $2 \mathrm{~min}$. at $4^{\circ} \mathrm{C}$. The resulting pellet was resuspended in $50 \mu \mathrm{l} \mathrm{Buffer} \mathrm{C}$ (Buffer $B$ with $350 \mathrm{mM} \mathrm{NaCl}$ ), incubated on ice for $30 \mathrm{~min}$. with intermittent high speed vortexing and spun down at $11000 \mathrm{~g}$ for $15 \mathrm{~min}$ at $4^{\circ} \mathrm{C}$. The resulting supernatant was diluted to $100 \mu \mathrm{l}$ with ice cold PBS was used as nuclear extract. Equivalent amounts of samples were used for immunoblotting on a 15\% SDS-PAGE gel. Rabbit antibody against GFP was used as primary antibody at 1:7500 dilution for overnight incubation at $4^{\circ} \mathrm{C}$ and HRP conjugated Anti-Rabbit IgG was used as secondary antibody at 1:15000 dilution for $2 \mathrm{hrs}$. at room temperature. The blots were developed using chemiluminescence (SuperSignal West Pico Chemiluminescent Substrate) according to the manufacture's protocol. 


\subsubsection{Immunocytochemistry and indirect immunofluorescence}

Cells were rinsed twice with phosphate buffered saline (PBS), fixed for $15 \mathrm{~min}$. with $4 \%$ p-formaldehyde, washed twice with PBS and stored at $4{ }^{\circ} \mathrm{C}$, until all time point samples had been collected. Cells were blocked with 5\% goat serum and 3\% BSA in PBS for $30 \mathrm{~min}$. and rinsed with PBS thrice. Then the cells were incubated with primary antibodies rabbit anti-GFP (1:500) or mouse-anti-YB-1 (1:300) and rabbit anti-cyclin D1 (1:300) for $3 \mathrm{hrs}$. followed by several washes with PBS. Subsequently cells were incubated with secondary antibodies FITC conjugated Anti-Rabbit IgG (1:500) and Texas-Red conjugated Anti-Mouse IgG (1:500) for $1 \mathrm{hr}$. at room temperature in the absence of light. Coverslips were washed several times with PBS and once with water to remove any salts and then left to air-dry for $45 \mathrm{~min}$. in the absence of light. The coverslips were mounted onto glass slides using Vectashield Mounting Medium containing DAPI to counter stain nuclei. The slides were stored at $-20^{\circ} \mathrm{C}$ until analyzed by microscopy.

\subsubsection{Microscopy}

Confocal images were captured using LSM 510 confocal laser-scanning three-color microscope (Carl Zeiss, Jena, Germany). Images were captured using Argon laser at 75\% output and $\mathrm{HeNe} 1$ laser at excitation wavelengths $488 \mathrm{~nm}$ and $543 \mathrm{~nm}$ respectively. Optical slice thickness was set at $0.3 \mu \mathrm{m}$, magnification to $40 \mathrm{x}$ and sections presented were taken approximately at the mid height level of cells. Photomultiplier gain and laser power were identical within each experiment. Apoptotic blebs were seen using Axioplan 2 epifluorescent microscope (Zeiss, Germany). DAPI fluorescence was captured using the $63 \mathrm{X}$ magnification lens of the microscope. Axiovision software was used to capture zvi-stacks of images. Exposure was set using control nuclei and was fixed for each experiment. A minimum of 100 stained cells were scored for each experiment reported. All images were analyzed and adjusted for contrast in Adobe Photoshop 5.0 (San Jose, CA).

\subsubsection{DNA fragmentation}

Exponentially growing cells were incubated with the fusion proteins as mentioned previously for 24, 48 and $72 \mathrm{hrs}$. Analysis of DNA fragmentation was done on these cells as described previously (Swamynathan et al., 2002). In short, treated cells were harvested, suspended in TNE buffer $(1 \mathrm{M} \mathrm{NaCl}, 20 \mathrm{mM}$ Tris-HCl, $\mathrm{pH}$ 7.4, 2mM EDTA and $0.6 \%$ SDS) and incubated overnight on ice. The next day, the cellular debris was spun down at $12000 \mathrm{~g}$ at $4^{\circ} \mathrm{C}$ for $45 \mathrm{~min}$. The supernatant was treated with RNAseA (100 $\mu \mathrm{g} / \mathrm{ml}$ ) for $30 \mathrm{~min}$ at $37^{\circ} \mathrm{C}$. The DNA was phenol chloroform purified and precipitated with ethanol. The DNA pellet was resuspended in TE buffer (Tris-HCl 10mM and EDTA $1 \mathrm{mM}$ ) and an aliquot was used to analyze it on an agarose gel with ethidium bromide staining and photographed under UV illumination (Fotodyne Inc., Hartland, WI). 


\subsubsection{FACS analysis}

For confirmation of successful synchronization and cell cycle analysis, synchronized cells were harvested by trypsinization or scraping on ice and pelleted down at $500 \mathrm{~g}$ for $5 \mathrm{~min}$. The pellet was washed with PBS, cells were fixed with ice cold $70 \%$ ethanol, incubated on ice for $45 \mathrm{~min}$. and pelleted down at $500 \mathrm{~g}$ for $5 \mathrm{~min}$. The pellet was washed with hanks balanced salt solution (HBSS) containing 1\% BSA. The resulting pellet was resuspended in Propidium Iodide buffer (1:1 dilution of $20 \mathrm{ug} / \mathrm{ml}$ of PI in HBSS and $1 \mathrm{mg} / \mathrm{ml} \mathrm{RNAse} \mathrm{A)} \mathrm{and} \mathrm{incubated} \mathrm{for} 30 \mathrm{~min}$. in a $37^{\circ} \mathrm{C}$ water bath. Samples were analyzed using a Becton Dickinson FACS Calibur. The cell cycle analysis software was used to determine the $\%$ of cells in each phase of cell cycle. For analysis of apoptotic cells, Aposcreen ${ }^{\mathrm{TM}}$ Annexin V-FITC kit was used according to the manufacturer's protocol. Cell debris was excluded from the FACS analysis by doing appropriate forward and light scatter gating. Differences in GFP and FITC staining were adjusted by using GFP negative and annexin V FITC positive controls for non specific signals and triplicate experiments with and without annexin V FITC samples and appropriate gating for the experimental samples. Profiles were analyzed and bar graphs were plotted using Microsoft Excel.

\subsubsection{Quantitation of immunoblotting and immunofluorescence data}

All immunoblot images were scanned and exported as TIFF files to Adobe Photoshop software (San Hose, CA) for subsequent analysis. Each image was adjusted to invert the scale. A box was drawn around the biggest band using Marquee tool and histogram analysis was done. The mean was noted down and then the same box was used to drag down to another band to repeat the process. For each band, representative integrated density values were computed after subtracting the background density. For the immunofluorescence data, each stack of confocal images were imported and opened on the LSM image scanner. The histogram analysis was performed on atleast 4 cells from each slice and atleast 4 slices for each experiment and all the slices were analyzed for the same cells. To start with, the outlining tool was used to demarcate the total cell area. The total pixel count was noted down for the whole cell. Then the cell nucleus was demarcated and the nuclear pixel count was noted down. This was done on atleast 4 sections from each cell. The total cell pixels and the total nuclear pixels were added up from all sections and the $\%$ of the nuclear pixels was calculated. This gave the $\%$ of nuclear protein as compared to the total cell protein.

\subsubsection{Data analysis}

For all the FACS analysis experiments, values from three independent experiments were used to draw the graphs on Microsoft Excel. Statistical significance was tested by performing unpaired student's t-tests using the GraphPad Software. Results were considered statistically significant if the $\mathrm{P}$ value of $<0.001$ was reached. However the $\mathrm{P}$ value of each experiment is mentioned separately in the results section. 


\subsection{Results}

\subsubsection{APYBGFP and APGFP fusion proteins get internalized and are stable inside the cells}

Earlier studies from our laboratory indicated that the amino end of YB-1 might exert a role in cell proliferation (Swamynathan et al., 2002, Figs 1.3 and 1.4). Therefore, we expressed a fusion protein containing the 77 amino acids, APYB77GFP in E.coli. Two other fusion proteins containing 36 amino acids with an internal deletion, APYB36GFP and the alanine-proline rich 26 amino acid sequences from YB-1 amino end, APYB26GFP were also expressed. All the constructs contained the fusion facilitating 16 amino acid antennapedia peptide (RQIKIWFQNRRMKWKK) at the amino end and the reporter gene, encoding GFP at the carboxy end of the YB-1 sequence. Three mechanisms have been proposed to explain how Antennapedia bound peptides get transduced across membranes. They are i) inverse micelles are formed and the cargo is captured in an aqueous compartment to move across the membrane (Derossi et al., 1996; Prochiantz, 1996; Binder et al., 2003), ii) the peptide crosses biological membranes in an electroporation like mechanism (Richard et al., 2003) and iii) the peptide relies on the endocytic pathways for it to be delivered into the cells (Richard et al., 2003).

To confirm that our fusion proteins get translocated into and are stable inside the cells for significant time periods, we incubated rat hepatoma cells with $40 \mu \mathrm{g} / \mathrm{ml}$ of each protein for $3 \mathrm{hrs}$. and $18 \mathrm{hrs}$. Western blotting analysis of the whole cell extracts confirmed that the fusion proteins get internalized as early as $3 \mathrm{hrs}$. (Fig. 2.6, top panel) and are stable at the $18 \mathrm{hr}$. time point of incubation (Fig. 2.6, bottom panel). Similar results were obtained with primary cells isolated from rat aortic smooth muscle (data not shown). We conclude that the peptides are internalized in different cell types and that they are not degraded during incubation.

\subsubsection{YB-1 sequence appears to restrict to cell cytoplasm}

Having demonstrated internalization of all the proteins, we then investigated the localization of these proteins within cells by immunocytochemistry and indirect immunofluorescence. Cells grown on coverslips were incubated with $40 \mu \mathrm{g} / \mathrm{ml}$ of the fusion proteins for $3 \mathrm{hrs}$. and $18 \mathrm{hrs}$., for analysis by confocal microscopy. The cells were fixed and anti-GFP was used as primary antibody followed by FITC conjugated antirabbit IgG as the secondary antibody. By visual inspection of the confocal images we found that approximately $70 \%$ of the cells had taken up the protein by $18 \mathrm{hrs}$. The results indicate that the APGFP is internalized and distributed throughout the cell, localizing in both nucleus and cytoplasm (Fig. 2.7, panels A and E). In contrast, all the three YB-1 fusion proteins are seen localized only in the cytoplasm (Fig. 2.7, panels B to D and F to $\mathrm{H})$. We found that the levels of internalized fusion proteins seen in the cells at $18 \mathrm{hrs}$. are significantly higher than those seen at the $3 \mathrm{hr}$. time point. Similar results were obtained with primary cultures of rat aortic smooth muscle cells (RASM cells), indicating that it is not unique to rat hepatoma cells (data not shown). 


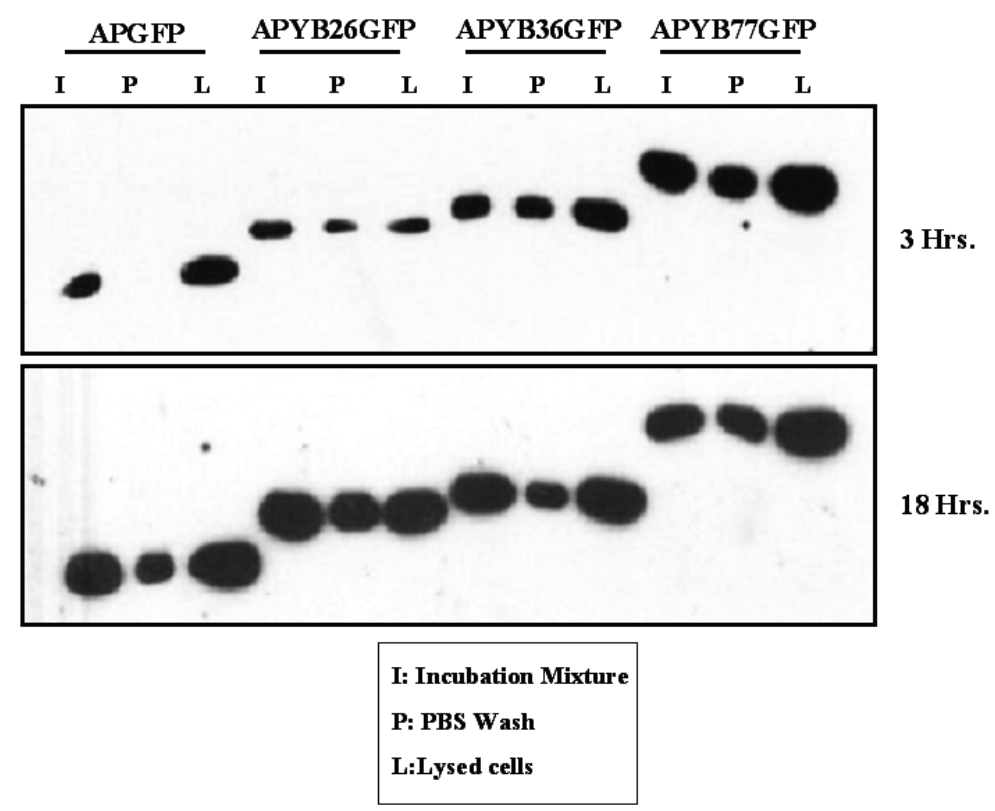

Fig. 2.6 APYBGFP and APGFP fusion proteins get internalized and are stable inside the cells

Rat hepatoma cells at $\sim 70 \%$ confluency were incubated with $40 \mu \mathrm{g} / \mathrm{ml}$ of each fusion protein for 3 and $18 \mathrm{hrs}$. Immunoblot analysis of whole cell extracts with polyclonal antibody against GFP shows the uninternalized peptides in the incubation mixture (I), the non-specifically bound peptides in the PBS wash $(\mathrm{P})$ and the internalized peptides in the lysed fractions (L). Note that all four fusion proteins show internalization and stability at the $3 \mathrm{hrs}$. and $18 \mathrm{hrs}$. time points. 

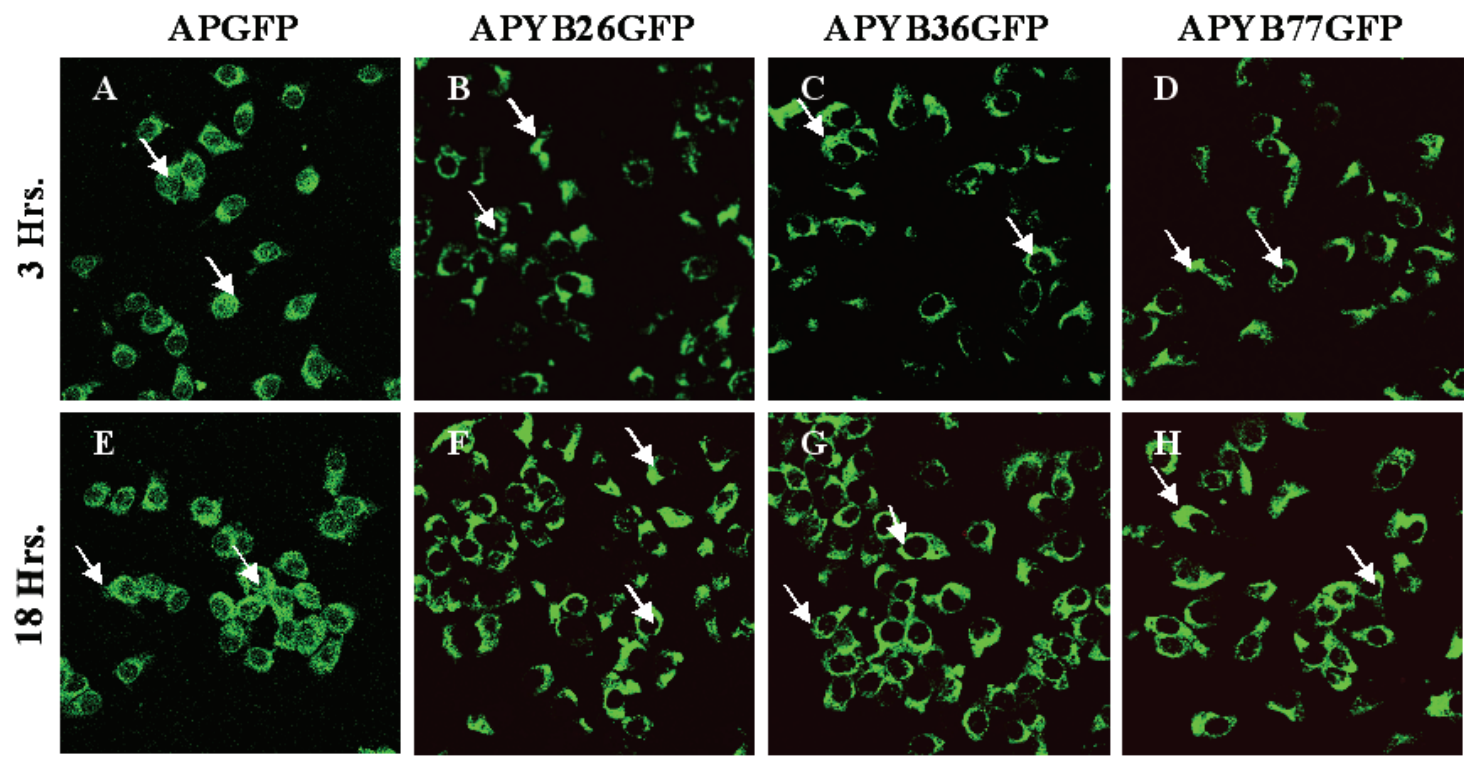

Fig. 2.7 APYBGFP fusion proteins are localized in cell cytoplasm

Rat hepatoma cells grown on cover slips to $\sim 70 \%$ confluency were incubated with 40 $\mu \mathrm{g} / \mathrm{ml}$ of each fusion protein for 3 and $18 \mathrm{hrs}$. for immunocytochemistry and indirect immunofluorescence analysis of sub-cellular distribution of the proteins. Cells were fixed with $4 \% p$-formaldehyde, blocked with 3\% goat serum and BSA and probed for the fusion proteins with antibody against GFP and FITC labeled secondary antibody. The sub-cellular distribution of the proteins analyzed by confocal microscopy is indicated by the arrows. Cells not incubated with the proteins acted as a negative control and these slides were used for optimization to minimize the non-specific binding and background fluorescence. The APYB26GFP (panels B and F), APYB36GFP (panels C and G), and APYB77GFP (panels D and H) proteins were seen exclusively in the cytoplasm, whereas APGFP was seen in both nucleus and cytoplasm (panels A and E). 
In order to confirm this result, we performed western blotting analysis on the nuclear and cytoplasm extracts of incubated rat hepatoma cells. We detected GFP signal only in the cytoplasmic extracts of cells incubated with the APYBGFP proteins even after $18 \mathrm{hrs}$. whereas the signals were seen in both nuclear and cytoplasmic extracts of APGFP incubated cells (Fig. 2.8). We conclude that the presence of YB-1 sequences in the fusion proteins is responsible for their restricted localization to cytoplasm of cells and that the initial proline rich 26 amino acids of YB-1 N-terminal sequence is sufficient for this differential localization.

\subsubsection{Effect of external stimuli on the localization of APYBGFP proteins}

Previous studies showed that cells exposed to extracellular stimuli like U.V. irradiation (Koike et al., 1997), anticancer drugs (Kohno et al., 2003), hyperthermia (Stein et al., 2001) or viral infection (Kohno et al., 2003) resulted in the translocation of YB-1 from the cell cytoplasm to nucleus. In order to investigate if the above-mentioned stimuli resulted in nuclear translocation of the YB-1 fusion proteins, rat hepatoma cells incubated with the fusion peptides were stimulated with either $20 \% \mathrm{FBS}$ or U.V. irradiation $\left(40 \mathrm{~J} / \mathrm{m}^{2}\right)$ in protein free medium for 3 and $18 \mathrm{hrs}$. respectively. For $20 \% \mathrm{FBS}$ stimulation, after fusion protein incubation in normal medium, the cells were incubated with protein free medium containing $20 \%$ FBS for different time periods. For UV stimulation, after the fusion protein incubations, the cells were treated with a total of $40 \mathrm{~J} / \mathrm{m}^{2}$ of UV irradiation in a CAMAG chamber and then placed in the incubator in normal protein free medium for the two time periods. Nuclear and cytoplasmic extracts of these cells were used for western blotting with GFP antibody. Serum stimulation resulted in about 25 to $28 \%$ of nuclear translocation of all three APYBGFP proteins after $3 \mathrm{hrs}$ and about 30 to $35 \%$ after $18 \mathrm{hrs}$. (Fig. 2.9, panels 1 and 2). U.V. irradiation stimuli resulted in nuclear translocation of about $35 \%$ APYB77GFP protein in $3 \mathrm{hrs}$ and about 38\% in 18 hrs. (Fig. 2.9, panels 3 and 4), whereas APYB26GFP and APYB36GFP were seen only in the cytoplasmic extracts at both time points (Fig. 2.9, panels 3 and 4). These results suggest that full length YB-1 N-terminus is required for the translocation of YB-1 from cytoplasm to nucleus under U.V. stimulation. APGFP was detected in both nuclear and cytoplasmic extracts under both stimuli.

In order to further confirm these results, we performed immunocytochemistry on cells grown on cover slips and incubated similarly. We detected the presence of about 32$37 \%$ nuclear translocation all three APYBGFP proteins in $3 \mathrm{hrs}$ and about $38-39 \%$ after $18 \mathrm{hrs}$. under serum stimuli (Fig. 2.10, panels B to D and F to H), thereby confirming our immunoblotting results. In case of U.V. stimulated cells, we detected about $32 \%$ nuclear APYB77GFP in $3 \mathrm{hrs}$ and about $39 \%$ in $18 \mathrm{hrs}$. (Fig. 2.11, panels D and H), whereas, APYB26GFP and APYB36GFP were seen only in nucleus (Fig. 2.11, panels B, C, F and $\mathrm{G})$. APGFP in both instances was seen both in nucleus and cytoplasm. 


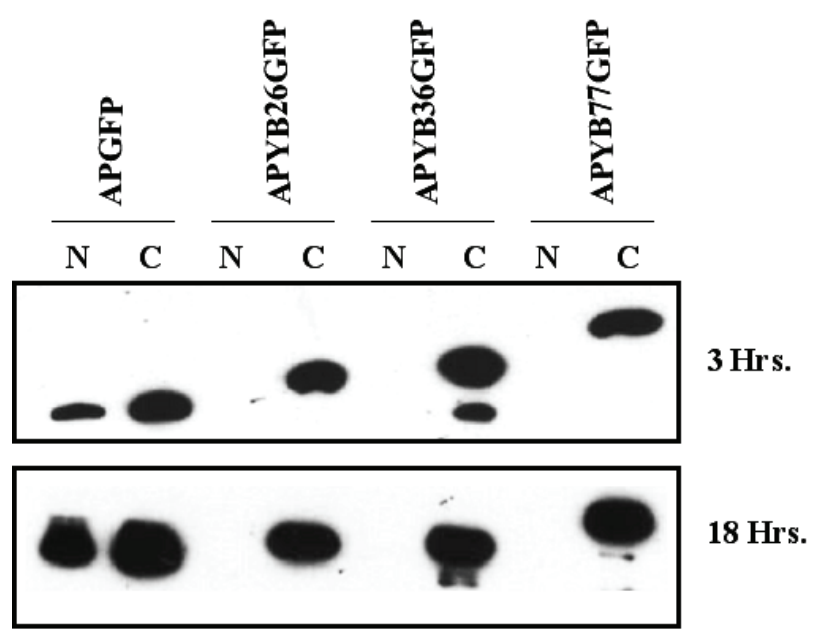

$\mathrm{N}$ : Nuclear extract

C: Cytoplasmic extract

\section{Fig. 2.8 Cytoplasmic localization of the APYBGFP fusion proteins confirmed by} western blotting

Rat hepatoma cells at $\sim 70 \%$ confluency were incubated with $40 \mu \mathrm{g} / \mathrm{ml}$ of each fusion protein for 3 and $18 \mathrm{hrs}$. Their nuclear (N) and cytoplasmic (C) extracts were analyzed for the fusion protein distribution by immunoblotting using polyclonal GFP antibody. Note that the three APYBGFP proteins were detected only in cytoplasmic extracts of cells at both time points. In contrast, APGFP was detected in both nuclear and cytoplasmic extracts. This confirms the results got from the immunocytochemistry experiment shown in Fig 2.7. 


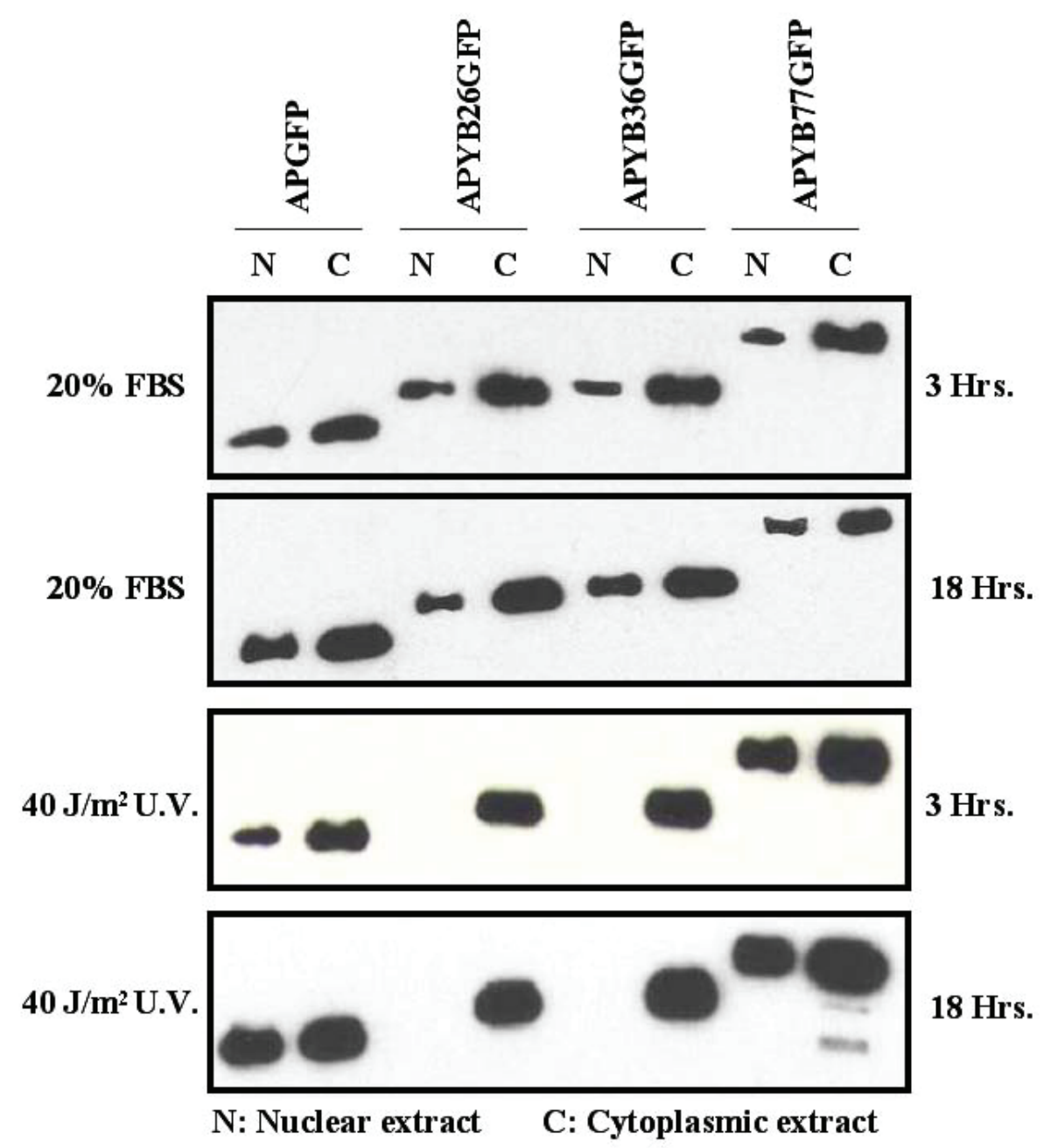

Fig. 2.9 Localization of the APYBGFP proteins in rat hepatoma cells changes in response to different stimuli

To investigate the sub-cellular localization of the fusion proteins after serum or UV stimulation, rat hepatoma cells at $\sim 70 \%$ confluency were incubated with $40 \mu \mathrm{g} / \mathrm{ml}$ of each fusion protein for $3 \mathrm{hrs}$. and then stimulated with $20 \% \mathrm{FBS}$ or $40 \mathrm{~J} / \mathrm{m}^{2} \mathrm{U}$.V. irradiation. Serum stimulation was provided by incubating cells in a protein free medium containing $20 \%$ FBS for 3 and $18 \mathrm{hrs}$. UV stimulation was provided by incubating the cells in protein free medium in a CAMAG UV chamber for total irradiation of $40 \mathrm{~J} / \mathrm{m}^{2}$ followed by incubation in the incubator for 3 and $18 \mathrm{hrs}$. Their nuclear $(\mathrm{N})$ and cytoplasmic (C) extracts were analyzed by immunoblotting with GFP antibody. Note that under serum stimuli (top two panels), about 25-28\% nuclear translocation of all APYBGFP fusion proteins was seen after $3 \mathrm{hrs}$ incubation and about $30-35 \%$ after $18 \mathrm{hrs}$. UV stimuli (botton two panels) resulted in about 35\% nuclear translocation of only APYB77GFP after $3 \mathrm{hrs}$. and about $38 \%$ after $18 \mathrm{hrs}$.. APGFP was detected in both nuclear (N) and cytoplasmic extracts (C) irrespective of the stimuli provided. 

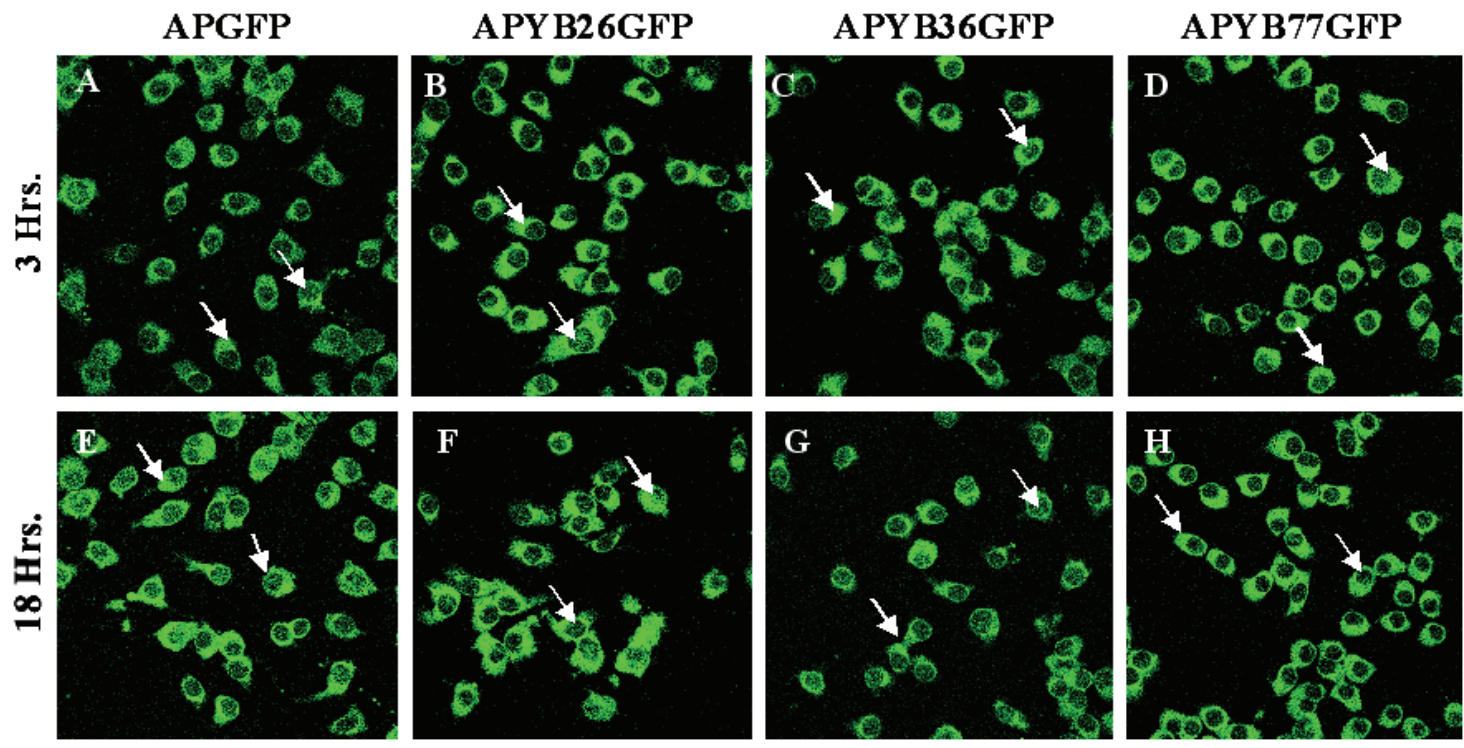

Fig. 2.10 APYBGFP fusion proteins partially translocated to the nucleus following $20 \%$ FBS treatment

Rat hepatoma cells grown on cover slips to $\sim 70 \%$ confluency were incubated with 40 $\mu \mathrm{g} / \mathrm{ml}$ of each fusion protein for $3 \mathrm{hrs}$. and then stimulate with serum by incubation in a protein free medium containing 20\% FBS for 3 and $18 \mathrm{hrs}$. for immunocytochemistry and indirect immunofluorescence analysis of sub-cellular distribution of the proteins under serum stimuli. Cells were fixed with $4 \% p$-formaldehyde, blocked with $3 \%$ goat serum and BSA and probed for the fusion proteins with antibody against GFP and FITC labeled secondary antibody. The sub-cellular distribution of the proteins analyzed by confocal microscopy is indicated by the arrows. Cells not incubated with the proteins acted as a negative control and these slides were used for optimization to minimize the non-specific binding and background fluorescence. About 32-37\% nuclear translocation of all three APYBGFP fusion proteins was seen after $3 \mathrm{hrs}$ (panels B, C and D) and about 38-39\% after 18 hrs. (panels F, G and H). The localization of APGFP did not change in comparison to the unstimulated cells (panels A and E). This confirms the western blotting result seen in Fig. 2.9. 

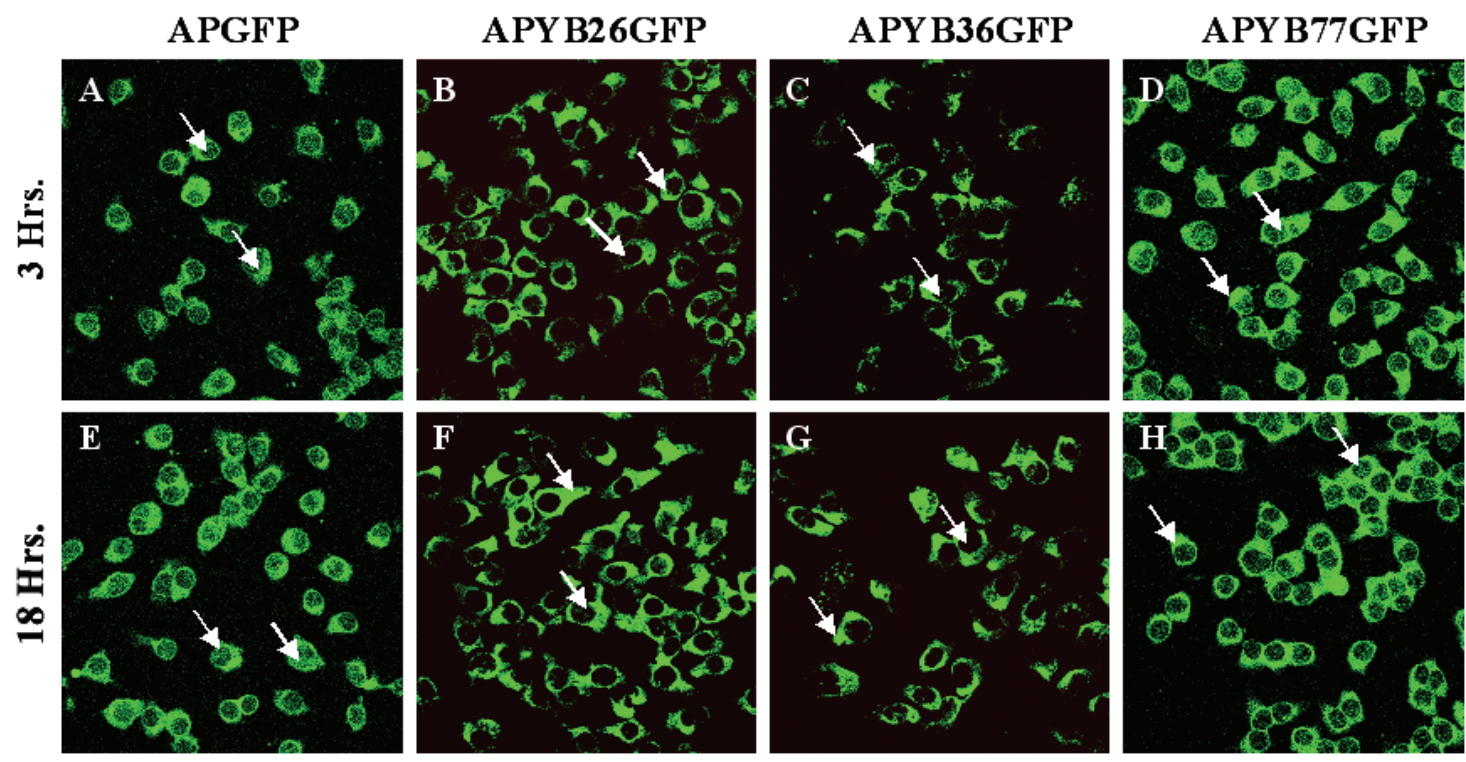

\section{Fig. 2.11 APYB77GFP gets partially translocated to the nucleus under UV treatment}

Rat hepatoma cells grown on cover slips to $\sim 70 \%$ confluency were incubated with 40 $\mu \mathrm{g} / \mathrm{ml}$ of each fusion protein for $3 \mathrm{hrs}$. and then stimulate with $40 \mathrm{~J} / \mathrm{m}^{2} \mathrm{U} . \mathrm{V}$. irradiation in a CAMAG UV chamber followed by incubation in a protein free medium containing for 3 and $18 \mathrm{hrs}$. for immunocytochemistry and indirect immunofluorescence analysis of subcellular distribution of the proteins under UV stimuli. Cells were fixed with $4 \% p$ formaldehyde, blocked with 3\% goat serum and BSA and probed for the fusion proteins with antibody against GFP and FITC labeled secondary antibody. The sub-cellular distribution of the proteins analyzed by confocal microscopy is indicated by the arrows. Cells not incubated with the proteins acted as a negative control and these slides were used for optimization to minimize the non-specific binding and background fluorescence. About $32 \%$ APYB77GFP was seen translocated to the nucleus after $3 \mathrm{hrs}$ (panel D) and about 39\% after $18 \mathrm{hrs}$ (panel H), whereas APYB26GFP and APYB36GFP were seen localized only in the cytoplasm (panels B and F and C and G). Localization of APGFP is comparable to that of the unstimulated cells (panels A and E). This confirms the western blotting result seen in Fig. 2.9. 


\subsubsection{Effect of APYBGFP proteins on cell cycle}

Cell cycle progression is mainly controlled by several key checkpoints, including G1/S checkpoint, S-phase DNA damage checkpoint and G2/M spindle integrity checkpoint. Previously reported defects in heterozygous mutant DT40 cells resembled the ones typical of cells with a defect at G2/M phase of cell cycle (Swamynathan et al., 2002). In order to study the effects of internalization of the proteins into rat hepatoma cells, we had to ensure that the fusion proteins are stable inside the cells for prolonged time periods. Therefore a preliminary experiment was performed, in which cells were incubated with the fusion proteins as described previously, for 24, 48 and $72 \mathrm{hrs}$. Whole cell lysates of the incubated cells were prepared and analyzed to investigate the stability of the internalized proteins. The principle here is that the stable proteins should produce a single band on the exposed film, whereas unstable proteins should show more than one bands, formed by degradation of the protein. We detected single bands, indicated by the GFP signals seen at full length sizes of all four proteins (Fig. 2.12). Among the three replicate experiments done to confirm the stability, only one showed a slight degradation of APYB77GFP. However the degraded product was quantitatively very low in comparison to the full length protein. The other two replicates did not show any degradation. Therefore we conclude that the proteins are stable atleast till the $72 \mathrm{hrs}$. time point of incubation.

In order to investigate if the cell cycle abnormalities are due to the $\mathrm{N}$-terminal YB-1 proteins, rat hepatoma cells were incubated with $40 \mu \mathrm{g} / \mathrm{ml}$ of each of the APYBGFP and APGFP proteins, respectively, for 24, 48 and $72 \mathrm{hrs}$. Half of each of the harvested samples was processed for propidium iodide staining and cell cycle analysis as described in methods. At any given time point in cell cycle in actively proliferating cells, a very high proportion of the cells are in the G1 phase and lesser than 10\% in the G2 phase. FACS analysis of the cells incubated with the APYB77GFP protein indicated that about $32 \%$ of cells were in G2/M phase of cell cycle by $72 \mathrm{hrs}$ (Fig. 2.13). More than $24 \%$ of cells were in G2/M phase when treated with APYB26GFP (Fig. 2.13). In contrast, the APYB36GFP incubated cells showed only $8 \%$ to $12 \%$ cells in the G2/M phase of cell cycle (Fig. 2.13). Control treatment with APGFP showed only $4-7 \%$ of cells in the $\mathrm{G} 2 / \mathrm{M}$ phase, which is comparable to the proportion of $\mathrm{G} 2 / \mathrm{M}$ phase cells in unincubated samples (Fig. 2.13). All reported differences are significant to $\mathrm{P}<0.0005$. We conclude that the APYBGFP incubation resulted in a significant cell cycle arrest at the $\mathrm{G} 2 / \mathrm{M}$ phase.

The remaining half of the samples were used to quantitate the GFP positive cells (Table 2.1) and the mean GFP fluorescence of each sample (Table 2.2). Results indicate that in all treatments comparable percentage of cells show fusion protein internalization. The mean GFP fluorescence seen in the four samples at each time point was also similar. This suggests that the differences in proportions of G2/M phase cells was not due to variation in the number of fusion protein-positive cells or amount of the internalized fusion proteins, but due to the presence of YB-1 N-terminal sequences in the three APYBGFP clones. These results render support for our hypothesis that the introduction of YB-1 N-terminal proteins into cells causes a defect in the G2/M phase of cell cycle. 


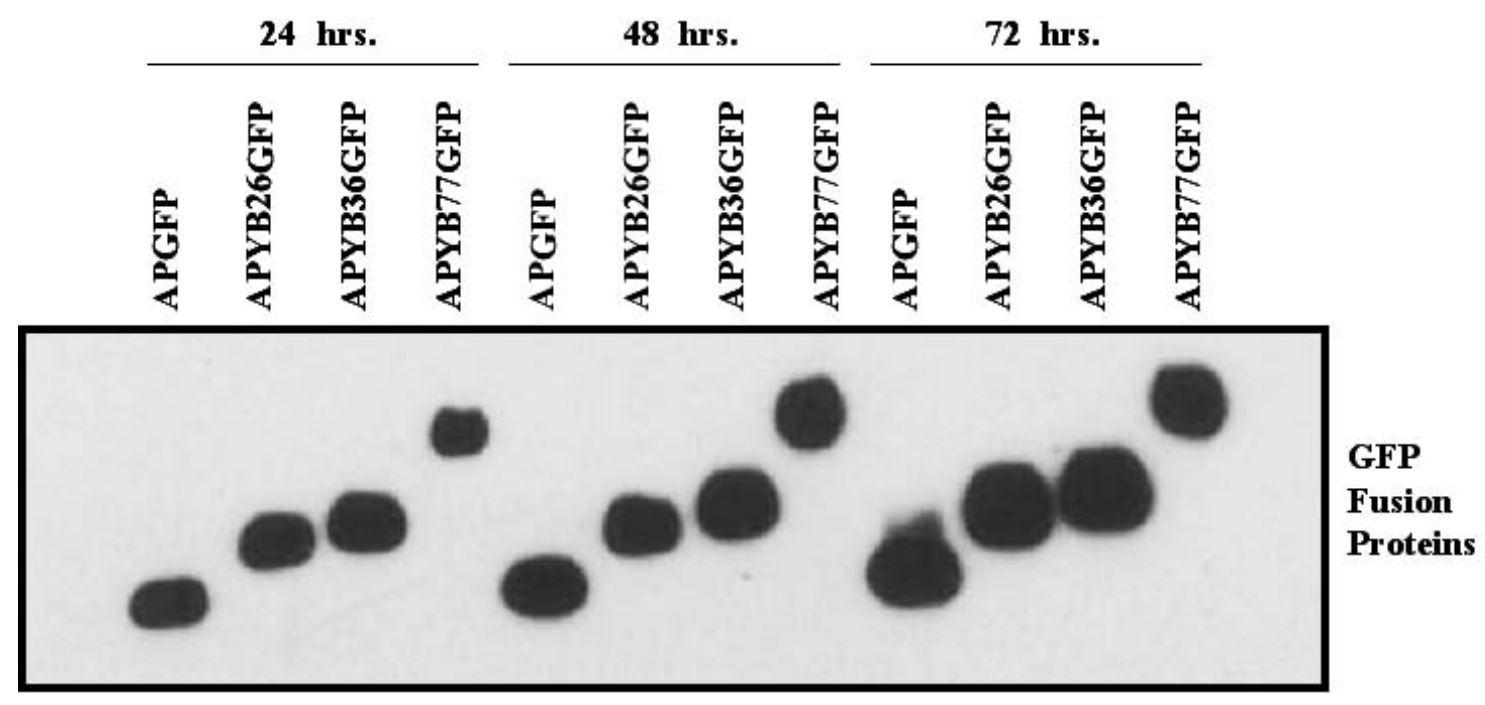

Fig. 2.12 APYBGFP fusion proteins are stable until the 72 hrs. time point of incubation

In order to investigate the stability of the proteins uopto $72 \mathrm{hrs}$ of incubation, rat hepatoma cells at $\sim 70 \%$ confluency were incubated with $40 \mu \mathrm{g} / \mathrm{ml}$ of each fusion protein for 24, 48 and $72 \mathrm{hrs}$. A representative blot from the immunoblotting analysis of whole cell extracts with polyclonal antibody against GFP shows that all proteins are intact even after $72 \mathrm{hrs}$. of incubation. 


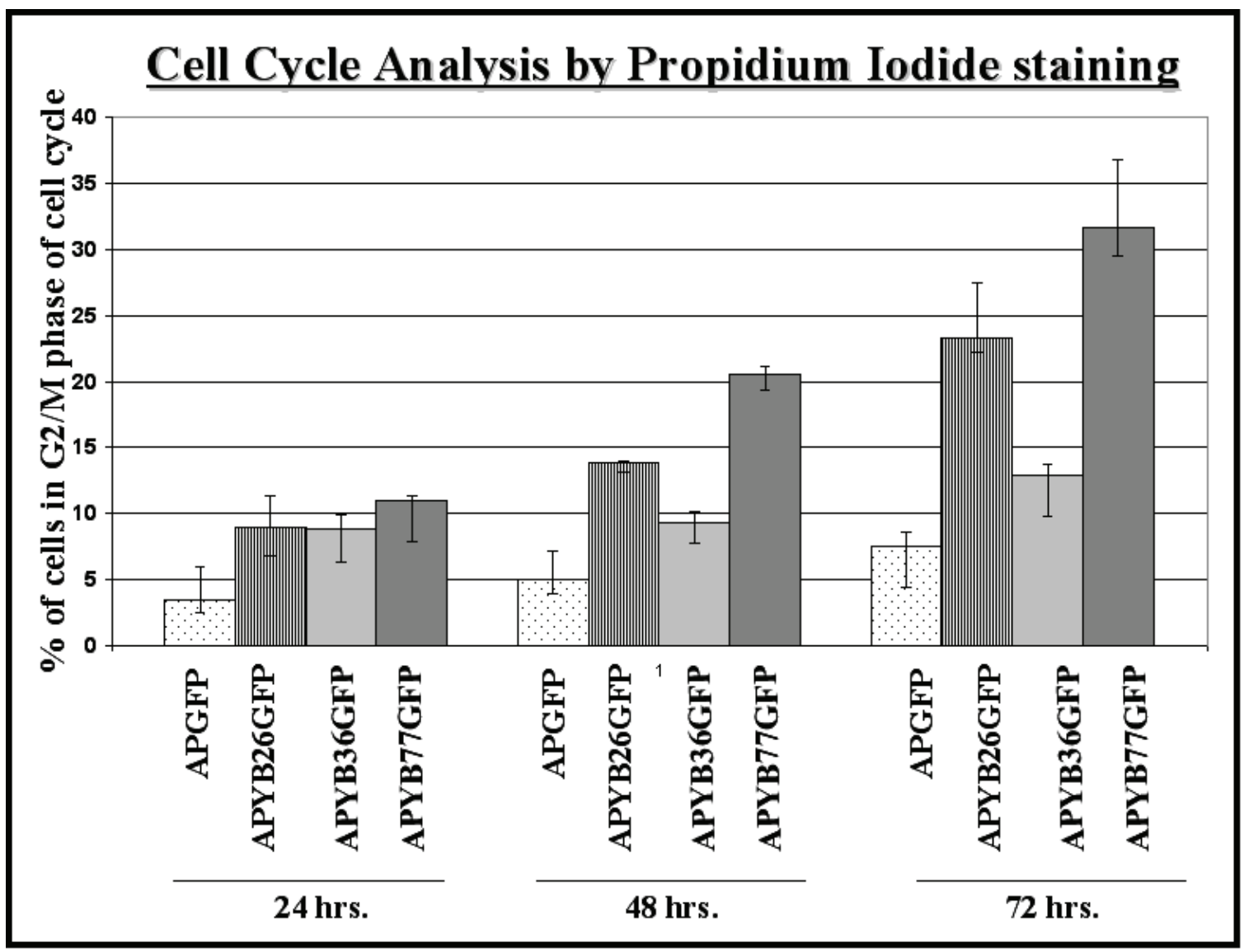

Fig. 2.13 Cells incubated with the APYBGFP proteins show cell cycle arrest at the G2/M phase of cell cycle

Exponentially growing rat hepatoma cells incubated with $40 \mu \mathrm{g} / \mathrm{ml}$ of the APYBGFP and APGFP proteins for 24, 48 and $72 \mathrm{hrs}$. were harvested and stained with propidium iodide. FACS analysis indicated that APYB77GFP incubation resulted in about $12-32 \%$ cells in the G2/M phase of cell cycle and APYB26GFP incubation led to $8-23 \% \mathrm{G} 2 / \mathrm{M}$ phase cells. This was significantly higher than unincubated cells (data not shown) or APGFP incubated cells at all three time points. Only $8-12 \% \mathrm{G} 2 / \mathrm{M}$ phase cells were detected in case of APYB36GFP incubation. All reported differences are significant to $\mathrm{P}<0.0005$. 
Table 2.1 GFP positive cell \% in cell cycle assay as determined by FACS analysis

\begin{tabular}{ccccc}
\hline Time & APGFP & APYB26GFP APYB36GFP APYB77GFP \\
\hline 24 hrs. & 67 & 69.6 & 69.9 & 70.9 \\
48 hrs. & 77.8 & 82.1 & 77.8 & 79.6 \\
72 hrs. & 85.8 & 86.4 & 84.5 & 86.5 \\
\hline
\end{tabular}

This table shows the \% of GFP positive cells from a representative experiment. We detected comparable $\%$ of GFP positive cells in all four types of fusion protein incubated samples at a given time point.

Table 2.2 Mean GFP fluorescence in cell cycle assay as determined by FACS analysis

\begin{tabular}{ccccc}
\hline Time & APGFP & \multicolumn{4}{c}{ APYB26GFP APYB36GFP APYB77GFP } \\
\hline 24 hrs. & 3753 & 3027 & 3417 & 3848 \\
48 hrs. & 4125 & 4347 & 4155 & 4242 \\
72 hrs. & 4952 & 6327 & 6018 & 4895 \\
\hline
\end{tabular}

This representative table shows that all the samples used in the experiment for cell cycle analysis have nearly the same amounts of protein uptake at any given time point, measured in terms of the mean fluorescence. 


\subsubsection{YB-1 amino terminal domain is involved in significant apoptosis}

Reduction in levels of YB-1 results in significant cell death. A recent study demonstrated that when YB-1 levels are downregulated, an increased rate of apoptotic cell death was observed (Shibahara et al., 2004), thereby showing a direct relation between YB-1 levels and apoptosis. Lately it was shown that siRNA mediated knockdown of YB-1, led to growth arrest and induction of apoptosis in multiple myeloma cells (Chatterjee et al., 2007). Previous studies from our laboratory (Swamynathan et al., 2002) also showed apoptosis in heterozygous mutant DT40 cells and that this might be due to a dominant negative effect of a potential truncated protein formed. Therefore we decided to study if apoptosis can be seen in cells with internalized APYBGFP proteins.

One of the hallmarks of apoptotic cells is the display of phosphotidylserine on their cell surfaces, which can be detected by using a fluorescent dye conjugated Annexin V. We incubated cells with $40 \mu \mathrm{g} / \mathrm{ml}$ of each the four fusion proteins respectively for 24,48 and $72 \mathrm{hrs}$. and harvested the cells. Half of each of these samples were processed for Annexin V staining as described previously in methods and analyzed by FACS analysis. Three independent experiments were performed for this analysis. As seen from Fig. 2.14, APGFP incubated cells show negligible apoptosis at all three time points. In contrast, a high proportion of apoptotic cells were detected in APYB77GFP incubated cells. As early as $24 \mathrm{hrs}$ of incubation, about $8-9 \%$ of the cells were apoptotic, increasing to 17 $18 \%$ at the $48 \mathrm{hrs}$. time points and an average of about $22-23 \%$ by the $72 \mathrm{hrs}$. time points. APYB36GFP incubated cells also show apoptosis reaching to about $11-12 \%$ by the $72 \mathrm{hrs}$. time point, which though was higher than the control, was still statistically very low as compared to the APYB77GFP incubation. Most interestingly, APYB26GFP incubated cells show approximately $6-7 \%$ of apoptosis at $24 \mathrm{hrs}$., about $9-10 \%$ by the 48 hrs. and the proportion increased to about $18-19 \%$ by 72 hrs (Fig. 2.14). All differences reported are significant to $\mathrm{P}<0.0007$.

In order to confirm that the apoptosis seen in the above experiment was not due to differences in the number of GFP positive cells or the quantitative uptake of the proteins, we quantitated the proportion of GFP positive cells and the mean GFP fluorescence. Results indicate that the \% of GFP positive cells and the mean GFP fluorescence is approximately the same in all four proteins' incubation over the three time points (Table 2.3 and 2.4).

Another hallmark of apoptosis is karyorrhexis, during which the nuclear envelope becomes discontinuous and the DNA gets fragmented giving a characteristic ladder like appearance. Cells were incubated with proteins for the three time periods, DNA was isolated and analyzed on a $1.2 \%$ agarose gel. DNA fragmentation pattern in the form of a 180-200 bps ladder was clearly observed in the lanes with DNA from the APYBGFP proteins incubated cells, whereas cells incubated with the control protein, APGFP, showed intact full length DNA (Fig. 2.15). We observed that there is an increasing level of the laddered pattern seen in all the 3 protein incubated cells for time points 24 to 72 hrs., signifying an increase in the proportion of apoptotic cells (Fig. 2.15). However DNA laddering in the APYB36GFP incubated cells was less as compared to the APYB26GFP 


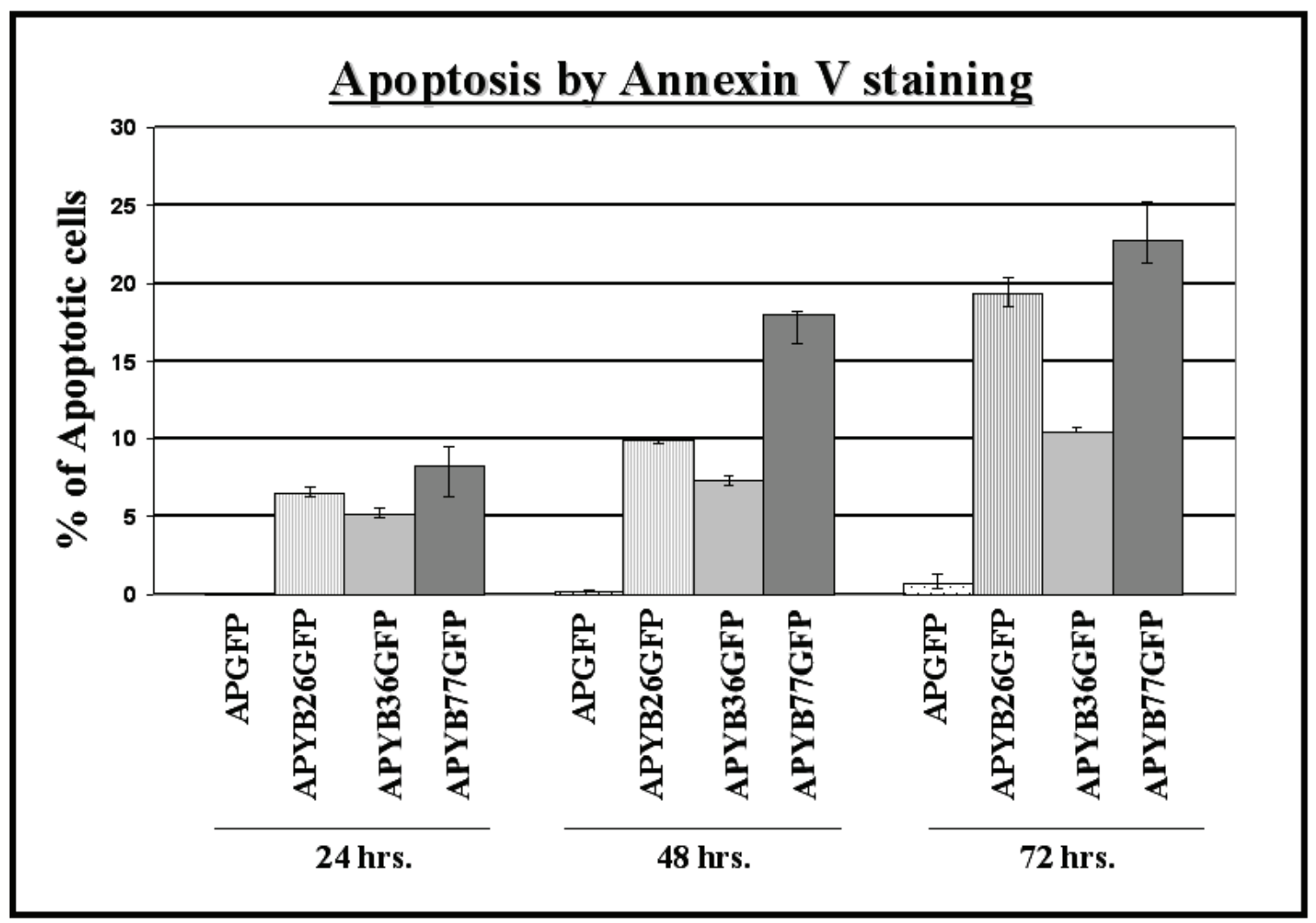

Fig. 2.14 APYBGFP protein incubation results in significant apoptosis by Annexin $\mathrm{V}$ staining and FACS analysis

Exponentially growing rat hepatoma cells incubated with $40 \mu \mathrm{g} / \mathrm{ml}$ of the APYBGFP and APGFP proteins for 24, 48 and $72 \mathrm{hrs}$. were harvested and stained with FITC conjugated Annexin V. FACS analysis indicated that APYB77GFP incubation resulted in about 8 to $23 \%$ apoptotic cells and APYB26GFP incubation led to 7 to $19 \%$ apoptotic cells. Only 5 to $10 \%$ apoptotic cells were detected in case of APYB36GFP incubation. The apoptosis in APGFP incubated cells was statistically insignificant. All reported differences are significant to $\mathrm{P}<0.0007$. 
Table 2.3 GFP positive cell \% in apoptotic assay as determined by FACS analysis

\begin{tabular}{ccccc}
\hline Time & APGFP & APYB26GFP & APYB36GFP & APYB77GFP \\
\hline 24 hrs. & 77.3 & 77.1 & 77.0 & 77.7 \\
48 hrs. & 79.8 & 77.8 & 79.7 & 79.8 \\
72 hrs. & 81.3 & 82.9 & 83.6 & 84.0 \\
\hline
\end{tabular}

The $\%$ of GFP positive cells is listed out from one representative experiment. We detected comparable \% of GFP positive cells in all four types of fusion protein incubated samples at a given time point.

Table 2.4 Mean GFP fluorescence in apoptotic assay as determined by FACS analysis

\begin{tabular}{ccccc}
\hline Time & APGFP & APYB26GFP & APYB36GFP & APYB77GFP \\
\hline 24 hrs. & 4346 & 5376 & 4923 & 4856 \\
48 hrs. & 5644 & 5554 & 5430 & 5546 \\
72 hrs. & 5962 & 6349 & 6556 & 5675 \\
\hline
\end{tabular}

A representative table of the mean fluorescence of all the samples analyzed for apoptosis, shows equivalent mean fluorescence among all samples at a given time point. 


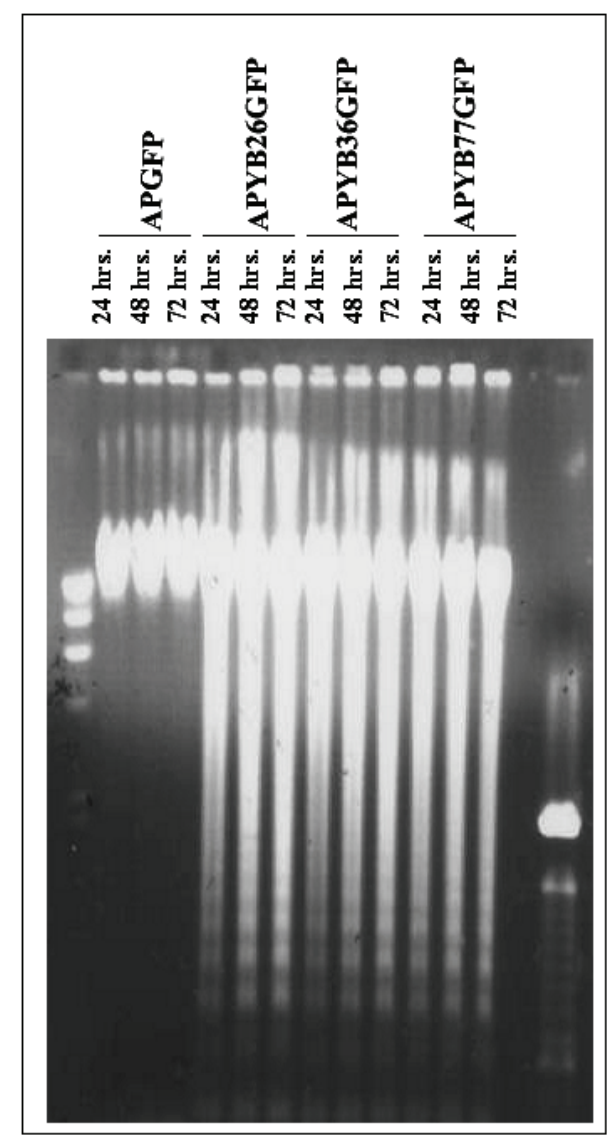

Fig. 2.15 APYBGFP protein incubation results in significant DNA fragmentation (Karyorrhexis)

Cellular DNA was isolated from cells incubated with the three APYBGFP or APGFP proteins respectively for 24, 48 and $72 \mathrm{hrs}$. time points. Equivalent amounts of DNA from these samples were loaded onto a $1.2 \%$ agarose gel. DNA ladder as expected of apoptotic cells was detected in DNA from cells incubated with the three APYBGFP proteins but not from the APGFP incubated cells. 
and APYB77GFP incubated cells, confirming our observations in the previous experiment by Annexin V staining.

A third hallmark of apoptotic cells is pyknosis, during which the chromatin undergoes condensation into compact patches against the nuclear envelope and the nucleus breaks into several discrete chromatin bodies or nucleosomal units due to the degradation of DNA. We analyzed the nuclei of APYBGFP incubated cells by DAPI staining to investigate if they showed any pyknosis. We detected chromatin condensation and the broken down nucleus in cells treated with all the 3 APYBGFP proteins for 24 to $72 \mathrm{hrs}$. (Fig. 2.16, panels B to D, F to $\mathrm{H}$ and $\mathrm{J}$ to $\mathrm{L}$ ) whereas the nuclei of cells incubated with the control protein APGFP showed normal appearance (Fig. 2.16, panel A, E and I). Based on these observations we conclude that the YB-1 sequence in the three APYBGFP clones is responsible for the cells to undergo apoptosis.

\subsection{Discussion}

YB-1 expression is closely associated with cell proliferation. Previous studies from our laboratory showed that disruption of one allele of Chk-YB-1b gene in DT-40 cells resulted in abnormal phenotypes, including slower growth rate of cells, increased genomic DNA content that is associated with apoptosis and some morphological changes (Swamynathan et al., 2002). We hypothesized that the abnormalities seen may be due to the dominant negative effect exerted by a putative truncated protein product of the disrupted allele, which spans the YB-1 N-terminus. In this study we sought to test this hypothesis by investigating the effects of introduction of the N-terminal YB-1 sequences as exogenous proteins into rat hepatoma cells. The fusion proteins constructed to test our hypothesis were i) APYB77GFP, with the 77 amino acid N-terminal YB-1 sequence, till the Sty I site, ii) APYB36GFP with the internal deletion from 12-52, iii) APYB26GFP, with the proline rich 26 amino acid stretch and iv) APGFP, which was our control fusion protein.

We demonstrated that internalization of the four APGFP fusion proteins occurs at high efficiency in exponentially growing rat hepatoma cells with more than $70 \%$ cells showing the protein within $18 \mathrm{hrs}$. of incubation. Moreover as seen from the data, the proteins retained their stability even up to $72 \mathrm{hrs}$. of internalization. In contrast to the control fusion protein APGFP, which is seen in both cytoplasm and nucleus, the three APYBGFP fusion proteins showed localization restricted to the cytoplasm of cells. This result was in agreement with previous studies showing that the YB-1 carboxy-terminal domain harbors its nuclear localization signal and the cold shock domain contributes to its nuclear retention (Bader et al., 2005). Yet another study showed that the YB-1

carboxy-terminal domain binds to a yet unknown anchoring protein in the cytoplasm and thus retains the YB-1 there (Koike et al., 1997). However, the nucleocytoplasmic shuttling of YB-1 has not been clearly understood and our results add to the current knowledge in this field, by demonstrating that the YB-1 carboxy-terminus is not essential for its cytoplasmic retention. 


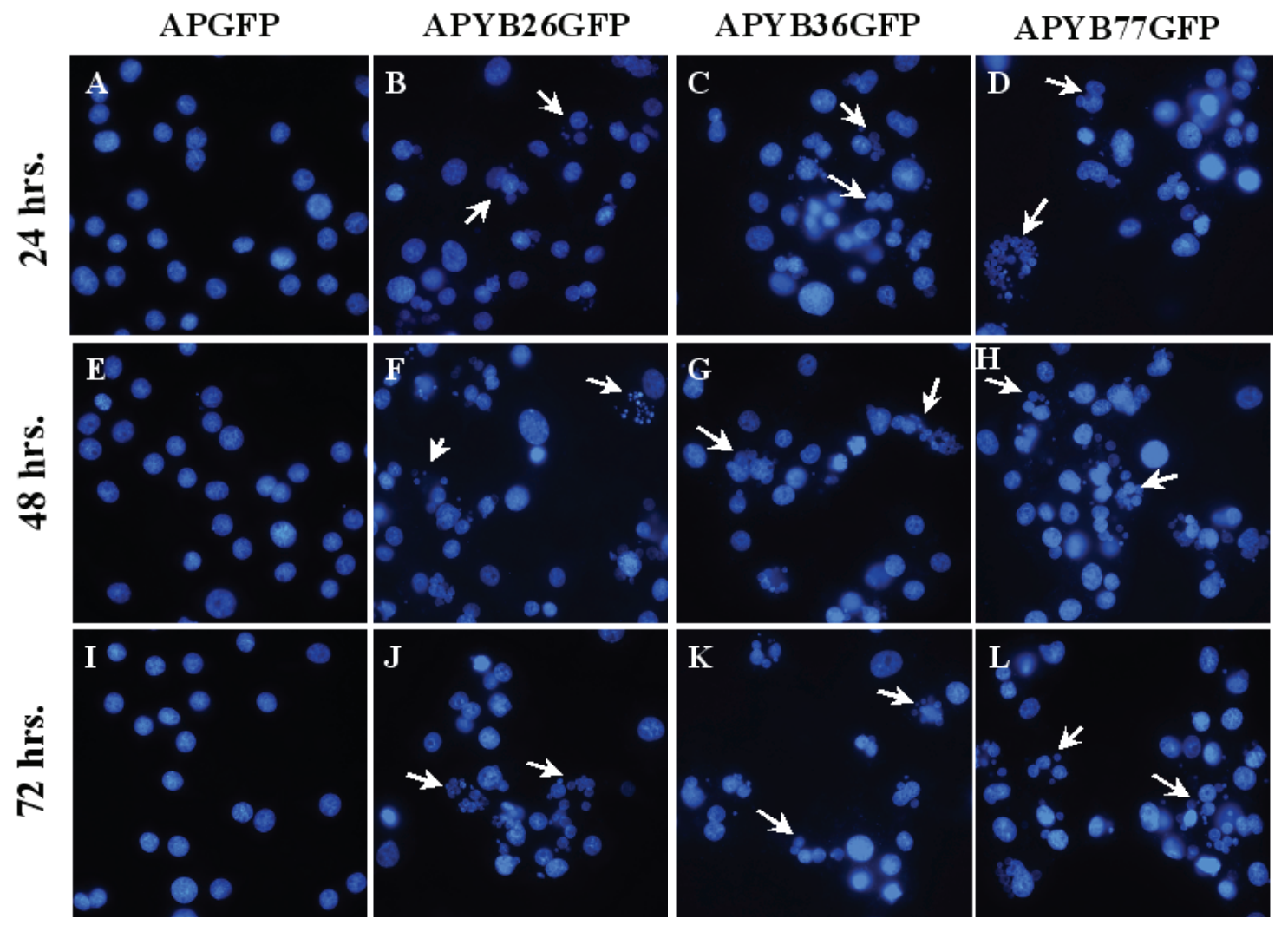

Fig. 2.16 APYBGFP protein incubation results in significant pyknosis

Exponentially growing rat hepatoma cells incubated with $40 \mu \mathrm{g} / \mathrm{ml}$ of the APYBGFP and APGFP proteins for 24, 48 and 72 hrs. were stained with DAPI. Fluorescence microscopy was used to analyze the stained nuclei of cells The arrows in panels B to D, F to $\mathrm{H}$ and $\mathrm{J}$ to $\mathrm{L}$ indicate the chromatin condensation and apoptotic nuclear bleb like formations in the nuclei of cells incubated with APYB26GFP, APYB36GFP and APYB77GFP proteins, whereas APGFP incubated cell nuclei (panels A, E and I) show normal appearance. 
Though YB-1 is predominantly a cytoplasmic protein, it has been reported that various genotoxic stresses like U.V. irradiation, hyperthermia, anticancer drugs, IF- $\gamma$ treatment and adenoviral infection result in its partial translocation to the nucleus (Higashi et al., 2003; Kohno et al., 2003; Raffetseder et al., 2003; Zhang et al., 2003). Here we showed that rat hepatoma cells stimulated with $20 \%$ FBS show partial nuclear translocation of the three APYBGFP proteins from cytoplasm. 20\% FBS was shown to stimulate YB-1 phosphorylation (Evdokimova et al., 2006), which reversed mRNA silencing by YB-1. It is likely that the mRNA free YB-1 gets translocated to the nucleus and therefore we detected the nuclear translocation of fusion proteins.

In contrast to the serum treatment, U.V. irradiation did not stimulate nuclear translocation of APYB26GFP and APYB36GFP. Only the APYB77GFP showed partial translocation from the cytoplasm to nucleus. Different genotoxic stresses activate different cellular responses to overcome stress. One previous study showed that nuclear translocation of YB-1 under UV stimuli is mediated by protein kinase C modulated mechanism (Koike et al., 1997). However the protein kinase C phosphorylation site on YB-1 is in its cold shock domain at amino acid position196, which is not present in the APYB77GFP protein. It has only one phosphorylation site at position 74 that is phosphorylated by a casein kinase II. So if this nuclear translocation is modulated by phosphorylation, it has to be at the position 74 and not 196. But it does not seem likely that this might be the mechanism of nuclear translocation in this instance, since the APYB36GFP has the amino acid 74 and yet it does not get translocated to the nucleus under UV stimuli. But the internally deleted amino acids from the APYB36GFP protein might have some effect on this process. The same UV stimulation experiment performed with the APYB77GFP protein with an mutation at the 74 amino acid can give a better idea is this nuclear translocation is mediated by phosphorylation at the position 74 amino acid by casein kinase II.

It has also been shown that only under UV irradiation, YB-1 forms a complex with p53/WRN, during the process of which it gets translocated into the nucleus (Guay et al., 2006). YB-1 N-terminus is implicated in its interaction with p53 (Okamoto et al., 2000). This is another likely mechanism, which might explain the nuclear translocation of only APYB77GFP under UV stimuli, but not APYB26GFP and APYB36GFP, which lack the full length N-terminus and therefore might not be able to form a complex with p53/WRN. UV irradiation also leads to YB-1 binding to its Y-box (Uchiumi et al., 1993; Asakuno et al., 1994). It is likely that this interaction is mediated only by the full length YB-1 $\mathrm{N}$-terminus. However YB-1 cold shock domain and C-terminal domains are also shown to be involved in binding with p53. So the mechanism of nuclear translocation of only APYB77GFP cannot be totally explained by its ability to bind to p53 and the inability of APYB26GFP and APYB36GFP for the same due to the lack of some residues. Analysis of nuclear translocation of YB-1 with truncated N-terminal proteins but possessing the CSD and CT domains can provide a better insight about the importance of the amino terminal residues for nuclear translocation under UV stimuli.

Incubation of cells with the APYB77GFP and APYB26GFP fusion proteins also resulted in significant cell cycle arrest at the G2/M phase. YB-1 has already been 
implicated in the prevention of premature cellular senescence (Lu et al., 2005). It is possible that due to the dominant negative effect exerted by the $\mathrm{N}$-terminal proteins, the cells lose their ability to divide. Our results and interpretations are concurrent with the previous knowledge available about YB-1. It is very likely that YB-1 can provoke cell division failure by its specific interaction with actin (Ruzanov et al., 1999), because actin filaments form contractile rings, which help cleave cells during cytokinesis (Robinson et al., 2004). YB-1 might also directly affect centrosome functions as it binds to the centrosome during mitosis (Janz et al., 2000). Centrosome controls cell exit from cytokinesis (Piel et al., 2001) and cytokinesis failure leads to chromosomal instability and aneuploidy (Lingle et al., 2002; Nigg, 2002), which might lead to a block in cell cycle and apoptosis.

Several reports have shown the relationship between the levels of YB-1 and induction of apoptosis (Schittek et al., 2007; Chatterjee et al., 2007). Our previous study showed that targeted disruption of one allele of YB-1 resulted in a small proportion of cells exhibiting apoptosis. In this study we show that the APYB77GFP and APYB26GFP incubated cells show significant apoptosis. This result supports our hypothesis that the defects seen in the heterozygous mutants in our previous study were the result of a dominant negative effect of the potential truncated protein.

We performed some preliminary experiments to investigate if the $\mathrm{G} 2 / \mathrm{M}$ phase arrested cells from the APYB77GFP or APYB26GFP incubations are the very population of cells that underwent apoptosis. For this purpose, we sorted out G2/M phase cells and then analyzed the proportion of Annexin V stained cells in this population. But due to the technical limitations of using propidium iodide and Annexin V in the same samples, the data obtained was not very conclusive (data not shown).

In contrast to the APYB77GFP and APYB26GFP incubated cells, the APYB36GFP incubated cells showed a lower percentage of cells blocked in the G2/M phase of cell cycle. Incubation with APYB36GFP also resulted in apoptotic cells, albeit much lesser in proportion to the percentage seen in case of the other two APYBGFP fusion proteins. APYB77GFP contains all the 77 amino acids of from the YB-1 $\mathrm{N}$-terminus. This includes the alanine - proline rich sequence SEAETQQPPAAPPAAPALSAADTKP, which is also present in the APYB26GFP. But only a part of it (SSEAEQQPP) is present in APYB36GFP. Since both APYB77GFP and APYB26GFP showed maximum effect on the cell cycle and apoptotic ability as compared to APYB36GFP, it is very likely that this defect in cell cycle and apoptotic ability of cells is directly or indirectly mediated by the presence of the proline rich PPAAPPAAPALSAADTK sequence.

There is a possibility that the YB-1 proline-alanine rich residues play a role in the fusion protein folding and due to their absence in the APYB36GFP, proper folding is either hindered or enhanced thereby leading to a difference in proportion of apoptotic cells. Proline residues are widely recognized as playing a special role in folding and unfolding transitions of globular protein molecules. But whether prolines help in stabilizing protein folding or results in its destabilization, is still not clear. Prolines were 
initially shown to destabilize folded conformations (Levitt, 1981). Later studies have shown that proline residues are important for proper protein folding (Kusano et al., 2001). Proline residues are shown to have opposite effects on fast and slow protein folding phases (Osvath et al., 2003). Therefore lack of many proline residues in the APYB36GFP might be the likely explaination behind the cell cycle arrest and apoptosis differences. $\mathrm{X}$-ray crystallography of the purified fusion proteins would provide us with more insight into the three dimensional structure of these proteins and the possible role of the proline alanine rich sequence in the protein conformation.

It is also likely that these proline residues are important for interaction of YB-1 with some other cell cycle proteins, which eventually help in mediating the apoptosis. Further on, in the next set of experiments, we show an interaction between the APYB77GFP / APYB26GFP proteins and Cyclin D1 and propose that the cells get blocked at the G2/M phase of cell cycle due to this interaction. Construction of a fusion protein with only the proline alanine rich sequence missing from APYB36GFP can throw light on the hypothesized importance of this sequence. Further experiments with serial deletion mutants of the YB-1 N-terminal sequence can provide further knowledge about the minimal YB-1 sequence/residues required for cell cycle arrest and apoptosis. Our final conclusion from this part of the project is that the amino-end domain of YB-1 is involved in regulating cell proliferation. 


\section{Chapter 3. YB-1 Interacts with Cyclin D1 in the Cytoplasm of Cells in G2/M Phase of Cell Cycle}

\subsection{Introduction}

Protein-protein interactions are intrinsic to virtually all cellular processes. Proteins rarely function individually and it has been proposed that all proteins in a given cell are connected with other proteins through an extensive network, where non-covalent interactions continuously associate and dissociate. Finding interactions between proteins involved in common cellular functions is a way to get a broader view of how they work cooperatively in a cell. YB-1 protein during its life span associates with many proteins, like transcription factors, RNA binding proteins, viral proteins and DNA repair proteins to yield functionally positive or negative influences.

It is likely that one of the mechanisms by which YB-1 is involved in cell proliferation is by its binding with one or more cell cycle proteins. Several cell cycle regulated genes contain Y-box binding sequence in their promoter or enhancer regions (Ladomery et al., 1995). YB-1 protein has already been shown to interact with some cell cycle proteins. For example, YB-1 interacts with p53 and this interaction is responsible for regulating the p53 gene expression (Okamoto et al., 2000). p53 has been shown to be involved in regulating centrosome duplication (Deng et al., 2000) and it is interesting to note that YB-1 also associates with the centrosome in mitotic cells (Janz et al., 2002; Bergmann et al., 2005).

By disrupting one allele of chicken YB-1 gene in DT-40 cells we reported many phenotypic and morphological abnormalities in the heterozygous mutants (Swamynathan et al., 2002). One striking difference between the wild type and mutant cells was downregulation of cyclin dependent kinase inhibitor, p21, in the mutant cells. p21 was shown to bind to and inhibit the activity of cyclin D1/CDK4 complex thus regulating cell cycle progression (Cheng et al., 1999; Gladden et al., 2005). Many groups have elucidated the involvement of YB-1 with cyclins at different levels of regulation including transcriptional and translational regulations. YB-1 contributes to transcriptional regulation of cyclin A and cyclin B1 genes (Jurchott et al., 2003). YB-1 knockout by siRNA in ovarian cancer cells upregulates cyclin B gene expression (Basaki et al., 2007). YB-1 binds to cyclin D1 mRNA resulting in its translational regulation (Evdokimova et al., 2006). Decreasing the levels of YB-1 protein by shRNA results in a decrease of cyclin D1 protein levels (Schittek et al., 2007). However, no study has shown a direct interaction of YB-1 with the cyclins at the protein level.

Cyclins are a family of proteins, involved in the progression of the cells through cell cycle. They are key regulators of cell cycle transition, whose abundance varies through cell cycle (Deanna et al., 1999). They were first identified in marine invertebrates as proteins whose accumulation and degradation oscillated during the cell cycle (Rosenthal et al., 1980). There are multiple cyclins like cyclin A, B1, C, D1, D2, D3, E, F, G, H, K and T, each with a specific function. 
D type cyclins (cyclins D1, D2 and D3) are regarded as essential links between cell environment and the core cell cycle machinery. Among all cyclin classes, these are the only ones that stand out as a very unique component of the cell cycle apparatus. Unlike other cyclins that fluctuate periodically during cell cycle progression, the extracellular environment controls D type cyclin levels. Thus, D type cyclins are induced by the mitogenic stimulation and their levels decline when the mitogens are withdrawn (Sherr et al., 1999). Their levels are also likely to be regulated by cell cycle dependent events (Stacey 2003).

The manner in which mammalian D type cyclins promote progression of cell cycle has lately been a topic of intense investigation. The $\mathrm{D}$ type cyclins form the regulatory subunit of the CDK4/6 kinase and function as critical mitogenic sensors that integrate the growth factor-initiated signals with cell cycle progression (Marshall, C., 1999). Most cells synthesize more than one D-type cyclin and certain cell types like epithelial cells in the mammary glands of pregnant mice depend almost exclusively on Cyclin D1 for their proliferation (Sicinski et al., 1995; Fantl et al., 1995). At the time of their discovery the D-type cyclins were immediately recognized to be growth factor responsive genes (Matsushime et al., 1991).

Cyclin D1, which is a prototype of the cyclin gene family, was independently identified through its ability to rescue G1 cyclin-deficient yeast strains (Xiong et al., 1991) and it was targeted at the breakpoints of chromosomal translocations in cancer cells, implicating it as a proto-oncogene (Motokura et al., 1991). Of the three D-type cyclins, cyclin D1 remains the most extensively studied, largely because of its frequent overexpression in human malignancy. It is encoded by the Bcl-1 gene and is an essential regulator of cell cycle progression. Aberrant induction of cyclin D1 activity is reported and well established in many human tumors (Peters, G., 1994; Gillett et al., 1994; Hall et al. 1996; Palmero et al., 1996; Arnold, A., 1995; Sherr, C.J., 1996 and 2000; Sherr et al., 2002; Diehl et al., 2002). It is shown to exert its effects on cell proliferation by integrating external signals (e.g., mitogens), with the cell cycle machinery (Peters, G., 1994; Palmero et al., 1996; Sherr, C.J., 1996 and 2000; Diehl et al., 2002; Lukas et al., 1996; Brown et al., 1998; Roussel et al., 1995).

Cyclin D1 expression is regulated by multiple mechanisms, including, transcription (Albanese et al., 1995; Winston et al., 1996), its own protein stability (Matsushime et al., 1991; Hashemolhosseini et al., 1998; Agami et al., 2000; Casanovas et al., 2000; Miyakawa et al., 2001; Guo et al., 2002), message turnover (Hashemolhosseini et al., 1998; Dufourny et al., 2000; Lin et al., 2000) and nucleocytoplasmic transport of the transcript and the protein (Rosenwald et al., 1995; Topisirovic et al., 2003). Cyclin D1 transcription is generally stimulated as a delayed early response to mitogenic signaling cascades (Sherr, C.J., 1995 and 1996). Its protein stability is regulated through the glycogen synthase kinase $3 \beta$-signal transduction pathways to coordinately enhance accumulation of cyclin D1 protein (Diehl et al., 1997 and 1998). Once synthesized cyclin D1 interacts with and activates CDK4 and CDK6 (Meyerson et al., 1994; Matsushime et al., 1992). CDK4/6-cyclin D1 complexes are both rate limiting and required for cell cycle 
progression. Thus, the intricate regulation of cyclin D1 expression and activity plays a critical role in ordered progression of cells through the cell cycle.

Cyclin D1 was shown to be one of the key regulators of G1/S transition (Johnson et al., 1999; Massague et al., 2004; Kastan et al., 2004). However, the expression levels of cyclin D1 in the G2 phase determine the fate of the next cell cycle (Guo et al., 2005). If Ras activity is abrogated for any reason during the S/G2 phase, Cyclin D1 induction during the G2 phase is suppressed and the cells get arrested immediately after mitosis, due to insufficient levels of cyclin D1 for entry into the subsequent S phase (Hitomi et al., 1999). Thus, even though cyclin D1 is important for the G1/S transition of cells (Baldin et al., 1993; Lukas et al., 1995; Ohtsubo et al., 1995), it is absolutely essential at the G2 phase for the cells to decide to complete the next division cycle (Hitomi et al., 2001; Stacey, 2003).

Striking similarities exist between YB-1 and cyclin D1. The levels of both proteins are upregulated in a variety of neoplasms (Kohno et al. 2003; Fu et al. 2004) including primary breast cancers, osteosarcomas, ovarian cancers, lung cancers, colon cancers, synovial sarcomas and parathyroid adenomas. They show similar cellular localization at the G2 phase of cell cycle. Although YB-1 is classified as a cytoplasmic protein and cyclin D1 a nuclear protein (Baldin et al., 1993), YB-1 is found in the nucleus in mid G1 phase to S phase (Jurchott et al 2003) at the same time with cyclin D1. Cyclin D1 relocates to the cytoplasm after the end of S phase progressing to the G2 phase (Stacey 2003; Gou et al., 2005), when YB-1 is also mostly concentrated in the cytoplasm. Cyclin D1 protein levels increase in G2 phase and are maintained through mitotic and G1 phases before its level goes down in the S phase. In fact the levels of cyclin D1 at the G2/M phase of cell cycle are crucial for the cells to decide if they can undergo another round of replication. Cyclin D1 nuclear import plays a very critical role in cardiomyocyte proliferation (Tamamori-Adachi et al., 2003). Levels of YB-1 also play a role in deciding if the cell has to undergo further replication, as inferred from the heterozygous mutant DT40 cells (Swamynathan et al. 2002) which showed 4n, 6n and 8n DNA content suggesting that the cells undergo DNA replication but not cell division (replicative senescence). YB-1 is shown to be involved in apoptosis (Swamynathan et al., 2002; Schittek et al., 2007). Data from the three YB-1 N-terminal protein internalization analysis also implicates YB-1 in apoptosis. Similarly cyclin D1 is also implicated in cell apoptosis (Hiyama et al., 1999; Duquesne et al., 2001).

The objectives of the current study are to determine if YB-1 interacts with any of the cyclins at the protein level and if so, to initiate a preliminary investigation to understand the details of this interaction. 


\subsection{Materials and methods}

\subsubsection{Antibodies and reagents}

All the chemical reagents, unless otherwise specified were purchased from SigmaAldrich (St. Louis, MO). The Minimum essential medium (cat \# 11095-080), Dulbecco's modified medium (cat \# 1965-092), Penicillin-Streptomycin (cat \# 15140-148) and Trypsin-EDTA (cat \# 25300) was obtained from Invitrogen-Gibco (Carlsbad, CA). The YB-1 polyclonal antibody $(10 \mathrm{mg} / \mathrm{ml})$ was generated for our lab by Sigma. The antibodies against all the cyclins (cat \# RB212-P1, RB-008-P1, RB-1548-P1 and MS-221-P1, $1 \mathrm{mg} / \mathrm{ml}$ ) were obtained from Labvision (Fremont, CA). The antibody against GFP (cat \# A11122, $2 \mathrm{mg} / \mathrm{ml}$ ) and the fluorescent-labeled secondary antibodies (cat \#s A21429 and A11029, 2mg/ml) were from Invitrogen-Molecular probes (Carlsbad, CA). The lamin antibody (cat \# 612162, $250 \mu \mathrm{g} / \mathrm{ml}$ ) was from BD Biosciences (San Jose, CA). The chemiluminescence western blot developing solution kit (cat \# 34080) and the BCA protein assay kit (cat \# 23225) was from Pierce (Rockford, IL). The Vectashield Mounting Medium containing DAPI (cat \# H-1200) was from Vector laboratories (Burlingame, CA). The protein A Sepharose beads (cat \# 17-0780-01) were from Amersham Biosciences (Piscataway, NJ).

\subsubsection{Cell culture}

Rat Hepatoma cells (ATCC-H-411E) were grown in monolayers in Minimum Essential medium supplemented with 10\% FBS, 100U/ml Penicillin, $100 \mu \mathrm{g} / \mathrm{ml}$ Streptomycin and 5\% $\mathrm{CO}_{2}: 95 \%$ air, in a humidified incubator maintained at $37^{\circ} \mathrm{C}$. Cells were subcultured 1:5 at confluency with $0.05 \%$ trypsin-EDTA. Cell synchronization at G1/S phase was done using a double thymidine block with $2 \mathrm{mM}$ thymidine as described previously (Smits et al., 2000). G2/M phase synchronization was done with $2.5 \mu \mathrm{g} / \mathrm{ml}$ nocodazole for $24 \mathrm{hrs}$. The cell synchronizations were confirmed by FACS analysis. Inhibition of YB-1 phosphorylation was done by incubating cells with $0.4 \mu \mathrm{M}$ wortmannin for $24 \mathrm{hrs}$ as described previously (Evdokimova et al., 2006). Inhibition of the cyclin D1 phosphorylation was done by incubating cells with $20 \mathrm{mM} \mathrm{LiCl}$ for $24 \mathrm{hrs}$. as described previously (Diehl et al., 1998). Transcriptional block was provided by incubating cells with $2 \mu \mathrm{g} / \mathrm{ml}$ actinomycin for time periods ranging from $30 \mathrm{~min}$. to 24 hrs.

\subsubsection{FACS analysis}

For confirmation of successful synchronization and cell cycle analysis, synchronized cells were harvested by trypsinization or scraping on ice and pelleted down at $500 \mathrm{~g}$ for $5 \mathrm{~min}$. The pellets were washed with PBS, cells were fixed with ice cold $70 \%$ $\mathrm{EtOH}$, incubated on ice for $45 \mathrm{~min}$. and pelleted down at $500 \mathrm{~g}$ for $5 \mathrm{~min}$. The pellets were washed with Hanks Balanced Salt Solution (HBSS) containing 1\% BSA. The 
resulting pellets were resuspended in Propidium Iodide buffer ( $1: 1$ dilution of $20 \mathrm{ug} / \mathrm{ml}$ of PI in HBSS and $1 \mathrm{mg} / \mathrm{ml}$ RNAse A) and incubated for $30 \mathrm{~min}$. in a $37^{\circ} \mathrm{C}$ water bath. Samples were analyzed using a Becton Dickinson FACS Calibur.

\subsubsection{Immunoprecipitation and immunoblotting}

After trypsinization, harvested cells were centrifuged at $500 \mathrm{~g}$. The pellets were washed with ice cold PBS, and processed either for whole cell extract preparation or nuclear and cytoplasmic extracts preparation. For whole cell extract preparation, the pellets were lysed in Ripa buffer (10 mM Tris-HCl pH-7.4, 5mM EDTA, $150 \mathrm{mM} \mathrm{NaCl}$, $1 \%$ sodium deoxycholate, $0.1 \%$ SDS, $1 \%$ Triton X-100), supplemented with freshly added protease inhibitors ( $1 \mu \mathrm{g} / \mathrm{ml}$ leupeptin, $1 \mu \mathrm{g} / \mathrm{ml}$ aprotinin and $1 \mathrm{mM}$ PMSF). Samples were kept on ice for $5 \mathrm{~min}$. and then sonicated at Branson 4 settings for 8 cycles of $15 \mathrm{sec}$. each with intermittent chilling on ice. The lysates were then stored on ice for $30 \mathrm{~min}$., clarified by high-speed centrifugation at $8000 \mathrm{~g}$ for $5 \mathrm{~min}$. at $4{ }^{\circ} \mathrm{C}$ and the supernatant was used as whole cell extracts. For nuclear and cytoplasmic fractionation, the cell pellets were resuspended in Buffer A (50 mM NaCl, $10 \mathrm{mM}$ HEPES pH-8.0, 500 $\mathrm{mM}$ sucrose, $1 \mathrm{mM}$ EDTA, $0.2 \%$ Triton-X-100, freshly added protease inhibitors mentioned above and $7 \mathrm{mM} \beta$-Mercaptoethanol), vortexed at high speed for $45 \mathrm{sec}$., spun down at $2000 \mathrm{~g}$ for $2 \mathrm{~min}$. at $4{ }^{\circ} \mathrm{C}$ and the supernatant was stored as cytoplasmic extract. The pellets were resuspended gently in Buffer B $(50 \mathrm{mM} \mathrm{NaCl}, 10 \mathrm{mM}$ HEPES pH-8.0, 25\% glycerol, $0.1 \mathrm{mM}$ EDTA, protease inhibitors and $7 \mathrm{mM} \beta$-Mercaptoethanol), spun down at $2000 \mathrm{~g}$ for $2 \mathrm{~min}$. at $4^{\circ} \mathrm{C}$ and the supernatant was discarded. Resulting pellets were resuspended in Buffer C (350 mM NaCl, 10 mM HEPES pH-8.0, 25\% glycerol, 0.1 $\mathrm{mM}$ EDTA, protease inhibitors and $7 \mathrm{mM} \beta$-Mercaptoethanol) and incubated on ice for $30 \mathrm{~min}$. with intermittent high-speed vortexing, spun down at $11000 \mathrm{~g}$ for $15 \mathrm{~min}$ at $4{ }^{\circ} \mathrm{C}$. The resulting supernatant was stored as nuclear extract. All samples were stored at $-80^{\circ} \mathrm{C}$ until use. $200 \mu \mathrm{l}$ aliquots of either the whole cell extracts or the nuclear and cytoplasmic extracts were diluted to $500 \mu \mathrm{l}$ in appropriate buffers and used for immunoprecipitation. In short equivalent amounts of whole cell lysates or nuclear and cytoplasmic extracts were incubated with 1:1000 dilution of YB-1/cyclin D1 antibody or 1:2000 dilution of GFP antibody overnight at $4^{\circ} \mathrm{C}$ on a rocker. The next day equal amount of Protein $\mathrm{A}$ Sepharose beads were added to each sample and incubated for $2 \mathrm{hrs}$. at room temperature on a rocker. The beads were pelleted down at $8000 \mathrm{~g}$ for $4 \mathrm{~min}$. and the pellet was washed with lysis buffer twice. The resulting pellet was resuspended in equal amounts of sample buffer and loaded onto 12\% SDS-PAGE gels. Primary antibody at the dilution of 1:2000 for YB-1/cyclin D1/lamin and 1:7500 dilution for GFP was used for incubation of blots overnight at $4{ }^{\circ} \mathrm{C}$ and secondary antibody at 1:5000 dilution for YB-1/cyclin D1/lamin and 1:15000 dilution for GFP for $1 \mathrm{hr}$. at room temperature. The blot was developed using chemiluminescence (SuperSignal West Pico Chemiluminescent Substrate) according to the manufacturers protocol. 


\subsubsection{Quantitation of immunoblotting data}

All images were scanned and exported as TIFF files to Adobe Photoshop software (San Hose, CA) for subsequent analysis. Each image was adjusted to invert the scale. A box was drawn around the biggest band using Marquee tool and histogram analysis was done. The mean was noted down and then the same box was used to drag down to another band to repeat the process. For each band, representative integrated density values were computed after subtracting the background density. This gave the exact intensity of each band. Mean integrated density values from three independent experiments were calculated and these values were used to draw graphs using Microsoft Excel programming. Statistical analysis of the mean intensity data was performed using the Student's t test using the GraphPad software and results were considered statistically significant if the $\mathrm{P}$ value of $<0.002$ was reached.

\subsubsection{Immunocytochemistry and indirect immunofluorescence}

For immunocytochemistry, cells were plated on cover slips. After each time point, cells were rinsed twice with PBS, fixed with $4 \%$ p-formaldehyde for $15 \mathrm{~min}$., washed with PBS twice and stored at $4^{\circ} \mathrm{C}$, until all time point samples had been collected. The cells were blocked with 5\% goat serum and 3\% BSA in PBS for 30 min. and rinsed with PBS thrice. Cells were then incubated with primary antibodies (Mouse-YB-1 (1:300), Rabbit Cyclin D1 (1:500), Rabbit pSER (1:500) and Rabbit Lamin (1:500)) for 3 hrs. and rinsed with PBS thrice. Then the cells were incubated with secondary antibodies - FITC conjugated Anti-Rabbit IgG (1:500) and Texas-Red conjugated Anti-Mouse IgG (1:500) for $1 \mathrm{hr}$. in the absence of light. The coverslips were rinsed with PBS thrice, once with water to remove any salts and left to air-dry for $45 \mathrm{~min}$. in absence of light. The coverslips were mounted onto glass slides using Vectashield Mounting Medium containing DAPI to counter stain nuclei. The slides were stored at $-20^{\circ} \mathrm{C}$ until analyzed by confocal microscopy.

\subsubsection{Confocal microscopy}

Confocal images were captured using LSM 510 confocal laser-scanning three-color microscope (Carl Zeiss, Jena, Germany). Images were captured using the Argon laser at $75 \%$ output and $\mathrm{HeNe}$ laser at excitation wavelengths $488 \mathrm{~nm}$ and $543 \mathrm{~nm}$ respectively. Optical slice thickness was set to $0.3 \mu \mathrm{m}$, magnification to $100 \mathrm{x}$ and sections presented were taken approximately at mid height level of cells. Photomultiplier gain and laser power were identical within each experiment. A minimum of 100 stained cells were scored for each experiment reported. The images were analyzed and adjusted for contrast in Adobe Photoshop 5.0 (San Jose, CA). 


\subsubsection{Quantitation of immunofluorescence data}

Each stack of confocal images were imported and opened on the LSM image scanner. The histogram analysis was performed on atleast 4 cells from each slice and atleast 5 slices for each experiment and all the slices were analyzed for the same cells. To start with, the outlining tool was used to demarcate each cell area. The brightness contrast feature was used to minimize one colour and the total pixel count was noted down for the other colour. Then the first colour was restored back to the default setting and the colocalization coefficient for channel one was noted down. This gave the $\%$ of colocalized pixels of one colour with the other. The total number of colocalized pixels were then calculated. Averages of the total pixels and the colocalized pixels were determined. The average $\%$ of total colocalized pixels was then calculated by comparison of the average of total pixels vs. the average of colocalized pixels. This gave us the $\%$ co-localization of the first protein to the second protein. Similarly the \% co-localization of the second protein with the first was also calculated.

\subsection{Results}

\subsubsection{Interaction of YB-1 with Cyclin D1}

Earlier studies show that YB-1 regulates transcription of two cyclins i.e. cyclins A and B1 (Jurchott et al., 2003). YB-1 was also shown to translationally regulate cyclin D1 mRNA (Evdokimova et al., 2006). To investigate if YB-1 shows an interaction with any of the cyclins at a protein level, we performed co-immunoprecipitation assays on whole cell extracts of exponentially growing rat hepatoma cells with antibodies against various cyclins (A, B1, D1 and D2) and YB-1 and YB-2 respectively. Equal amounts of immunoprecipitated samples were blotted on a $12 \%$ SDS-PAGE denaturing gel and probed for presence of YB-1 or the respective cyclins. We detected a signal for YB-1 in the cyclin D1 immunoprecipitated sample (Fig. 3.1) but not in samples immunoprecipitated with the three other cyclin antibodies. YB-1 immunoprecipitated sample was used as a positive control and YB-2 immunoprecipitated sample as an experimental negative control. Cell lysates incubated with the beads in absence of any antibodies were used as an internal negative control, to rule out any non-specific signals. Reciprocal blots by immunoprecipitation with YB-1 antibody and probing for the abovementioned cyclins confirmed immunoprecipitation of YB-1 only with cyclin D1 and vice versa (data not shown). The same results were also confirmed in rat aortic smooth muscle (RASM) cells (data not shown). These results indicate that YB-1 protein interacts with cyclin D1.

\subsubsection{YB-1 co-localizes with Cyclin D1 predominantly at the G2/M phase}

YB-1 is predominantly a cytoplasmic protein and only about 5\% of the total YB-1 is localized in the nucleus of exponentially growing cells (Jurchott et al., 2003). Initial 


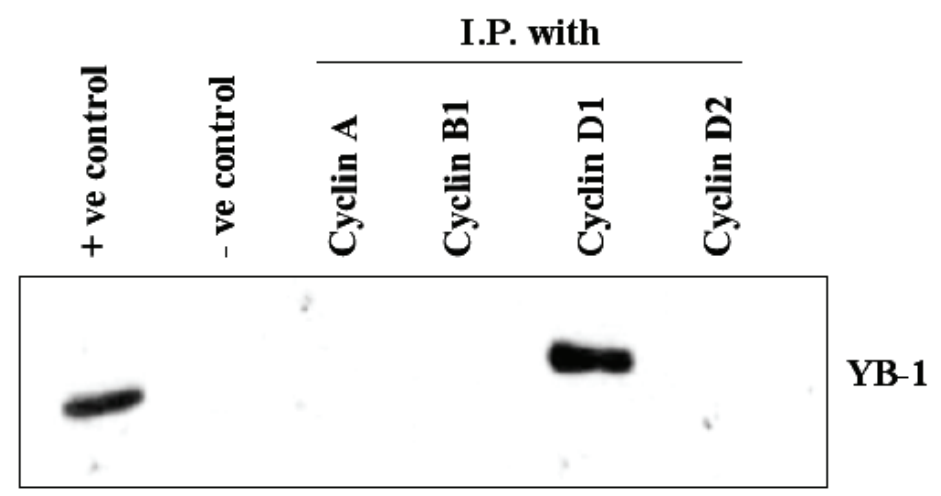

\section{Fig. 3.1 YB-1 and Cyclin D1 co-immunoprecipitate with each other in rat} hepatoma cells

Whole cell extracts of exponentially growing unsupplemented rat hepatoma cells were immunoprecipitated with antibodies against cyclin A, B1, D1 and D2. The resulting samples were analyzed by immunoblotting for the presence of YB-1 using a polyclonal YB-1 antibody. YB-1 signals were detected only with YB-1 antibody (+ve control) and cyclin D1, but not with YB-2 (-ve control) or the other cyclins and vice versa (data not shown), suggesting that TYB-1 interacts with only cyclin D1 among the tested cyclins. This co-immunoprecipitation was also confirmed in Rat aortic smooth muscle cells (data not shown). 
studies indicated that cyclin D1 is localized predominantly in the nucleus of asynchronously growing cells (Baldin et al., 1993). However, later studies showed subcellular localization of cyclin D1 to oscillate during the cell cycle (Lukas et al., 1994; Diehl et al., 1998). Owing to this differential distribution of both proteins in the subcellular compartments through the cell cycle, we proceeded to investigate the relative distribution of YB-1 and cyclin D1 in the nucleus and cytoplasm of cells at different phases of cell cycle. For this purpose, G1/S and G2/M phase synchronization of cells was done. Double thymidine block, which inhibits DNA replication by inhibiting the conversion of CDP to dCDP, was used to render a G1/S phase block as described previously (Smits et al., 2000). G2/M phase synchronization was done using the drug nocodazole, which causes microtubule depolymerization, thus blocking the cells in prometaphase and thereby rendering the G2/M phase block. The \% of synchronized cells was estimated by FACS analysis to ensure that majority of the cell population got synchronized at the respective phases (Fig. 3.2 and 3.3), before any further steps were performed.

Immunocytochemistry and indirect immunofluorescence / co-localization analysis was performed on these synchronized cells. For this purpose, unsynchronized and synchronized cells were fixed onto coverslips and labeled for YB-1 and Cyclin D1 as mentioned previously. The relative localizations of YB-1 and cyclin D1 in the nucleus and cytoplasmic compartments of these cells were detected by confocal microscopy. In the unsynchronized control and G1/S phase synchronized cells, YB-1 was present predominantly in the cytoplasm (Fig. 3.4, panels A and D) whereas cyclin D1 was predominantly in the nucleus (Fig. 3.4, panels $\mathrm{C}$ and F). In both cases minimal colocalization of YB-1 and cyclin D1 was seen in both nucleus and cytoplasm. In stark contrast, the distribution and co-localization pattern of the two proteins in $\mathrm{G} 2 / \mathrm{M}$ phase blocked cells showed a drastic change. Negligible amounts of YB-1 and cyclin D1 were detected in the nucleus of the G2/M phase blocked cells (Fig. 3.4, panels G and I). Most of the YB-1 and cyclin D1 was detected in the cytoplasm (Fig. 3.4, panels G and I) and their co localization was very predominant as evidenced by the yellow dots seen in the overlap of both the images, when compared to the wild type and G1/S phase blocked cells (Fig. 3.4, compare panels B and E with $\mathrm{H}$ ).

In order to confirm this result, co-immunoprecipitation and immunoblot analysis of unsynchronized and G2/M phase synchronized cells was performed. Nuclear, cytoplasmic and nuclear + cytoplasmic fractions were used for the analysis. The nuclear + cytoplasmic fractions were used in lieu of whole cell extracts to investigate if the YB-1 cyclin D1 interaction occurs post-lysis of cells. In case of unsynchronized cells, we detected YB-1 signals mainly in cytoplasmic or nuclear + cytoplasmic samples immunoprecipitated with YB-1 (Fig. 3.5, left). Cyclin D1 was detected mainly in the nuclear and nuclear + cytoplasmic samples, immunoprecipitated by cyclin D1. This suggested that in unsynchronized cells, YB-1 is predominantly present in the cytoplasm, whereas Cyclin D1 is in the nucleus. In the nocodazole treated (G2/M phase blocked) cells, no signal was detected for either YB-1 or Cyclin D1 in the nuclear extracts, confirming our previous result. We detected both YB-1 and cyclin D1 in cytoplasmic and cytoplasmic + nuclear samples immunoprecipitated with either of the antibodies (Fig. 3.5 


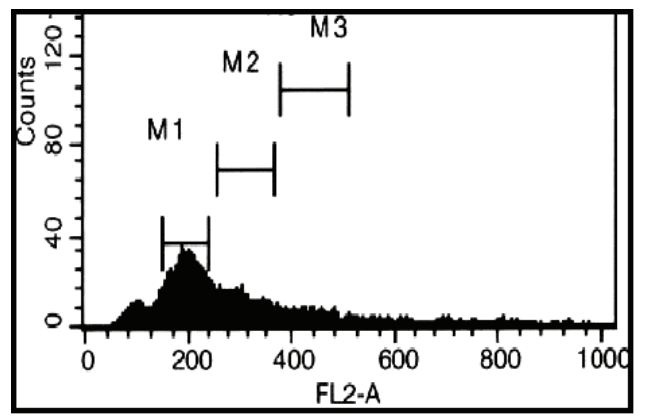

Fig. 3.2 Synchronization of cells at the G1/S phase of cell cycle by a double thymidine block

Rat hepatoma cells were blocked at the G1/S phase of cell cycle by a double thymidine block. FACS analysis performed on the cells confirms that a major proportion of cells are blocked at the G1/S phase of cell cycle.

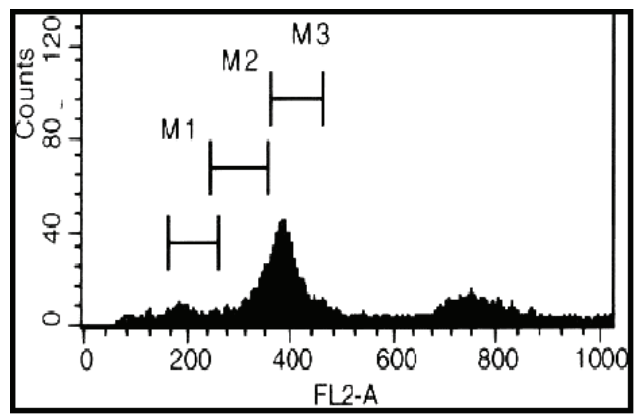

Fig. 3.3 Synchronization of cells at the G2/M phase of cell cycle by a nocodazole block

Rat hepatoma cells were blocked at the G2/M phase of cell cycle by a nocodazole block. FACS analysis performed on the cells confirms that a major proportion of cells are blocked at the G2/M phase of cell cycle. 
Fig. 3.4 Co-localization of YB-1 and Cyclin D1 in G2/M phase

Exponentially growing unsynchronized and G1/S phase synchronized cells with a double thymidine block or $\mathrm{G} 2 / \mathrm{M}$ phase synchronized cells with a nocodazole block grown on coverslips were fixed with 4\% p-formaldehyde and blocked with 3\% goat serum and BSA. The cells were then labeled with primary antibodies against YB-1 and cyclin D1 and secondary antibodies conjugated to FITC or Texas Red respectively. Distribution of YB-1 and cyclin D1 in these cells was analyzed by confocal microscopy. We detected the presence of both YB-1 and cyclin D1 predominantly in the cytoplasm of nocodazoleblocked cells where they co-localized with each other (panels G to I), as evidenced by the yellow dots where FITC and Texas Red overlap. In control (panels A to C) and double thymidine-blocked cells (panels D to F) cyclin D1 was seen in both nucleus and cytoplasm and YB-1 in the cytoplasm with minimal co-localization between the two suggesting that YB-1 colocalization with cyclin D1 is predominant only in the cytoplasm of cells in $\mathrm{G} 2 / \mathrm{M}$ phase of cell cycle. 

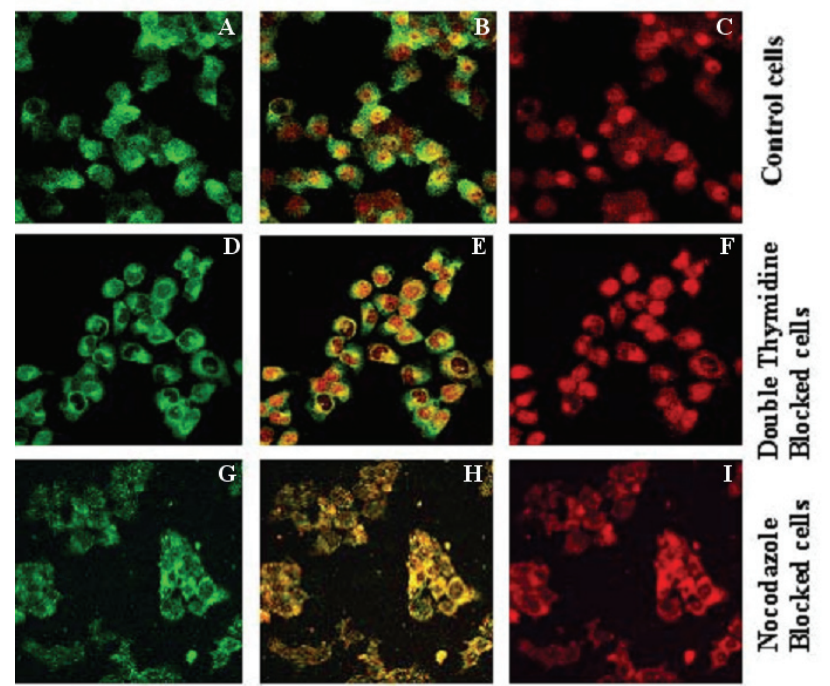

造
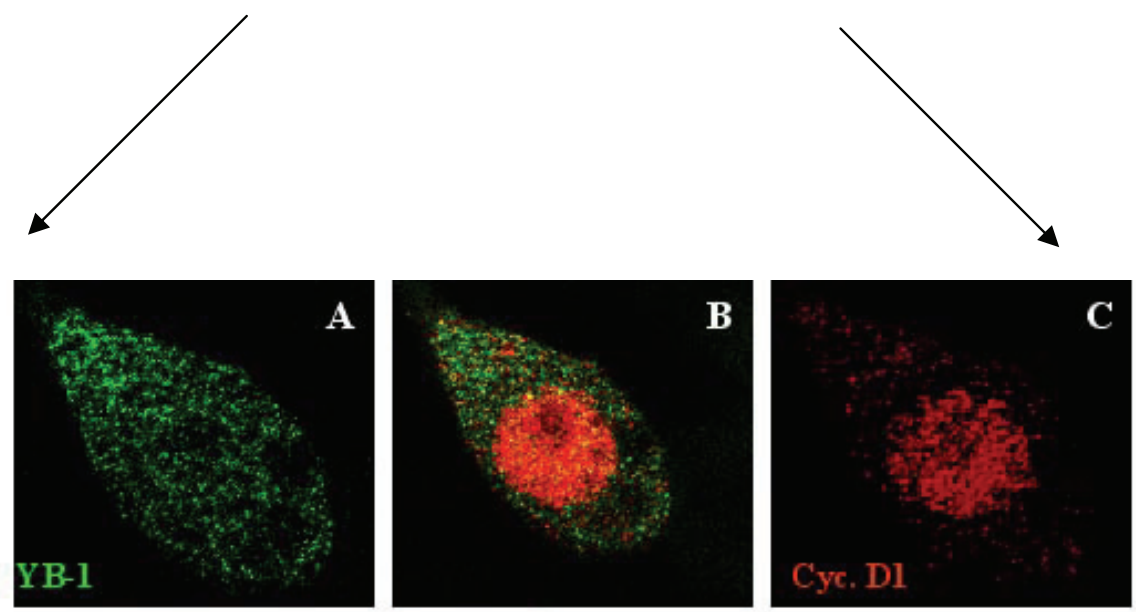

Control

Cells
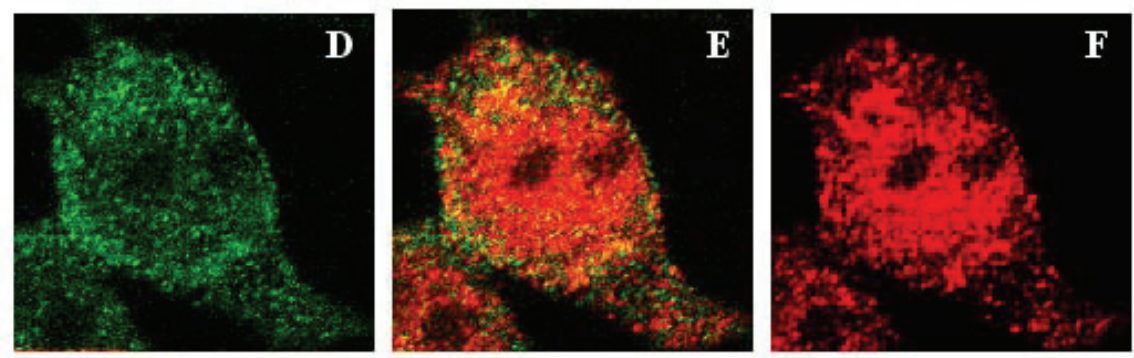

Double

Thymidine Blocked cells
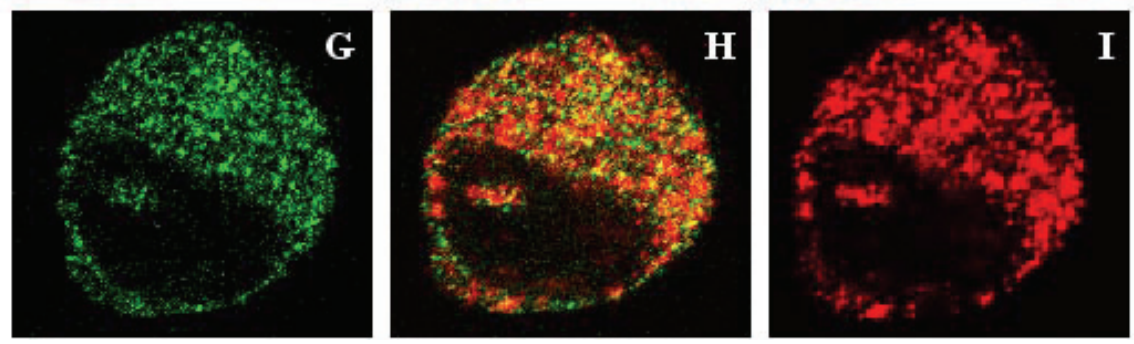

Nocodazole blocked cells 


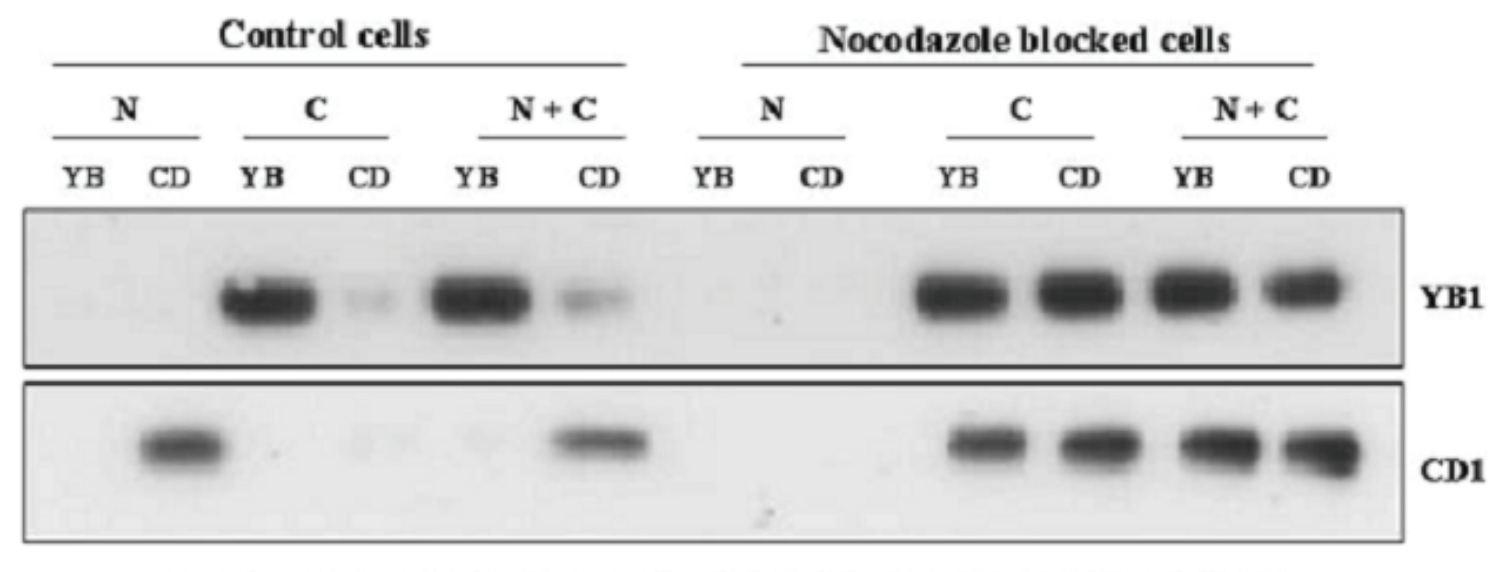

YB $=$ YB-1 $\quad$ CD $=$ Cyclin D1 $N=$ Nuclear extract $\quad C=$ Cytoplasmic extract

Fig. 3.5 Interaction of YB-1 with Cyclin D1 and vice-versa is seen predominantly at the $\mathbf{G} 2 / \mathrm{M}$ phase of cell cycle

Nuclear $(\mathrm{N})$, cytoplasmic $(\mathrm{C})$ and nuclear + cytoplasmic $(\mathrm{N}+\mathrm{C})$ extracts of exponentially growing unsynchronized $\mathrm{G} 2 / \mathrm{M}$ phase synchronized cells were immunoprecipitated with antibodies against YB-1 or cyclin D1 and analyzed by western blotting to investigate the total \% of immunoprecipitation of YB-1 with cyclin D1 and vice versa from each of the above mentioned extracts. A representative blot of immunoprecipitation and immunoblot analysis is hown here. We show that YB-1 immunoprecipitates with Cyclin D1 and vice versa predominantly in the cytoplasmic and nuclear + cytoplasmic extracts of only the G2/M phase blocked cells confirming the results got by immunocytochemistry and immunofluorescence analysis shown in Fig. 3.4. 
right side), confirming the results of immunocytochemistry and co-localization experiments. These results suggest that YB-1 and Cyclin D1 interact and co-localize in the cytoplasm of $\mathrm{G} 2 / \mathrm{M}$ phase blocked cells.

\subsubsection{Quantitative interaction of YB-1 with Cyclin D1 and vice versa}

Having demonstrated the interaction and co-localization of YB-1 and cyclin D1 in the cytoplasm of $\mathrm{G} 2 / \mathrm{M}$ phase blocked cells, we proceeded to measure the quantitative interaction between both the proteins in unsynchronized and $\mathrm{G} 2 / \mathrm{M}$ phase synchronized cells. In order to determine the total amount of pull down of YB-1 with cyclin D1 antibody and vice versa as compared to immunoprecipitation by its own antibody, co-immunoprecipitation and immunoblot analysis were performed as previously. Three independent experiments were performed for statistical analysis. The intensity of the signals was calculated and the mean integrated intensity from three experiments is reported in Fig. 3.6.

As expected, in the control unsynchronized cells, very faint signals for YB-1 in the nuclear extracts and cyclin D1 in the cytoplasmic extracts were detected. About $5-7 \%$ of total YB-1 and cyclin D1 immunoprecipitation was seen with the cyclin D1 and YB-1 antibodies in the nuclear + cytoplasmic fractions (Fig. 3.6). About $80 \%$ of the total YB-1 (total YB-1 = YB-1 immunoprecipitated with its own antibody, which is normalized to read 100\%) immunoprecipitated with Cyclin D1 antibody and vice versa in the cytoplasmic and cytoplasmic + nuclear fractions of the G2/M phase synchronized cells (Fig. 3.6). In contrast negligible amounts of either protein were detected in the nuclear fractions. Using the student's t-test the $\mathrm{p}$ value for these calculations was determined to be $<0.0019$, which is considered statistically significant.

We then quantitated the \% co-localization of YB-1 and cyclin D1 in control and nocodazole treated cells from the immunofluorescence confocal images as mentioned in the methods. The results obtained are very similar to the immunoblotting quantitation data (Tables 3.1 and 3.2). This reinforces our previous conclusion that YB-1 and cyclin D1 interaction is predominant in the cytoplasm of cells in the G2/M phase of cell cycle.

\subsubsection{N-terminus of YB-1 is involved in the interaction of $Y B-1$ with Cyclin D1}

After demonstrating the interaction of cyclin D1 with full length YB-1 protein, we proceeded to investigate the involvement of the N-terminal domain of YB-1 in this interaction. Most proteins are composed of multiple modular interacting domains. Typically, protein interaction domains are independently folding modules of a short stretch of amino acids, which can be expressed in isolation from host proteins while retaining their intrinsic ability to bind their physiological partners. From our previous study (as shown in chapter 2), we had three YB-1 N-terminal proteins, APYB26GFP, APYB36GFP and APYB77GFP, each containing a short stretch of amino acids in fusion 


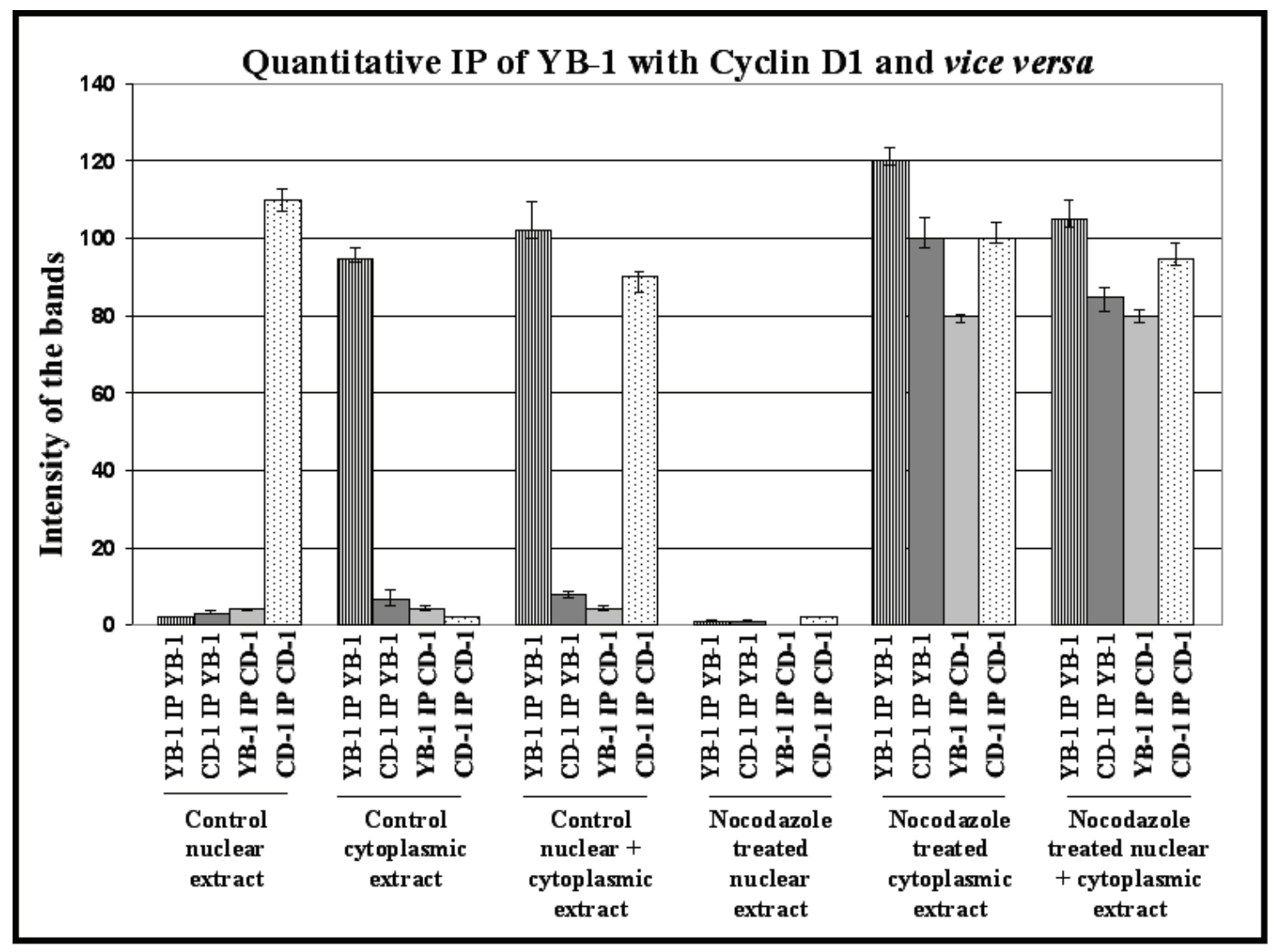

Fig. 3.6 YB-1 immunoprecipitates approximately $80 \%$ of the total Cyclin D1 and vice versa at the $\mathrm{G} 2 / \mathrm{M}$ phase of cell cycle

The mean integrated intensity of the blot signals from three independent experiments of co-immunoprecipitation and immunoblot analysis of unsynchronized and G2/M phase synchronized cell's nuclear and cytoplasmic extracts shows that approximately $80 \%$ of the total YB-1 got immunoprecipitated with cyclin D1 antibody and vice versa in the cytoplasmic and cytoplasmic + nuclear fractions of the G2/M phase synchronized cells. Faint signals were seen in the nuclear extracts or in unsynchronized cell extracts, suggesting that YB-1 cyclin D1 interaction is predominant in the cytoplasm of G2/M phase cells. 
Table 3.1 Quantitative co-localization of YB-1 with Cyclin D1 and vice versa in control cells

\begin{tabular}{ccc}
$\begin{array}{c}\text { Cell } \\
\#\end{array}$ & (FITC Y overlapping Texas Red) & (Texas Red overlapping FITC) \\
\hline 1 & 7.95 & 8.10 \\
2 & 7.22 & 8.21 \\
3 & 8.90 & 8.07 \\
4 & 6.41 & 5.85 \\
\hline
\end{tabular}

We detected minimal co-localization of YB-1 with cyclin D1 and vice versa in control cells by quantitation of the immunocytochemistry data.

Table 3.2 Quantitative co-localization of YB-1 with Cyclin D1 and vice versa in nocodazole blocked cells

\begin{tabular}{ccc}
$\begin{array}{c}\text { Cell } \\
\#\end{array}$ & (FITC overlapping Texas Red) & (Texas Red overlapping FITC) \\
\hline 1 & 69.37 & 76.46 \\
2 & 74.52 & 79.74 \\
3 & 79.28 & 83.29 \\
4 & 79.44 & 81.95 \\
\hline
\end{tabular}

A very high \% of co-localization of YB-1 with cyclin D1 and vice versa was detected in nocodazole blocked $\mathrm{G} 2 / \mathrm{M}$ phase cells by quantitation of the immunocytochemistry data. 
with an antennapedia and GFP sequence and a control clone APGFP lacking any YB-1 sequence. In order to determine if the YB-1 N-terminal proteins interact with cyclin D1, rat hepatoma cells were incubated with $40 \mu \mathrm{g} / \mathrm{ml}$ of the four fusion proteins as described previously for 3 and $18 \mathrm{hrs}$. Whole cell extracts of unsynchronized and G2/M phase synchronized cells were incubated with GFP antibody for immunoprecipitation of the internalized fusion proteins and probed with cyclin D1 antibody for analysis of any cyclin D1 immunoprecipitated along with the fusion proteins. As a positive control, cyclin D1 immunoprecipitated with YB-1 antibody was used on the same blot. APGFP, which does not have any YB-1 sequence, acted as our negative control. In unsynchronized cells cyclin D1 band was not detected with GFP in any of the APYBGFP or APGFP incubated cells (Fig. 3.7). However, in nocodazole-blocked cells, cyclin D1 band was detected at both $3 \mathrm{hrs}$. and $18 \mathrm{hrs}$. with APYB26GFP and APYB77GFP incubated cells (Fig. 3.7). This suggests that YB-1 N-terminal sequence is involved in its interaction with cyclin D1 in cells at the G2/M phase of cell cycle. The cyclin D1 signal however was not detected in case of APYB36GFP incubated cells, suggesting that the YB-1 N-terminal sequence present in this clone is either not sufficient or not accessible to interact with cyclin D1. These experiments suggest that the proline rich YB-1 N-terminal sequence is sufficient for immunoprecipitation of cyclin D1 in G2/M phase synchronized rat hepatoma cells.

\subsubsection{Inhibition of $Y B-1$ phosphorylation by Wortmannin results in re-localization of Cyclin D1 in the nucleus even after nocodazole block}

Dynamic protein-protein interactions are frequently regulated by post-translational modifications, most notably phosphorylation. Eukaryotic cells widely use reversible phosphorylation to regulate protein-protein interactions to transmit and integrate signals received from their environment (Pawson, 2004). YB-1 is reported to be phosphorylated mainly by the PI3K-Akt pathway (Sutherland et al. 2005). Wortmannin acts as a pharmacological inhibitor of the PI3K cycle (Evdokimova et al., 2006). In order to investigate if the predominant presence of cyclin D1 in cytoplasm after nocodazole blocking is dependent on the phosphorylation state of YB-1, cells grown on coverslips were incubated with nocodazole and wortmannin simultaneously for $24 \mathrm{hrs}$. and analyzed. We detected a significant amount of cyclin D1 in the nucleus after simultaneous treatments with nocodazole and wortmannin (Fig. 3.8, panel L). YB-1 was detected only in the cytoplasm, especially in the perinuclear space after incubation with only nocodazole or both nocodazole and wortmannin (Fig. 3.8, panels D and J). Wortmannin treatment alone did not result in any change in the pattern of distribution of either YB-1 or cyclin D1 (Fig. 3.8, Panels G and I) as compared to the control cells (Fig. 3.8, panels A to C). Therefore the presence of cyclin D1 in the nucleus can be attributed to incubation with both nocodazole and wortmannin. These results were reproduced and confirmed by western blotting (data not shown). This led us to hypothesize that translocation of cyclin D1 from the nucleus to cytoplasm in the G2/M phase of cells may be dependent on the phosphorylation state of YB-1. 


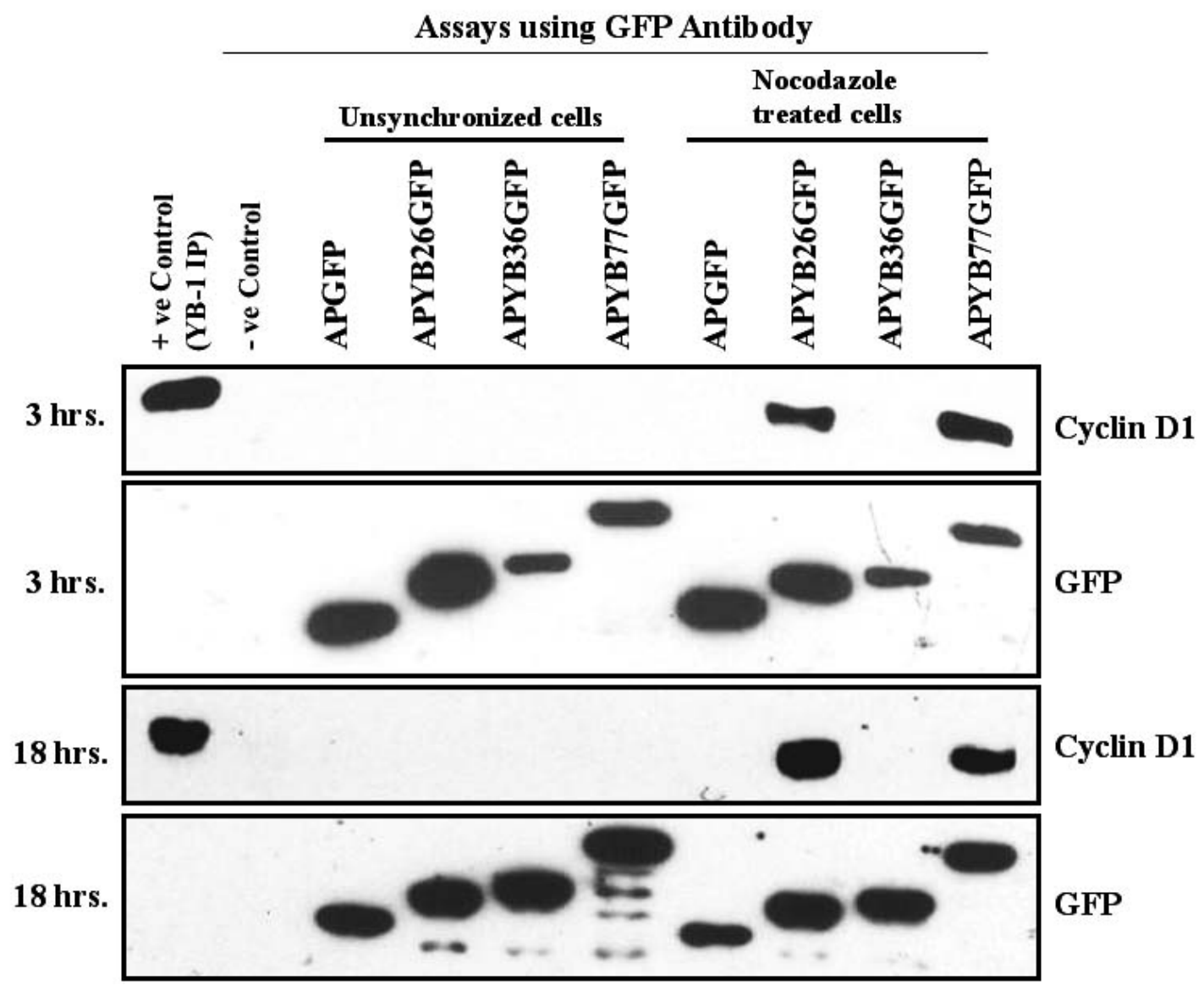

Fig. 3.7 APYB26GFP and APYB77GFP immunoprecipitate Cyclin D1 in G2/M phase blocked rat hepatoma cells

Whole cell lysates of unsynchronized and G2/M phase synchronized cells, incubated with $40 \mu \mathrm{g} / \mathrm{ml}$ of the three APYBGFP and APGFP proteins for 3 and $18 \mathrm{hrs}$. were immunoprecipitated with GFP antibody and resulting samples were analyzed for the presence of cyclin D1 and GFP with their specific antibodies. Immunoprecipitation of cyclin D1 from nocodazole blocked cells with YB-1 antibody was used as a +ve control for cyclin D1 and immunoprecipitation of unincubated cells with GFP antibody was used as a-ve control for both GFP and cyclin D1. Cyclin D1 band was not detected in any samples from unsynchronized cells. We detected cyclin D1 signals in lysates from G2/M phase synchronized cells incubated with APYB26GFP and APYB77GFP proteins, but not with APYB36GFP and APGFP, indicating that the 26 amino acid proline rich sequence from YB-1 N-terminus is necessary and enough for binding with cyclin D1 at the $\mathrm{G} 2 / \mathrm{M}$ phase of cell cycle in rat hepatoma cells. 


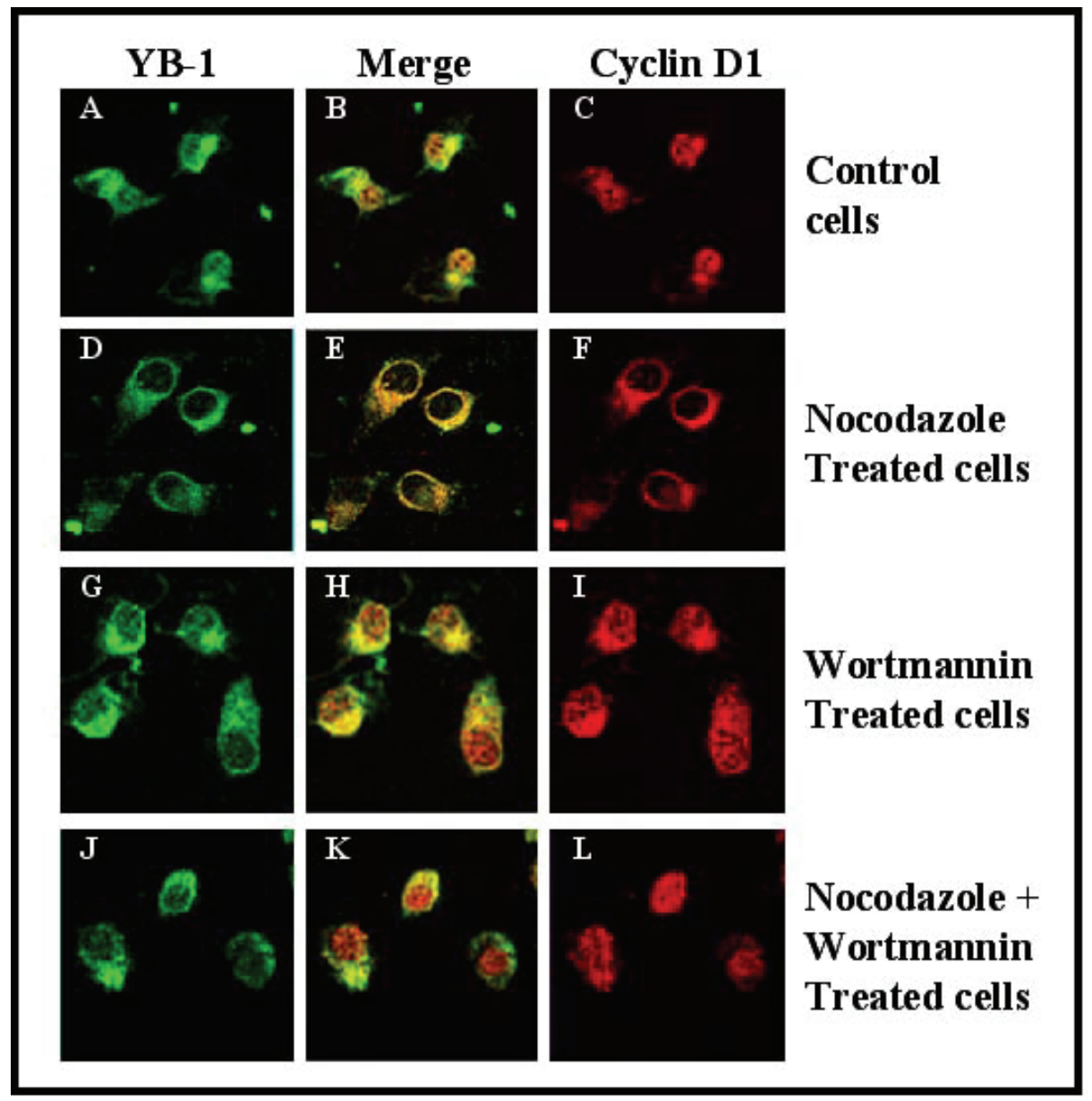

Fig. 3.8 Inhibition of YB-1 phosphorylation by Wortmannin resulted in relocalization of Cyclin D1 in the nucleus even after nocodazole block

Exponentially growing cells on coverslips were incubated with wortmannin and/or nocodazole for $24 \mathrm{hrs}$. and analyzed by immunocytochemistry and indirect immunofluorescence with antibodies against YB-1 and cyclin D1 to investigate the effects of wortmannin incubation on the co-localization of the two proteins in unsynchronized and G2/M phase synchronized cells. Cells treated with only wortmannin showed cyclin D1 in both nucleus and cytoplasm (panel I) and YB-1 in the cytoplasm (panel G) with minimal co-localization (panel H), similar to the distribution of both the proteins in control cells (panels A to C). Nocodazole treated cells showed both proteins colocalized together in the cytoplasm (panels D to F). In contrast to this, treatment of cells with both nocodazole and wortmannin resulted in the presence of cyclin D1 in the nucleus as well as cytoplasm (panel L), whereas YB-1 was seen predominantly in the cytoplasm (panel J). 


\subsubsection{Inhibition of Cyclin D1 phosphorylation does not change co-localization of YB-1 with Cyclin D1}

Cyclin D1 phosphorylation and nuclear export is mediated by glycogen synthase kinase - $3 \beta$ (GSK-3 $\beta$ ) association with nuclear exportin CRM1 (Diehl et al. 1998). To study if inhibition of phosphorylation of cyclin D1 results in a change in co-localization of YB-1 and cyclin D1, we incubated cells with LiCl, which is an inhibitor of GSK-3 $\beta$ activity. $\mathrm{LiCl}$ was previously found to be particularly efficient for this purpose in rat hepatoma cells (Lewitt et al., 2001). In unsynchronized and nocodazole blocked cells, YB-1 and cyclin D1 show similar distribution as described previously (Fig. 3.9, panels A to F). We did not detect any changes in the pattern of distribution or co-localization of YB-1 and cyclin D1 in the $\mathrm{LiCl}$ and $\mathrm{LiCl}+$ nocodazole treated cells (Fig. 3.9, panels $\mathrm{G}$ to $\mathrm{L}$ ), indicating that cyclin D1 phosphorylation states might not determine its interaction or co-localization with YB-1. These results were reproduced and confirmed by western blotting (Fig. 3.10). We conclude that the phosphorylation state of cyclin D1 might not be important for its co-localization with YB-1 in the cytoplasm of cells in G2/M ohase of cell cycle.

To rule out the possibility of cytoplasmic predominance of both proteins due to the breakdown of nuclear membrane, towards initiation of metaphase (the stage at which nocodazole blocks cell cycle), we checked the integrity of the nuclear membrane by immunoblotting and indirect immunofluorescence analysis of lamin, which is integral to the nuclear membrane. The results clearly show lamin only in the nuclear extracts (Fig. 3.11) and the pattern of staining of lamin in both control and G2/M phase blocked cells is similar (Fig. 3.12). This suggests that the predominant co-localization of YB-1 and cyclin D1 in the cytoplasm of G2/M phase blocked cells is most likely due to an active transport of proteins and not just passive diffusion.

\subsubsection{YB-1 - Cyclin D1 interaction is not completely mediated by RNA}

YB-1 forms an important part of ribonucleoproteins (Skabkin et al., 2004). It is involved in translational repression of Cyclin D1 by binding to its mRNA (Evdokimova et al., 2006). To determine if the interaction seen between YB-1 and cyclin D1 is RNA dependent, we treated rat hepatoma whole cell lysates with $100 \mu \mathrm{g} / \mathrm{ml}$ RNAse A for 2 hrs. prior to immunoprecipitation and then probed for the presence of YB-1 and cyclin D1. We detected immunoprecipitation of YB-1 with cyclin D1 and vice versa in the RNAse treated samples as well as untreated samples (Fig. 3.13). We therefore conclude that this YB-1 - cyclin D1 protein-protein interaction is not mediated through RNA.

\subsubsection{YB-1 and Cyclin D1 show a change in distribution pattern after transcriptional block}

YB-1 acts as a transcriptional activator of many genes. Cyclin D1 promoter shows binding sites for AP-1 (Watanabe et al., 1996; Lee et al., 1999) and NF-kB (Guttridge et 


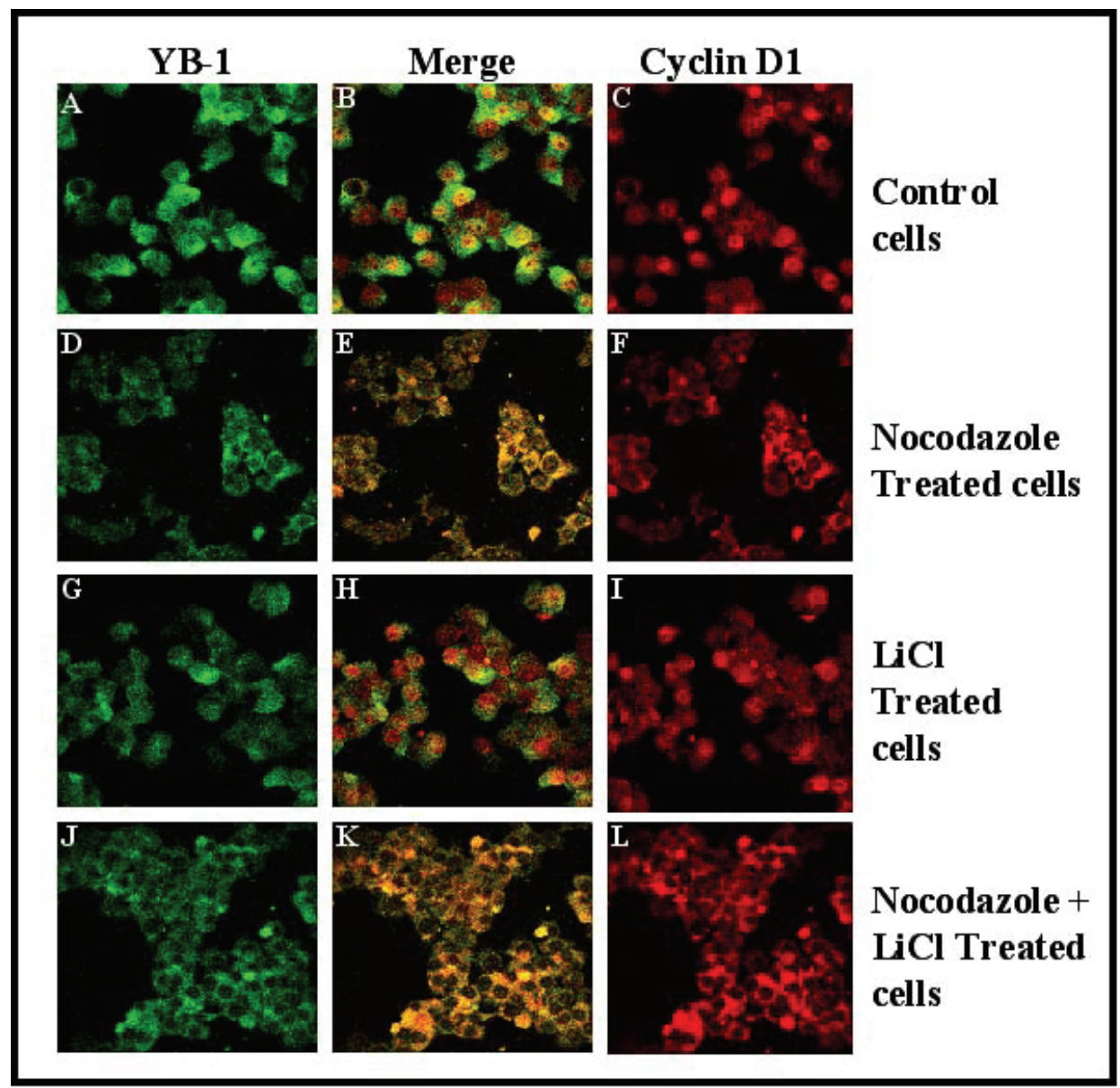

Fig. 3.9 Inhibition of Cyclin D1 phosphorylation with LiCl did not change the colocalization of YB-1 and Cyclin D1 in G2/M phase blocked cells

Exponentially growing cells on coverslips were incubated with $\mathrm{LiCl}$ and/or nocodazole for $24 \mathrm{hrs}$. and analyzed by immunocytochemistry and indirect immunofluorescence with antibodies against YB-1 and cyclin D1 to investigate the effects of $\mathrm{LiCl}$ incubation on the co-localization of the two proteins in unsynchronized and G2/M phase synchronized cells. Cell treated with only $\mathrm{LiCl}$ showed cyclin D1 in both nucleus and cytoplasm (panel I) whereas YB-1 distribution was seen predominantly in the cytoplasm (panel G) with minimal co-localization (panel H), similar to the distribution of both the proteins in control cells (panels A to C). Nocodazole treated cells showed both proteins colocalized together in the cytoplasm (panels $\mathrm{D}$ to F). Treatment of cells with nocodazole and $\mathrm{LiCl}$ also resulted in the presence of both YB-1 and cyclin D1 in the cytoplasm of cells colocalized with each other (panels $\mathrm{J}$ to $\mathrm{L}$ ). 


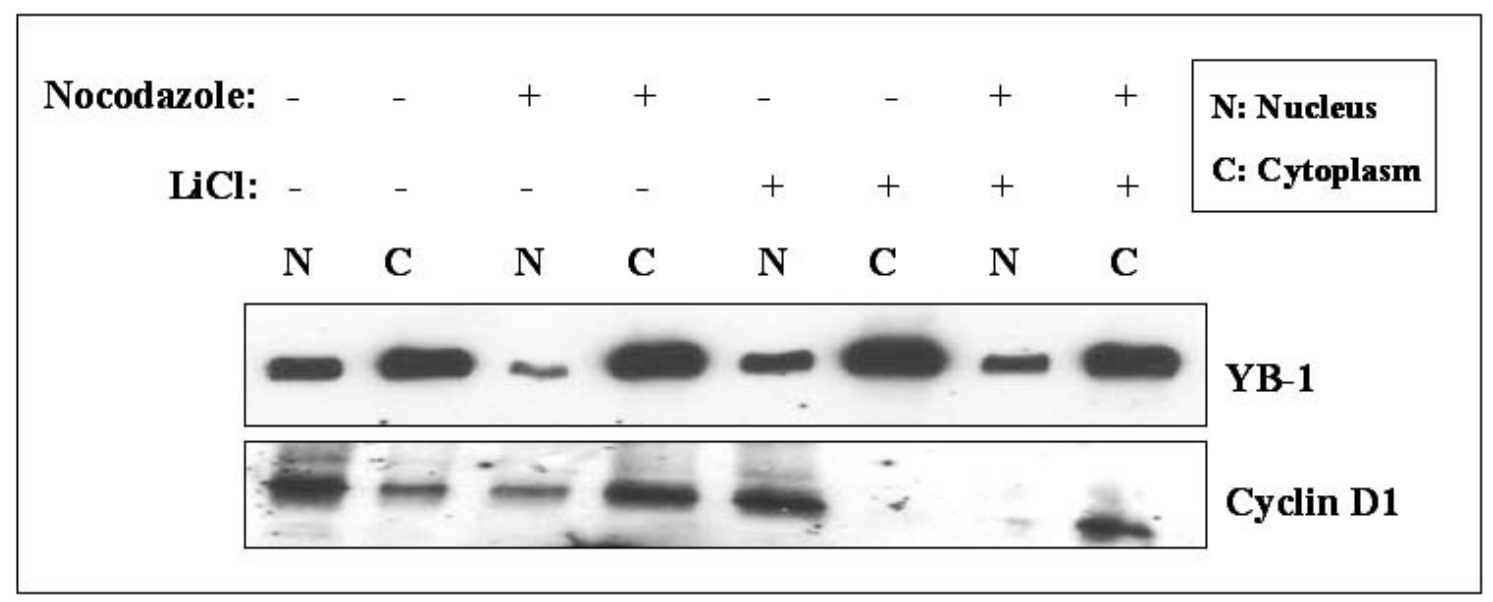

Fig. 3.10 Immunoblot analysis of nuclear and cytoplasmic extracts of cells incubated with $\mathrm{LiCl}$ and/or nocodazole

A representative blot of the nuclear and cytoplasmic extracts of cells treated with either nocodazole and/or LiCl, confirms that the cytoplasmic distribution of cyclin in $\mathrm{G} 2 / \mathrm{M}$ phase cells is independent of its phosphorylation state. Cyclin D1 was present only in the cytoplasmic extracts of nocodazole or nocodazole and $\mathrm{LiCl}$ incubated cells whereas it was mainly seen only in the nuclear extract of $\mathrm{LiCl}$ incubated cells. 


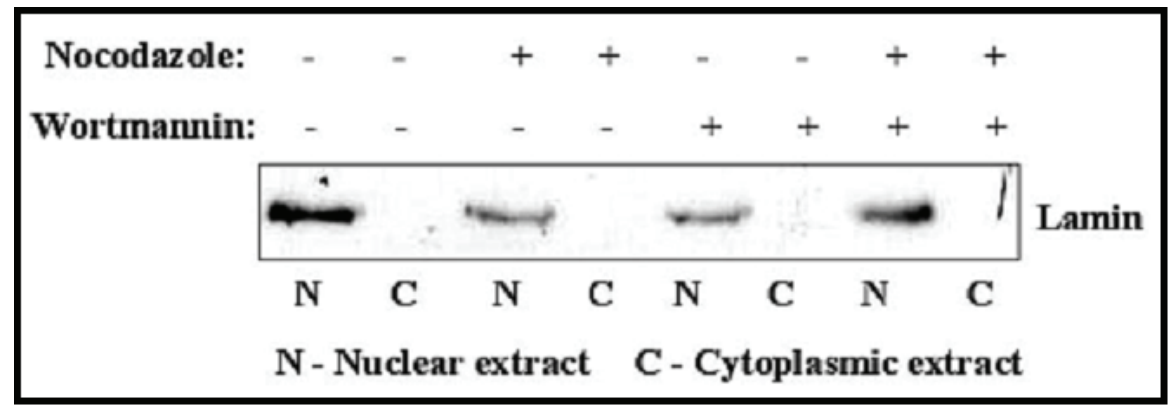

Fig. 3.11 Lamin is seen only in nuclear extracts of cells treated with nocodazole Western blotting analysis of nuclear and cytoplasmic extracts of cells probed with lamin showed the lamin signal only in the nuclear extracts of cells suggesting that the nucleus is intact even after the nocodazole incubation.

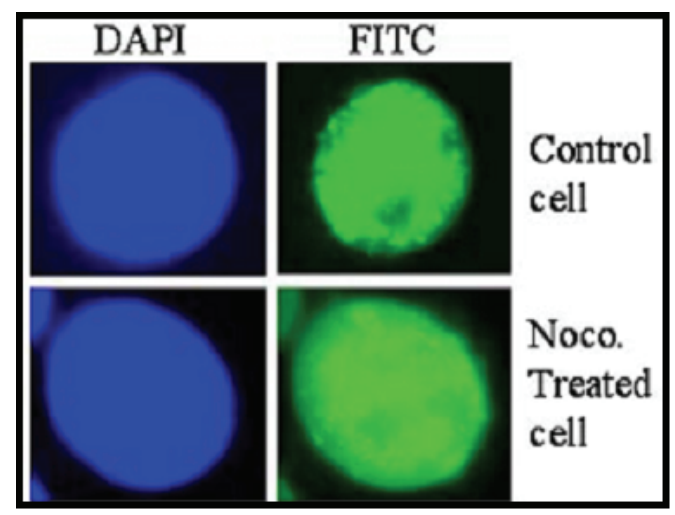

Fig. 3.12 Evidence that nocodazole treatment does not cause breakdown of nuclear membrane

Immunocytochemistry analysis showed similar staining of lamin in both control and nocodazole treated cells, suggesting that the nuclear membrane integrity is maintained in nocodazole treated cells. 


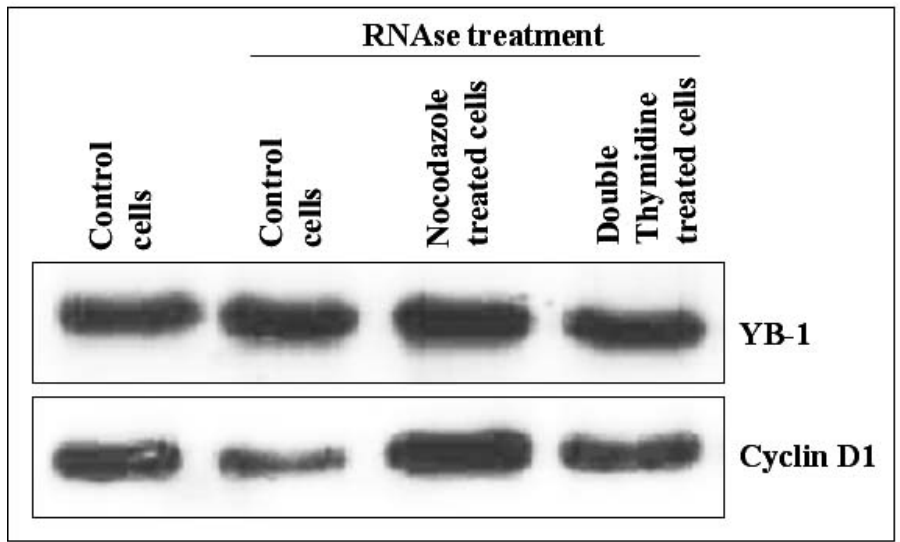

Fig. 3.13 YB-1 and Cyclin D1 interaction is independent of mRNA

Immunoblot analysis of RNase treated whole cell extracts show immunoprecipitation of cyclin D1 with YB-1 indicating the presence of a YB-1 cyclin D1 interaction even in the absence of mRNA. 
al., 1999; Joyce et al., 1999), STAT (Matsumura et al., 1999), E2F/DP (Lee et al., 2000), ATF/CREB (Lee et al., 1999; Nagata et al., 2001) and sp-1/sp-3 (Watanabe et al., 1998; Nagata et al., 2001), but not the inverted CCAAT box repeats to which YB-1 is known to bind. However this just rules out its direct transcriptional regulation by YB-1. However, YB-1 was shown to bind to the NF- $\kappa$ B subunit p65 (Raj et al., 1996) and our preliminary experiments showed an interaction of both YB-1 and cyclin D1 with the NF- $\kappa$ B subunits p52 and Rel B. Therefore, to explore the possibility of indirect transcriptional regulation of cyclin D1 by YB-1, exponentially growing rat hepatoma cells were incubated with actinomycin D for time periods ranging from $30 \mathrm{~min}$. to $24 \mathrm{hrs}$. Immunocytochemistry and indirect immunofluorescence analysis were performed on these cells by YB-1 and cyclin D1 labeling. We noticed a decrease in the levels of nuclear YB-1 and cyclin D1 beginning at the $30 \mathrm{~min}$. time point of incubation (Fig. 3.14, panel B) leading to negligible nuclear and maximal cytoplasmic detection of both proteins at the 1 and $2 \mathrm{hrs}$. time points (Fig. 3.14, panels C and D). Again beginning the 4 hrs. time point and leading to the $8 \mathrm{hrs}$. time point, a small amount of both the proteins were seen in the nucleus (Fig. 3.14, panels $\mathrm{E}$ and F). The $12 \mathrm{hrs}$. and $24 \mathrm{hrs}$. time point slides showed distribution of cyclin D1 in both nucleus and cytoplasm and YB-1 predominantly in the cytoplasm (Fig. 3.14, panels $\mathrm{G}$ and $\mathrm{H}$ ). Since there was no apparent decrease in the total levels of either of the proteins, this experiment could not be used to conclude if YB-1 plays a role in transcriptional regulation of cyclin D1 or not, but we could speculate from the data derived that the relative subcellular distribution of the proteins is inter-related.

\subsection{Discussion}

Y-box binding proteins are involved in many cellular functions. Increased expression of YB-1 has been associated with cell proliferation and transformation (Bargou et al., 1997). Though YB-1 has been shown to be transcriptionally regulating some genes associated with cell division and cell cycle, no study has elucidated the direct mechanism of its regulation of cell proliferation.

Our study for the very first time shows a protein-protein interaction of YB-1 with the cell cycle protein cyclin D1. Cyclin D1 is an important cofactor for several transcription factors (Coqueret, 2002; Ewen et al., 2004). To begin with, in this study, we show co-immunoprecipitation of cyclin D1 with YB-1 and vice versa from crude unsupplemented whole cell lysates. Co-immunoprecipitation is a classic method of detecting protein-protein interactions and has been used in literally thousands of experiments. There are many distinct advantages to analyzing protein-protein interactions by co-immunoprecipitation studies in unsupplemented crude lysates as compared to some other approaches (Phizicky et al., 1995). These include i) its detection of the YB-1 cyclin D1 interaction in midst of all competing proteins present in the crude lysates, ii) both the antigen and the interacting proteins are present in the same relative concentrations found in the cells, iii) elaborate complexes involving YB-1 and cyclin D1 if present, are already in their natural state and can be readily co-precipitated and iv) the two proteins are present in their natural state of posttranslational modifications. Therefore 
Fig. 3.14 YB-1 and Cyclin D1 show a change in distribution after actinomycin D treatment

Indirect immunofluorescence analysis was done on control untreated and actinomycin D treated transcriptionally blocked cells for 0-24 hrs. using antibodies against YB-1 (green) and cyclin D1 (red). YB-1 was detected predominantly in the cytoplasm and cyclin D1 in the nucleus in untreated cells (panel A). Decrease in levels of cyclin D1 was seen starting at 30 min. time point of incubation (panel B) upto the $1 \mathrm{hr}$. time point (panel C) and by 2 hrs. it was seen co-localized with YB-1 predominantly in the cytoplasm (panel D). Cyclin D1 was again detected in the nucleus starting at $4 \mathrm{hrs}$. (panel E) and $8 \mathrm{hrs}$. (panel F) time points. Distribution of cyclin D1 was seen increasing in the nucleus by the $12 \mathrm{hrs}$. and 24 hrs. time periods of incubation (panels $\mathrm{G}$ and $\mathrm{H}$ ). 

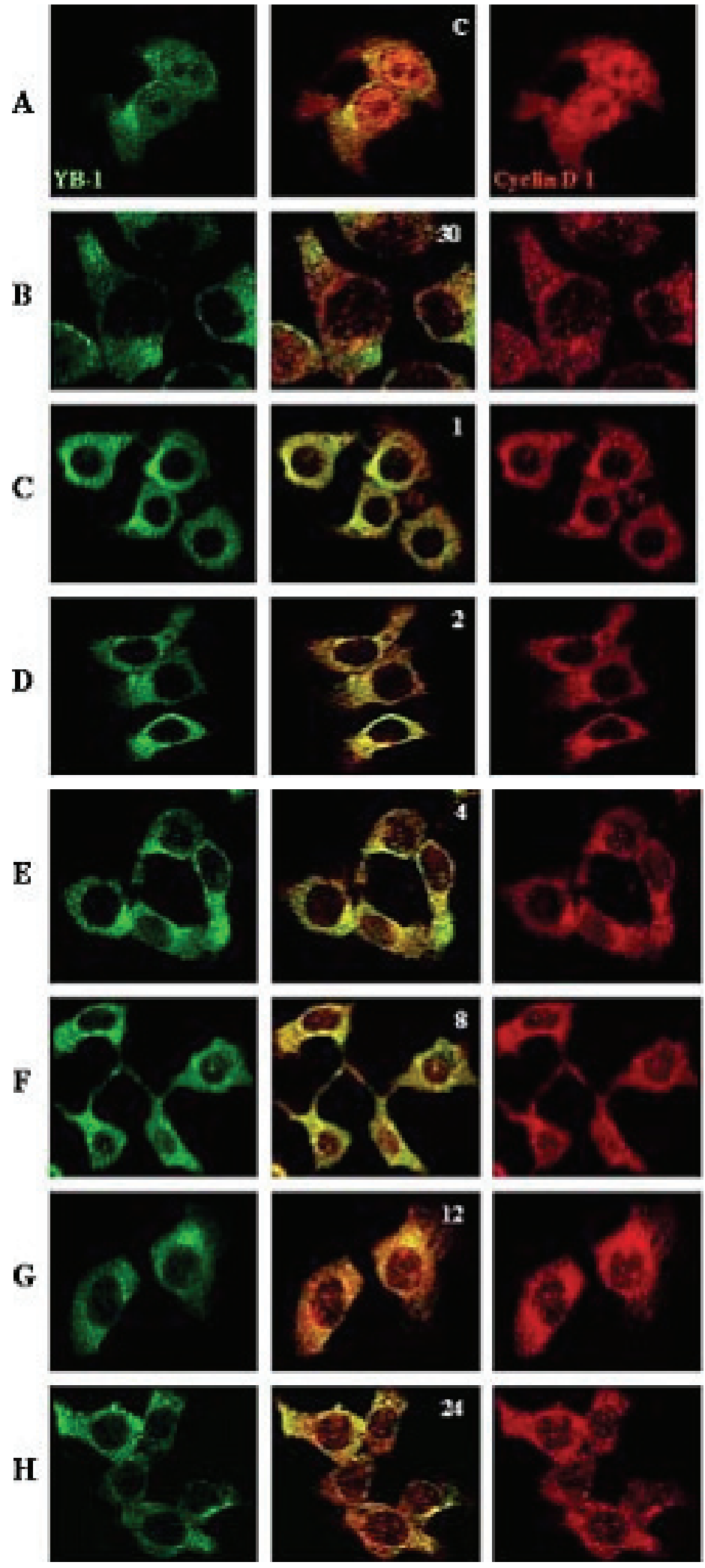
interactions that require for example phosphorylation, or the lack of it can more realistically be assessed.

But there are also some disadvantages of using co-immunoprecipitation assays. These include i) this assay system is not as sensitive as some other techniques like protein affinity chromatography, because the concentration of the antigen is lower than it is in protein affinity chromatography, ii) co-immunoprecipitation of YB-1 with cyclin D1 and vice versa does not necessarily indicate that they interact directly, since they can be a part of some larger complexes in the cell, like in case of E1A and cyclin A proteins (Harlow et al., 1986; Ewen et al., 1992; Faha et al., 1992). In fact, we detected from our preliminary experiments that both YB-1 and cyclin D1 immunoprecipitated with two nuclear factor kappa B (NF-кB) subunits, namely p52 and Rel B. YB-1 was already shown to interact with the NF- $\mathrm{B}$ subunit p65 or Rel A, to modulate its interaction to DNA (Raj et al., 1996). NF- $\mathrm{B}$ was shown to be involved in transcriptional regulation of cyclin D1 (Guttridge et al., 1999). However NF- $\kappa$ B independent activation of cyclin D1 by Rac was shown in MEFs (Klein et al., 2007). It is possible that the YB-1 - cyclin D1 interaction is mediated by the two NF- $\mathrm{BB}$ subunits and they are all part of a bigger protein complex.

Co-localization gives evolutionary processes the opportunity to convert nonspecific binding interactions into interactions that have functional consequences (Kuriyan et al., 2007). Protein-protein interactions help in recruiting cytoplasmic polypeptides to activated receptors, directing their assembly into larger complexes and targeting them to defined subcellular locations. Therefore it was imperative to see if the YB-1 and cyclin D1 proteins colocalize in cells. YB-1 is predominantly localized in the cytoplasm, mainly in the perinuclear space. Cellular stresses like hyperthermia (Stein et al 2001), UV irradiation (Koike et al. 1997) and anti-cancer drugs result in partial translocation of YB-1 protein from cytoplasm to nucleus. The nuclear localization of YB-1 protein was shown to be an important prognostic marker for cancer (Shibahara et al. 2001). Cyclin D1 is present in either nucleus or cytoplasm or both, depending on the phase of cell cycle (Lukas et al., 1994; Diehl et al., 1998). But the mechanisms that regulate this periodic subcellular redistribution of cyclin D1 during cell division cycle are not defined.

We detected co-localization between YB-1 and cyclin D1, especially in the cytoplasm of G2/M phase blocked cells. This suggests the likelihood that interaction between YB-1 and cyclin D1 takes place in vivo. Previous studies showed that there is post-transcriptional induction in the levels of cyclin D1 during transition from the S to G2 phase in cell cycle (Yang et al., 2006) and the nuclear cyclin is translocated to the cytoplasm during this transition (Sumrejkanchanakij et al., 2003), resulting in its predominant cytoplasmic distribution. In order to rule out the possibility of predominant immunoprecipitation of cyclin D1 with YB-1 and vice versa from G2/M phase cells due to the physical presence of both proteins in high concentrations in the same subcellular compartment, we analyzed the nuclear + cytoplasmic extracts of control cells, thereby putting both proteins together in the same fraction. The fact that we do not detect increased immunoprecipitation of YB-1 with cyclin D1 and vice versa in these cells suggests that this interaction is not post lysis. 
The predominant distribution of cyclin D1 in the cytoplasm of G2/M phase blocked cells and its co-localization with YB-1 can be attributed to many reasons. The cytoplasmic cyclin D1 seen in the G2/M cells might be the product of export of the nuclear cyclin D1 seen in the wild type and G1/S phase blocked cells into the cytoplasm. However cyclin D1 has been reported to be a highly unstable protein with a half-life ranging from 13 to $40 \mathrm{~min}$. at the maximum, depending on the cell growth conditions and the phase of cell cycle (Matsushime et al., 1991; Diehl et al., 1998). Besides cyclin D1 is transported to the cytoplasm for proteolysis. So it is highly unlikely that the cytoplasmic cyclin D1 seen is the product of nuclear export. Since cyclin D1 degradation by polyubiquitination occurs in cytoplasm (Diehl et al., 1997; Alt et al., 2000), its absence from the nucleus can be attributed to nuclear export for proteolysis, but the predominant cytoplasmic cyclin D1 is not a result of this nuclear export. There is also a possibility that the cytoplasmic cyclin D1 might be a combination of the newly synthesized and the preexisting cytoplasmic cyclin D1.

The cyclin D1 seen in the cytoplasm may be just the newly synthesized product and YB-1 might play a role in maintaining this newly formed cyclin D1 in the cytoplasm. From the literature, this seems to be the most likely explanation. Cyclin D1 is expressed at low levels during the S-phase to allow sufficient DNA synthesis (Pagano et al., 1994) but then induced to high levels in the G2 phase through Ras activity to commit the cells to continuing the cell cycle progression (Sherr et al., 1992; Aktas et al., 1997; ConnelCrowley et al., 1998; Albercht et al., 1999; Hitomi et al., 1999). Cyclin D1 gets excluded from the nucleus after the S-phase (Baldin et al., 1993; Guo et al., 2005) and this nuclear export has been shown to be mediated by its phosphorylation by glycogen synthase kinase-3 $\beta$ (GSK-3 $\beta$ ), in association with nuclear exportin, CRM1 (Diehl et al., 1998; Alt et al., 2000). Cyclin D1 lacks a nuclear localization signal and under the influence of p21 ${ }^{\text {Cip1 }}$ gets associated with CDK4 to get translocated to the nucleus (Alt et al., 2002) and enter the nucleus only at the G1 phase (Baldin et al., 1993). There is a possibility that YB-1 sequesters this cyclin D1 in the cytoplasm of G2/M phase cells.

In order to investigate if the N-terminus of YB-1 plays a role in its interaction with cyclin D1, we relied on co-immunoprecipitation assays performed on cells incubated with the four APGFP fusion proteins. We show immunoprecipitation of cyclin D1 with fusion proteins APYB26GFP and APYB77GFP from G2/M phase blocked cells. Since cyclin D1 gets immunoprecipitated only in cells incubated with APYB77GFP and APYB26GFP, it is most likely that the AP rich sequence AAPPAAPALSAADTK, missing from the clone APYB36GFP, is atleast atleast to a certain extent responsible for mediating YB-1 binding to cyclin D1 either directly or indirectly. Cyclin D1 is translocated to the cytoplasm during S/G2 transition and failure of re-entry of cyclin D1 into nucleus is known to block cells at the G2 phase of cell cycle. YB-1 is also found in the cytoplasm at the G2 phase. Our current study shows that YB-1 predominantly binds to cyclin D1 in the cytoplasm of G2/M phase cells. Our previous study as described in the second chapter showed that ectopic introduction of APYB77GFP and APYB26GFP into rat hepatoma cells led to $\mathrm{G} 2 / \mathrm{M}$ phase cell cycle arrest and apoptosis. We suggest the possibility that this interaction of cyclin D1 with the N-terminus of YB-1 results in its sequestration in the cytoplasm of G2/M phase blocked cells. Cyclin D1 has been shown 
to be sequestered in the cytoplasm of cardiomyocytes and post mitotic neurons where its activity might be regulated through cytoplasmic sequestration (Tamamori-Adachi et al., 2003; Sumrejkanchanakij et al., 2003). Cyclin D1 interacts with CDK4/6 at all stages and so should be able to translocate to the nucleus. The mechanism behind cytoplasmic sequestration of cyclin D1 is not understood. We speculate that YB-1 binding to cyclin D1 at this stage might result in its sequestration.

Co-immunoprecipitation of cyclin D1 is not seen in unsynchronized cells. This difference can be attributed to spatial constraints resulting from the localization of both proteins in different sub-cellular compartments in unsynchronized cells. Since the three APYBGFP proteins are seen restricted to the cytoplasm and the cyclin D1 is predominantly present in the nucleus of unsynchronized cells, minimal interaction of cyclin D1 with APYBGFP would have been possible.

Posttranslational protein modifications, most notably phosphorylation, represent a common mechanism through which signaling systems are controlled. This regulatory device is inseparably linked with the physical interactions of signaling proteins, since protein phosphorylation commonly exerts its effects by creating binding sites for a set of protein interaction domains, whose ability to bind to their targets is phosphorylation dependent. Phosphorylation can also be viewed as a device to control the assembly of protein complexes and thereby to regulate the dynamic behavior of the cell. In order to understand more about the nucleo-cytoplasmic shuttling of YB-1 and cyclin D1 seen especially in the G2/M phase of cell cycle, we investigated if the phosphorylation states of either of the proteins important for their interaction and/or co-localization with each other.

We report that incubation of nocodazole blocked cells with wortmannin, which is a pharmacological inhibitor of the PI3K-AkT pathway through which YB-1 is phosphorylated, changes the localization pattern of cyclin D1. It has been shown that unphosphorylated YB-1 translationally represses cyclin D1 mRNA (Evdokimova et al., 2006) and once it is phosphorylated, it unbinds from the mRNA and thus cyclin D1 mRNA is free to get translated. According to the previous literature, protein levels of cyclin D1 are maximal in the G2 phase of cells cycle (Guo et al., 2002) and this increase is due to an increase in translational rate, as the transcription rate of cyclin D1 is steady at the S/G2 phase (Guo et al., 2002). This and the fact that cyclin D1 half life is very short led us to believe that it is most likely that the cytoplasmic cyclin D1 seen in nocodazole blocked cells is newly synthesized. Therefore YB-1 is more likely than not in its phosphorylated state. YB-1 is phosphorylated at its Ser102 position by the PI3K-AkT pathway (Sutherland et al., 2005). This phosphorylation of YB-1 is totally inhibited by incubation of cells with wortmannin (Evdokimova et al., 2006).

Simultaneous incubation of cells with nocodazole and wortmannin should block the cells in G2/M phase as well as render the YB-1 unphosphorylated. We confirmed the decrease in phosphorylated YB-1 in wortmannin blocked cells by immunoblotting analysis of whole cell extracts with phospho-serine antibody (data not shown). Under these conditions cyclin D1 was present in both cytoplasm and nucleus, as opposed to its 
predominant cytoplasmic presence in only nocodazole blocked cells. This suggests that YB-1 phosphorylation is probably directly or indirectly important for the sub-cellular localization of cyclin D1 at the G2/M phase of cell cycle. However, the PI3K pathway is involved in regulating many downstream targets other than YB-1 like FKHR, mTOR, p70S6K and Mdm2. Therefore we cannot conclude that YB-1 phosphorylation is indeed directly responsible for the change in cyclin D1 distribution, but it is a strong possibility. We can rule out the GSK-3 $\beta$ pathway involvement in this process since wortmannin blocking of the PI3K phosphorylation pathway renders the GSK-3 $\beta$ active to transport the cyclin D1 into the cytoplasm and yet cyclin D1 is still seen in the nucleus.

A block in cyclin D1 phosphorylation using $\mathrm{LiCl}$ did not change the pattern of distribution of YB-1 or cyclin D1 after nocodazole block, suggesting that this interaction might be independent of the phosphorylation state of cyclin D1. However, it has been shown that cyclin D1 cytoplasmic export is dependent on its phosphorylation by GSK-3 $\beta$ (Alt et al., 2000). But since cyclin D1 proteolysis cannot occur in nucleus, the most likely explanation for its nuclear absence is nuclear export. Some studies have indeed demonstrated that cyclin D1 ubiquitylation and its rapid degradation can occur independently of GSK-3 $\beta$ under certain condition (Germain et al., 2000; Zou et al., 2004). The previous literature and our results combined together suggest that there are many more facets to cyclin D1 nuclear export and YB-1 - cyclin D1 interaction and subcellular translocation is a possible step in this process.

In depth analysis of the importance of phosphorylation in YB-1 - cyclin D1 interaction has to be done to gain a better understanding. Previous studies have shown that phosphorylation dependent protein-protein interactions can easily be studied using yeast or bacterial two-hybrid systems (Guo et al., 2004; Shaywitz et al., 2002). Quantitative proteomics techniques have been employed to obtain information regarding phosphorylation status and signal dependent protein interactions in signal transduction cascades (Patterson et al., 2003).

Since YB-1 and Cyclin D1 bind to the same sub-units of NF- $\kappa$ B complex, p52 and Rel B and cyclin D1 has NF- $\kappa \mathrm{B}$ binding site in its promoter region, there is a possibility that YB-1 might be involved in indirect transcriptional regulation of cyclin D1 by this binding with $\mathrm{p} 52$ and Rel B. Transcriptional block with actinomycin D showed a time dependent change in localization pattern of YB-1 and cyclin D1. The results obtained can be attributed to various possibilities including i) After actinomycin block the cells cannot proceed with cell cycle and therefore get blocked most probably in G2/M phase, since G2/M phase synchronized cells showed the same pattern of distribution of the two proteins as seen till nearly the $4 \mathrm{hrs}$. actinomycin block. ii) Since the cyclin D1 half-life is quite short, most of the nuclear cyclin D1 is degraded by $30 \mathrm{~min}$. to $1 \mathrm{hr}$. time points and the cytoplasmic cyclin D1 seen may be the product of newly translated protein from preexisting mRNAs. iii) Cyclin D1 nuclear import is being prevented until nearly the 4-8hrs time point that might potentially be caused by YB-1.

We propose the possibility that the YB-1 - cyclin D1 interaction is one of the various mechanisms by which YB-1 is involved in mediation of cell proliferation. The 
final conclusion from this part of the project is that YB-1 interacts with cyclin D1 at the G2/M phase and probably sequesters it in the cytoplasm, thereby regulating the cell proliferation. We believe that targeting this interaction may help us in understanding the role played by the upregulation of these proteins in the aberrant cells. It might eventually help us to develop mechanisms to prevent their upregulation and thereby decrease the proportion of cells undergoing tumorigenesis. Characterizing this specific complex seen at all phases of cell cycle may offer novel strategies for the development of new anticancer drugs. 


\section{Chapter 4. Summary and Overview}

\subsection{Summary}

YB-1 expression is closely associated with cell proliferation; for example, YB-1 is highly expressed in regenerating liver and liver cancers but barely detectable in normal adult liver (Grant et al., 1993; Kandala et al., 1994). The domain/domains of YB-1 potentially involved in regulation of cell proliferation and the molecular mechanism behind this regulation has not been described. Our previous study demonstrated that disruption of one allele of Chk-YB-1b gene in DT-40 cells at its N-terminal domain resulted in growth defects in the heterozygous mutants (Swamynathan et al., 2002). We hypothesized that the abnormalities seen were due to a dominant negative effect exerted by the putative truncated protein product of the disrupted allele.

The first goal of this project was to determine if the YB-1 N-terminus is involved in regulation of cell proliferation by investigating whether the cell cycle defects in heterozygous mutant DT-40 cells could be recapitulated by introducing YB-1 N-terminal purified proteins into mammalian cell lines. We demonstrated that introduction of the fusion proteins reproduced the G2/M phase block and apoptosis seen in the DT-40 mutants. These results confirm our hypothesis that the N-terminal domain of YB-1 is involved in regulation of cell proliferation. The second part of the project was to investigate the molecular mechanism behind this regulation of cell proliferation by YB-1. Our study for the first time showed a protein-protein interaction between YB-1 and cyclin D1 in the cytoplasm of cells in G2/M phase of cell cycle and we propose that this interaction might be one of the molecular mechanisms behind the regulation of cell proliferation by YB-1. These results are summarized in Fig 4.1.

\subsection{Discussion}

\subsubsection{Potential YB-1 N-terminal sequences playing a role in cell proliferation}

We tested the influence of three YB-1 N-terminal sequences on cell proliferation. APYB77GFP incubation resulted in maximum $\mathrm{G} 2 / \mathrm{M}$ phase cell cycle arrest and apoptosis. APYB36GFP incubation showed the least effect. This variation could be due to multiple reasons. One of the possibilities is that the proline alanine rich sequence in APYB77GFP and APYB26GFP, which is absent from APYB36GFP, might be solely responsible for this difference by its direct binding to some cell cycle protein. Alternatively, this proline alanine rich sequence might aid in proper folding/unfolding and configuration of the fusion proteins and the lack of it might result in a different three dimensional structure of APYB36GFP, thereby rendering it less functional by either hiding the sequence responsible for causing the defects or by some other mechanism. Another possible explanation is that the sequences flanking the proline rich sequence might also have a role to play in mediating these defects. Since APYB36GFP fusion 


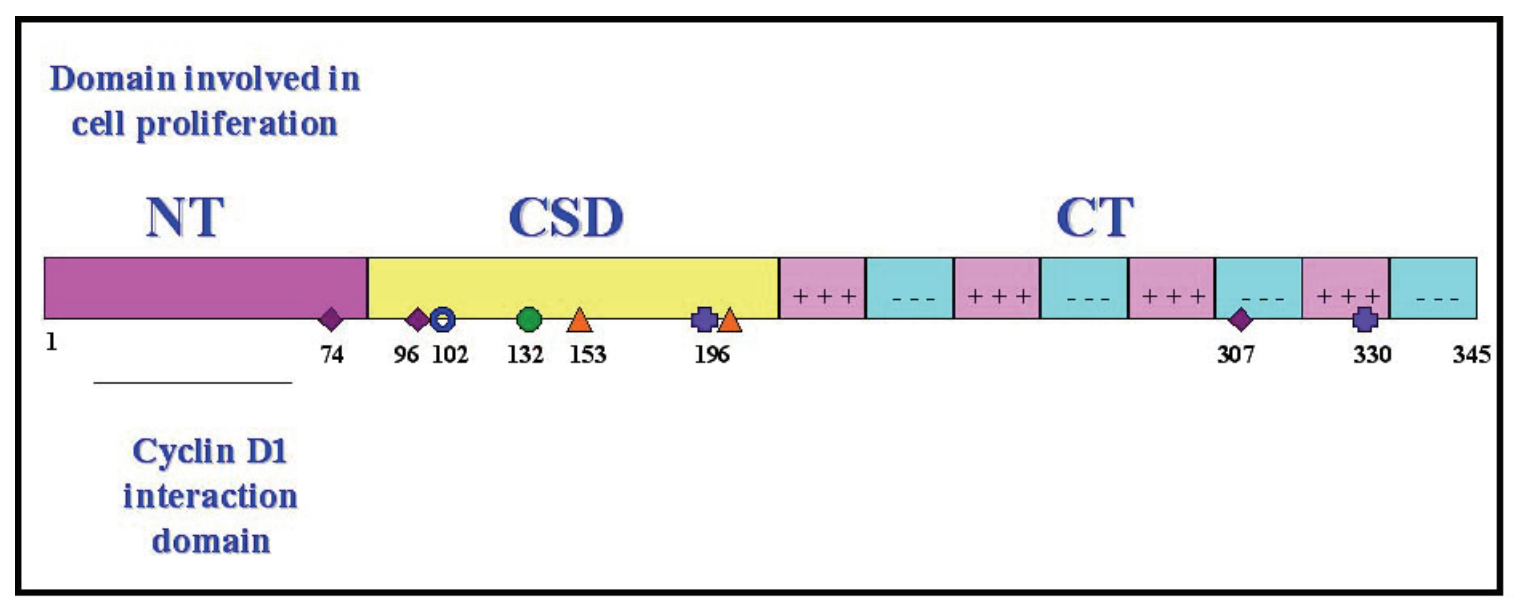

Fig 4.1 Summary of the results of this study

We showed that the N-terminal domain of YB-1 plays a role in cell proliferation and apoptosis. The YB-1 N-terminus is also involved in binding to the cell cycle protein cyclin D1 in the cytoplasm of cells in G2/M phase of cell cycle. 
protein has some of the flanking sequences, it might show minimal defects in the cell growth. There is also a possibility that the proline rich sequence might be functioning just as a spacer between its flanking sequences in order for them to exert their effects and the lack of this spacer in the APYB36GFP protein brings together two stretches of sequences which might render lesser competency to aid in regulating cell proliferation. The APYB26GFP incubation showing lesser effect on cell cycle than the APYB77GFP suggests that the APYB26GFP lacks some amino acids required for maximal effect. The APYB36GFP incubation resulting in significantly lesser effect, suggests that it has some amino acids required for exerting this effect but lacks most of them. Therefore the most likely explanation for this differential response would be that the proline rich sequence along with some other amino acids, most likely the ones flanking this sequence, might be mediating this YB-1 regulation of cell proliferation.

\subsubsection{YB-1 N-terminus interacts with cyclin D1 in the cytoplasm of G2/M phase cells}

We show interaction of YB-1 with cyclin D1 in the cytoplasm of G2/M phase blocked cells. Further analysis with YB-1 N-terminal fusion proteins revealed that cyclin D1 immunoprecipitated only with APYB77GFP or APYB26GFP from G2/M phase blocked whole cell extracts, suggesting not only that the YB-1 N-terminus is involved in the interaction, but also that the short proline alanine rich sequence -

AAPPAAPALSAADTK is crucial for this interaction. Since incubation with the same two fusion proteins resulted in maximal cell cycle arrest and apoptosis, we suggest the possibility that this proline alanine rich sequence binds and sequesters the cyclin D1 in the cytoplasm of $\mathrm{G} 2 / \mathrm{M}$ phase cells thereby rendering a block in the progression of cell cycle. But lower proportion of $\mathrm{G} 2 / \mathrm{M}$ phase arrested and apoptotic cells observed with APYB26GFP compared to APYB77GFP suggests that the proposed cyclin D1 interaction with the proline rich sequence is not solely involved in mediating this arrest. The other amino acid residues present in the YB77 fusion protein but absent from the YB26 protein might also have a role to play in this process.

\subsection{Hypothesis}

We propose that the binding of YB-1 N-terminal proline rich sequence with cyclin D1 in the cytoplasm of G2/M phase cells, sequesters the cyclin D1 and restricts its reentry into the nucleus for cell cycle progression, resulting in an arrest at the G2/M phase and apoptosis. We put forth a model (Fig. 4.2) to explain our hypothesis. In normal cells, YB-1 regulates cyclin D1 tranlation by binding to its mRNA. External stimuli (step 1) causing phosphorylation of YB-1 releases it from the cyclin D1 mRNA (step 2) which is now free to be translated (Evdokimova et al., 2006). Once the cyclin D1 protein is formed (step 3), it binds with CDK4/6 (step 4) for its nuclear entry (step 5). Nuclear cyclin D1 helps in cell cycle progression from the G1 to S phase (step 6), after which the cyclin D1 is excluded from the nucleus (step 8). Therefore during cell cycle transition from S to G2 phase, cyclin D1 is found in the cell cycoplasm. This cyclin D1 may be a product of the nuclear export (step 8) and/or new translation (step 9). Simulation of the G2/M phase in 


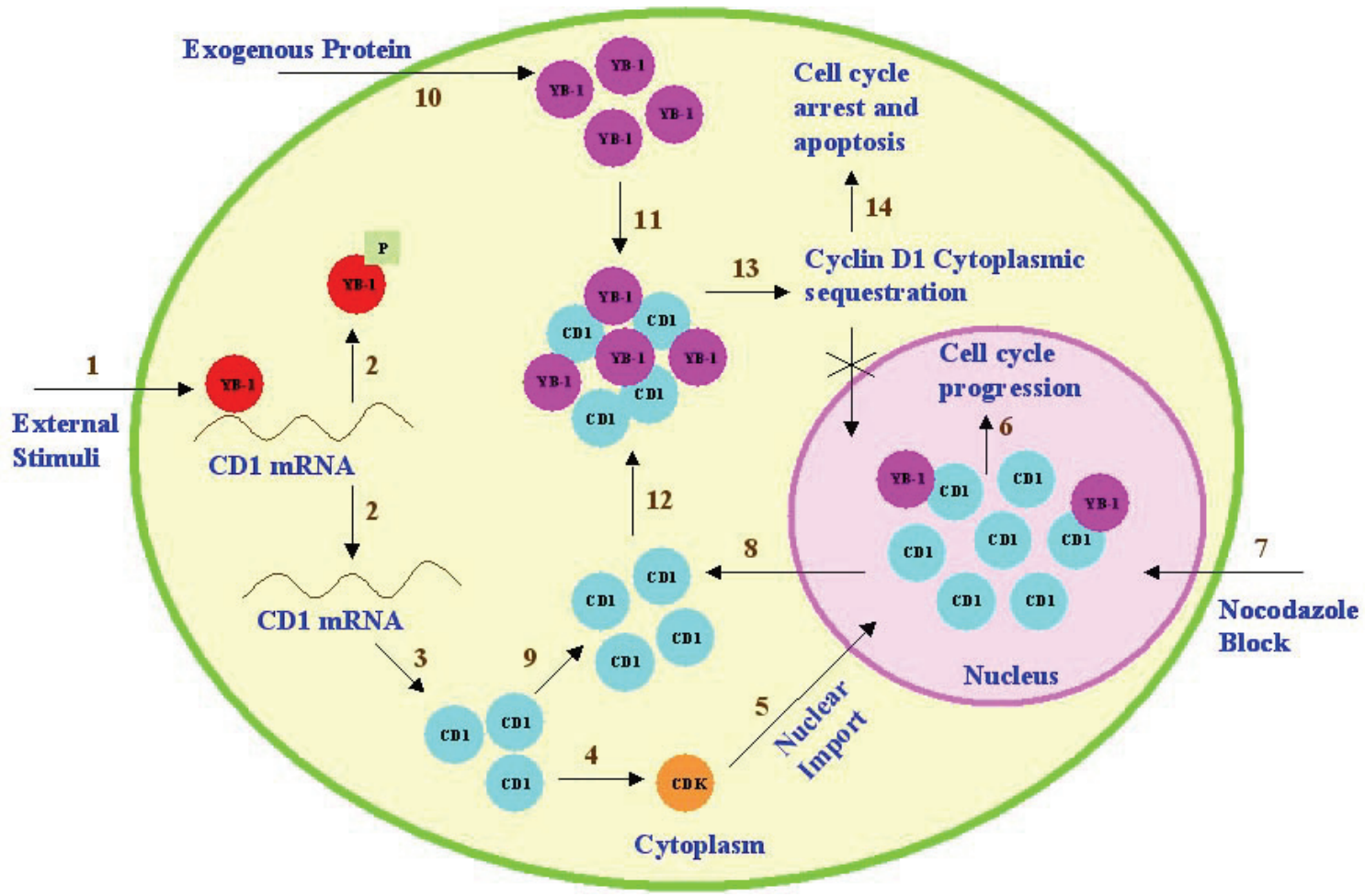

Fig 4.2 Model of cyclin D1 sequestration by binding to YB-1 N-terminal proteins in the cytoplasm of $\mathrm{G} 2 / \mathrm{M}$ phase cells leading to cell cycle arrest

The model can be explained in different steps represented by numbers for simplification. Unphosphorylated YB-1 binds and represses cyclin D1 mRNA translation. External stimuli (1) activating the PI3K-AkT pathway causes YB-1 phosphorylation resulting in release of the cyclin D1 mRNA (2). The new cyclin D1 protein interacts with CDK4/6 (3 and 4), translocates to the nucleus (6) and aids in G1 to S phase cell cycle progression (6). Further progression of cell cycle to the G2/M phase requires cytoplasmic translocation of cyclin D1(8). We simulated the G2/M phase in cells by a nocodazole block (7). We propose that under conditions when the cell is targeted for apoptosis, the YB-1 N-terminal proteins (10) bind to the cyclin D1 (11 and 12) and sequester them in the cytoplasm (13), resulting in cell cycle arrest at the G2/M phase and ultimately apoptosis (14). 
our cells with a nocodazole block (step 7), along with introduction of the YB-1 Nterminal proteins (step 10), resulted in cell cycle arrest and apoptosis (Figs. 2.13 to 2.16). We propose that the YB-1 N-terminus binds with this cyclin D1 (steps 11 and 12) and restricts its entry/re-entry into the nucleus (step 13), resulting in an arrest in cell cycle at the G2/M phase and apoptosis (step 14). The broad idea behind this hypothesis is that once the cell fate is determined, different pathways are activated (step 1) to prepare the cell for either another round of division (steps 5 and 6) or cell cycle arrest (steps 8 to 14). So when the cell has to continue cell division, N-terminus of YB-1 does not interact with cyclin D1 (steps 11 and 12), which can then translocate to the nucleus for another round of division (steps 4-6). But when the cells have to stop dividing, the YB-1 N-terminus binds to cyclin D1 (step 11 and 12), sequestering it in the cytoplasm (step 13) and arresting the cells at the G2/M phase of cell cycle (step 14), by hitherto unknown mechanisms.

We also suspect that YB-1 regulates the cyclin D1 at both translational and posttranslational levels. Unphosphorylated YB-1 binds to cyclin D1 mRNA (Evdokimova et al., 2006) in the cytoplasm and represses its translation. Once YB-1 is phosphorylated (step 1), cyclin D1 translational block is released, new cyclin D1 protein is synthesized, which binds with CDK4/6 to enter the nucleus and aid in cell cycle progression from G1 to S phase (steps 3 to 6), after which the cyclin D1 translocates to the cell cytoplasm (step 8). We saw from our experiments that under normal conditions, YB-1 and Cyclin D1 interaction was seen to be predominant in the cytoplasm of G2/M phase cells (Figs 3.4 to 3.6). But under a wortmannin block where YB-1 phosphorylation is inhibited, cyclin D1 is found in the nucleus at the G2/M phase (Fig. 3.8), suggesting the possibility of phosphorylated YB-1 binding to the nuclear cyclin D1 to aid its cytoplasmic transport at the G2/M phase. YB-1 is phosphorylated at the Ser102 position in its CSD domain by the PI3K-AkT pathway (Sutherland et al., 2005), which is inhibited in this case by wortmannin incubation (Evdokimova et al., 2006). So there is a possibility that through different domains in its protein, YB-1 regulates the cell proliferation in different ways. When the cell proliferation has to be controlled, its N-terminus interacts with the cytoplasmic Cyclin D1 (step 11 and 12), restricting its nuclear entry, resulting in cell cycle arrest. When cell proliferation has to continue, the $\mathrm{N}$-terminus does not interact with cyclin D1, but the CSD interacts with it in the nucleus for translocation into the cytoplasm thereby helping in cell cycle progression, because a block in cytoplasmic translocation of cyclin D1 results in cell cycle arrest at the G1/S phase. Thus YB-1 might be post-translationally controlling the cyclin D1.

The total levels of YB-1 protein may also be playing a role in this differential regulation of cell proliferation. High amounts of nuclear translocation of YB-1 have been reported in actively proliferating and cancer cells (Jurchott et al., 2003; Oda et al., 2003; Fujita et al., 2005). There is a possibility that this increase in nuclear YB-1 aids in the cytoplasmic translocation of cyclin D1 at the S/G2 phase through its CSD, which helps in progression of the cell cycle. In the absence of a stress or proliferative stimuli, most of the cellular YB-1 is cytoplasmic, where its N-terminus can interact with Cyclin D1 in order to control the levels of cell proliferation. We propose that the molecular mechanism behind regulation of cell proliferation by YB-1 involves this complex regulation of 
translational and post translational levels of cyclin D1 and its nucleocytoplasmic transport through binding with YB-1 mediated by its N-terminus and cold shock domain.

\subsection{Future experiments to test our hypothesis}

Testing our hypothesis about cytoplasmic sequestration of cyclin D1 by YB-1 in the $\mathrm{G} 2 / \mathrm{M}$ phase during a nocodazole block is relatively easy, because of its reversibility by incubating the blocked cells in a nocodazole free medium for about $24 \mathrm{hrs}$. So if the YB-1 N-terminal sequence does sequester the cyclin D1 in cell cytoplasm resulting in an arrest at the G2/M phase, removal of the nocodazole from the incubation medium should not decrease the cell cycle arrest and apoptosis of the cells. The cyclin D1 should still get immunoprecipitated with APYB77GFP and APYB26GFP.

A way of testing if interaction of the N-terminal YB-1 proteins with cyclin D1 results in cell cycle arrest is to supplement the cells with increasing concentrations of proteins competing for binding with either YB-1 (example p53) or cyclin D1 (example CDK4/6). If the dose dependent increase in competing proteins immunoprecipitating YB-1 or cyclin D1 results in an decrease of immunoprecipitation of cyclin D1 with either of the N-terminal YB-1 proteins, resulting in comparable decrease in the cell cycle arrest and apoptosis, it would suggest that the defects seen are indeed due to the interaction between YB-1 N-terminal proteins with cyclin D1. Our results and this hypothesis is in agreement with some previously published studies, showing that Cyclin D1 cytoplasmic sequestration results in cell cycle arrest at the G2/M phase (Alao et al., 2006). The mechanism behind cytoplasmic sequestration of cyclin D1 has not been understood and our data provides a new avenue for further exploration of the possibility of the Nterminus of YB-1 causing this sequestration.

But even if the YB-1 N-terminal proteins do sequester cyclin D1 in the cell cytoplasm resulting in the cell cycle arrest, the proline rich sequence might not necessarily be involved in this interaction. This sequence might only be important for protein folding or separating the two flanking sequences. The importance of these sequences can be checked by constructing fusion proteins with i) only the proline rich sequence, ii) only the sequences flanking this proline rich sequence and iii) the flanking sequences along with the a random spacer sequence instead of the proline rich sequence. Structural analysis of the APYBGFP fusion proteins might also give us a fair idea of the importance of this proline rich sequence in protein folding and the residues which are exposed in each of the fusion proteins for taking part in protein-protein interactions.

We have a custom peptide constituting of only the 26 proline-alanine rich amino acids present in APYB26GFP. This peptide can be exploited for i) investigating if this sequence is enough for interaction with cyclin D1. Peptide bound beads can be used for affinity chromatography of whole cell extracts followed by proteomic analysis, in order to investigate if cyclin D1 gets immunoprecipitated with this sequence. This procedure will also enable identification of any other proteins, which bind with the proline alanine rich sequence. Differential bindings can also be analyzed by using different sub-cellular 
extracts or extracts prepared after various treatments. ii) After the binding assays, this peptide can also be used to investigate if cytoplasmic cyclin D1 sequestration is seen by its introduction into cells. If the YB-1 proline rich sequence interacts with and sequesters cyclin D1 in the cell cytoplasm resulting in cell cycle arrest, introduction of this peptide should result in ultimate apoptosis. iii) This peptide can also to be used as a competing protein for binding of the cytoplasmic cyclin D1 with APYB77GFP or APYB26GFP. If indeed this sequence is sufficient for binding with cyclin D1, the competitive assay should reduce the levels of cyclin D1 pull down with the GFP antibody from APYB77GFP or APYB26GFP incubated cells, while still maintaining similar levels of cell cycle arrest and apoptosis. iv) We can also explore the possibility of complimenting the peptide positive cells with a fusion protein missing this exact sequence, in order to test the importance of the 77 amino acid YB-1 N-terminal sequence in inducing cell cycle arrest and apoptosis.

From our localization analysis of YB-1 and cyclin D1 under wortmannin and LiCl incubations, we had proposed the possibility of phosphorylated YB-1 being important for the predominant cytoplasmic cyclin D1 localization in $\mathrm{G} 2 / \mathrm{M}$ phase. This possibility can be tested by using an YB-1 CSD Ser102 mutant for similar localization analysis. YB-1 is phosphorylated by the PI3K-AkT pathway at this position and this phosphorylation is completely inhibited by wortmannin. If YB-1 phosphorylation is important for cyclin D1 cytoplasmic localization at the $\mathrm{G} 2 / \mathrm{M}$ phase, use of this mutant protein should not result in predominant cytoplasmic cyclin D1 at the G2/M phase. Since this experiment does not involve the use of wortmannin, we can rule out the possibility of other pathways (which are downstream to the PI3K-AkT) being involved in this process. The caveat of this experiment would be the presence of endogenous wild type YB-1. This can be overcome by initial downregulation of the wild type YB-1 protein by using siRNA specific for either the $\mathrm{N}$-terminus or the $\mathrm{C}$-terminus of YB-1 so that it does not affect our mutant protein. This experiment will extend our understanding of the interaction of YB-1 with cyclin D1.

If we can confirm that the phosphorylation state of YB-1 is important for cyclin D1 cytoplasmic localization in $\mathrm{G} 2 / \mathrm{M}$ phase cells from this experiment, we can look into the future possibility of testing the hypothesis of the YB-1 CSD domain interacting with cyclin D1 for its nuclear export and the YB-1 N-terminal domain interacting with it for cytoplasmic sequestration. The experimental design to test this hypothesis can involve the use of YB-1 deletion mutants expressing i) wild type CSD domain, ii) wild type amino and CSD domains, iii) CSD Ser102 mutant, iv) wild type amino domain and the CSD Ser102 mutant, v) full length YB-1 with just the Ser102 mutant along with the wild type full length protein.

\subsection{Significance - YB-1 and cancer}

YB-1 protein levels are highly upregulated in actively proliferating cells including cancer cells. Numerous studies have also shown that nuclear accumulation of YB-1 is a good predictor of cancer (Bargou et al., 1997; Oda et al., 1998 and 2003; Kamura et al., 
1999; Shibahara et al., 2001; Yahata et al., 2002; Saji et al., 2003; Gessner et al., 2004; Yasen et al., 2005; Fujita et al., 2005; Basaki et al., 2007). Therefore it seems likely that there is significant nuclear accumulation of YB-1 during uncontrolled cell proliferation and predominant cytoplasmic localization in other conditions including apoptotic cells. But we do not know as yet if this increase in proteins in cancer cells is due to increased YB-1 translation or increase in amounts of YB-1 mRNA. Many studies have targeted YB-1 in order to find novel approaches to cancer therapy, albeit with little success. Most recently, some preclinical studies targeted YB-1 in order to develop therapeutic strategies against breast cancer (Fujii et al., 2008). Many prior studies have also shown or indicated importance of similar targeting of YB-1 for various cancer treatments with limited success (Kamura et al., 1999; Janz et al., 2002; Holm et al, 2004; Huang et al., 2005; Mantwill et al., 2006; Vaiman et al., 2007; Stratford et al., 2007). In absence of the knowledge of the exact molecular mechanism, it is difficult to design therapeutic agents against YB-1 for cancer treatment.

The first half of this project was designed to investigate the involvement of the $\mathrm{N}$-terminal domain in an attempt to make targeting of YB-1 for cancer therapy more specific. The second half of this project was designed to broaden the scope of targeting YB-1 for cancer therapy by investigating the association of YB-1 with the cell cycle protein cyclin D1 at specific stages in cell cycle. We found that the N-terminus of YB-1 plays a role in cell proliferation. With this information, further experiments can be designed to investigate if increase in levels of the YB-1 N-terminal proteins in other cancer cells results in a decrease in cell proliferation or increase in cell cycle arrest thereby ultimately aiding in cancer regression. Our study also suggests that the small proline alanine rich sequence (PPAAPPAAPALSAADTK) from the YB-1 N-terminus might be important for the regulation of cell proliferation by YB-1. We also show that this sequence is a strong potential candidate for interaction of YB-1 with cyclin D1 in the cytoplasm of cells in G2/M phase of cell cycle, which might result in the sequestration of cyclin D1 resulting in cell cycle arrest. Analysis of the important of this sequence could pave a definite road into understanding the minimal YB-1 N-terminal sequence required for its involvement in cell proliferation. If further exprements can prove that the cell cycle arrest is indeed due to cytoplasmic sequestration of cyclin D1, this information can be further exploited for use in cancer therapy in association with YB-1 or even independent of YB-1. Therefore the data from this project extends our understanding of

the role of YB-1 in cell proliferation in terms of the specific YB-1 domain mediating this process and a very strong potential mechanism by which it is mediated.

\subsection{Future directions}

Based on the results of this project, future studies can focus on i) importance of YB-1 N-terminal protein binding to cyclin D1 in the cytoplasm of G2/M phase cells in terms of its cytoplasmic sequestration, ii) importance of the non proline rich YB-1 $\mathrm{N}$-terminal sequence in cell proliferation, iii) investigating if the YB-1 CSD is also involved in interaction with cyclin D1 and iv) investigation of the importance of YB-1 Ser102 phosphorylation for its interaction with cyclin D1 and the nucleocytoplasmic 
shuttling of cyclin D1. It might also be interesting to investigate if YB-1 and Cyclin D1 are a part of a larger protein complex consisting of NF- $\mathrm{kB}$ subunits Rel B and $\mathrm{p} 52$ and if YB-1 is involved in the indirect transcription regulation of cyclin D1 gene in association with NF-кB. Ultimately, investigating the importance of YB-1 in cell proliferation will benefit our understanding of how and why it is upregulated in cancer cells. 


\section{List of References}

Agami, R., Bernards, R.. (2000) Distinct initiation and maintenance mechanisms cooperate to induce G1 cell cycle arrest in response to DNA damage. Cell. 102, 55-66.

Aktas, H., Cai, H., Cooper, G.M. (1997) Ras links growth factor signaling to the cell cycle machinery via regulation of cyclin D1 and the Cdk inhibitor p27KIP1. Mol Cell Biol. 17, 3850-3857.

Alao, J.P., Gamble, S.C., Stavropoulou, A.V., Pomeranz, K.M., Lam, E.W., Coombes, R.C., Vigushin, D.M. (2006) The cyclin D1 proto-oncogene is sequestered in the cytoplasm of mammalian cancer cell lines. Mol Cancer. 5, 7.

Albanese, C., Johnson, J., Watanabe, G., Eklund, N., Vu, D., Arnold, A., Pestell, R.G. (1995) Transforming $\mathrm{p} 21$ ras mutants and c-Ets-2 activate the cyclin D1 promoter through distinguishable regions. J Biol Chem. 270, 23589-23597.

Albrecht, J.H., Hansen, L.K. (1999) Cyclin D1 promotes mitogen-independent cell cycle progression in hepatocytes. Cell Growth Differ. 10, 397-404.

Alt, J.R., Cleveland, J.L., Hannink, M., Diehl, J.A. (2000) Phosphorylation-dependent regulation of cyclin D1 nuclear export and cyclin D1-dependent cellular transformation. Genes Dev. 14, 3102-3114.

Alt, J.R., Gladden, A.B., Diehl, J.A. (2002) p21 (Cip1) Promotes cyclin D1 nuclear accumulation via direct inhibition of nuclear export. J Biol Chem. 277, 8517-8523. Arnold, A. (1995) The cyclin D1/PRAD1 oncogene in human neoplasia. J Investig Med. $43,543-549$.

Asakuno, K., Kohno, K., Uchiumi, T., Kubo, T., Sato, S., Isono, M., Kuwano, M. (1994) Involvement of a DNA binding protein, MDR-NF1/YB-1, in human MDR1 gene expression by actinomycin D. Biochem Biophys Res Commun. 199, 1428-1435.

Ashizuka, M., Fukuda, T., Nakamura, T., Shirasuna, K., Iwai, K., Izumi, H., Kohno, K., Kuwano, M., and Uchiumi, T. (2002) Novel translational control through an ironresponsive element by interaction of multifunctional protein YB-1 and IRP2. Mol Cell Biol. 22, 6375-6383.

Bader, A.G., Vogt, P.K. (2005) Inhibition of protein synthesis by Y box-binding protein 1 blocks oncogenic cell transformation. Mol Cell Biol. 25, 2095-2106.

Balda, M.S., Garrett, M.D., Matter, K. (2003) The ZO-1-associated Y-box factor ZONAB regulates epithelial cell proliferation and cell density. J Cell Biol. 160, 423-432. 
Balda, M.S., Matter, K. (2000) The tight junction protein ZO-1 and an interacting transcription factor regulate ErbB-2 expression. EMBO J. 19, 2024-2033.

Baldin, V., Lukas, J., Marcote, M.J., Pagano, M., Draetta, G. (1993) Cyclin D1 is a nuclear protein required for cell cycle progression in G1. Genes Dev. 7, 812-821.

Bargou, R.C., Jurchott, K., Wagener, C., Bergmann, S., Metzner, S., Bommert, K., Mapara, M.Y., Winzer, K.J., Dietel, M., Dorken, B., and Royer, H.D. (1997) Nuclear localization and increased levels of transcription factor YB-1 in primary human breast cancers are associated with intrinsic MDR1 gene expression. Nat Med. 3, 447-450.

Basaki, Y., Hosoi, F., Oda, Y., Fotovati, A., Maruyama, Y., Oie, S., Ono, M., Izumi, H., Kohno, K., Sakai, K., Shimoyama, T., Nishio, K., Kuwano, M. (2007) Akt-dependent nuclear localization of Y-box-binding protein 1 in acquisition of malignant characteristics by human ovarian cancer cells. Oncogene. 26, 2736-2746.

Bergmann, S., Royer-Pokora, B., Fietze, E., Jürchott, K., Hildebrandt, B., Trost, D., Leenders, F., Claude, J.C., Theuring, F., Bargou, R., Dietel, M., Royer, H.D. (2005) YB1 provokes breast cancer through the induction of chromosomal instability that emerges from mitotic failure and centrosome amplification. Cancer Res. 65, 4078-4087.

Bezzubova, O., Silbergleit, A., Yamaguchi-Iwai., Y, Takeda, S., and Buerstedde, J.M. (1997) Reduced X-ray resistance and homologous recombination frequencies in a RAD54-/- mutant of the chicken DT40 cell line. Cell. 89, 185-193.

Binder, H., and Lindblom, G. (2003) Charge-dependent translocation of the Trojan peptide penetratin across lipid membranes. Biophys J. 85, 982-995.

Bouvet, P., Matsumoto, K., Wolffe, A.P. (1995) Sequence-specific RNA recognition by the Xenopus Y-box proteins. An essential role for the cold shock domain. J Biol Chem. 270, 28297-28303.

Brown, J.R., Nigh, E., Lee, R.J., Ye, H., Thompson, M.A, Saudou, F., Pestell, R.G., Greenberg,M.E. (1998) Fos family members induce cell cycle entry by activating cyclin D1. Mol Cell Biol. 18, 5609-5619.

Buerstedde, J.M., and Takeda, S. (1991) Increased ratio of targeted to random integration after transfection of chicken B cell lines. Cell. 67, 179-188.

Casanovas, O., Miró, F., Estanyol, J.M., Itarte, E., Agell, N., Bachs, O. (2000) Osmotic stress regulates the stability of cyclin D1 in a p38SAPK2-dependent manner. J Biol Chem. 275, 35091-35097.

Chansky, H.A., Hu, M., Hickstein, D.D, and Yang, L. (2001) Oncogenic TLS/ERG and EWS/Fli-1 fusion proteins inhibit RNA splicing mediated by YB-1 protein. Cancer Res. 61, 3586-3590. 
Chatterjee, M., Rancso, C., Stuhmer, T., Eckstein, N., Andrulis, M., Gerecke, C., Lorentz, H., Royer, H.D., Bargou, R.C. (2007) The Y-box binding protein YB-1 is associated with progressive disease and mediates survival and drug resistance in multiple myeloma. Blood. Nov 15 [Epub ahead of print]

Chen, N.N., and Khalili, K.. (1995) Transcriptional regulation of human JC polyomavirus promoters by cellular proteins YB-1 and Pur alpha in glial cells. J Virol. 69, 5843-5848.

Cheng, M., Olivier, P., Diehl, J.A., Fero, M., Roussel, M.F., Roberts, J.M., Sherr, C.J. (1999) The p21(Cip1) and p27(Kip1) CDK 'inhibitors' are essential activators of cyclin D-dependent kinases in murine fibroblasts. EMBO J. 18, 1571-1583.

Chernukhin, I.V., Shamsuddin, S., Robinson. A.F., Carne, A.F., Paul, A., El-Kady, A.I., Lobanenkov, V.V., Klenova, E.M. (2000) Physical and functional interaction between two pluripotent proteins, the Y-box DNA/RNA-binding factor, YB-1, and the multivalent zinc finger factor, CTCF. J Biol Chem. 275, 29915-29921.

Choudhuri, S., Klaassen, C.D. (2006) Structure, function, expression, genomic organization, and single nucleotide polymorphisms of human ABCB1 (MDR1), ABCC (MRP), and ABCG2 (BCRP) efflux transporters. Int J Toxicol. 25, 231-259.

Coqueret, O. (2002) Linking cyclins to transcriptional control. Gene. 299, 35-55.

Connell-Crowley, L., Elledge, S.J., Harper, J.W. (1998) G1 cyclin-dependent kinases are sufficient to initiate DNA synthesis in quiescent human fibroblasts. Curr Biol. 8, 65-68.

Crawford, P.A., Sharma, N., Chandrasekar, S., Sigdel, T., Walsh, T.R., Spencer, J., and Crowder, M.W. (2004) Over-expression, purification, and characterization of metallobeta-lactamase ImiS from Aeromonas veronii bv. sobria. Protein Expr Purif. 36, 272-279.

Derossi, D., Calvet, S., Trembleau, A., Brunissen, A., Chassaing, G., and Prochiantz, A. (1996) Cell internalization of the third helix of the Antennapedia homeodomain is receptor-independent. J Biol Chem. 271, 18188-18193.

Dhalla, A.K., Ririe, S.S., Swamynathan, S.K., Weber, K.T., and Guntaka, R.V. (1998) chk-YB-1b, a Y-box binding protein activates transcription from rat alphal (I) procollagen gene promoter. Biochem J. 336, 373-379.

Diamond, P., Shannon, M.F., Vadas, M.A., and Coles, L.S. (2001) Cold shock domain factors activate the granulocyte-macrophage colony-stimulating factor promoter in stimulated Jurkat T cells. J Biol Chem. 276, 7943-7951.

Didier, D.K., Schiffenbauer, J., Woulfe, S.L., Zacheis, M., and Schwartz, B.D. (1988) Characterization of the cDNA encoding a protein binding to the major histocompatibility complex class II Y box. Proc Natl Acad Sci U S A. 85, 7322-7326. 
Diehl, J.A. (2002) Cycling to cancer with cyclin D1. Cancer Biol Ther. 1, 226-231.

Diehl, J.A., Cheng, M., Roussel, M.F., and Sherr, C.J. (1998) Glycogen synthase kinase3beta regulates cyclin D1 proteolysis and subcellular localization. Genes Dev. 12, 34993511.

Diehl, J.A., Zindy, F., Sherr, C.J. (1997) Inhibition of cyclin D1 phosphorylation on threonine-286 prevents its rapid degradation via the ubiquitin-proteasome pathway. Genes Dev. 11, 957-972.

Dorn, A., Bollekens, J., Staub, A., Benoist, C., and Mathis, D. (1987) A multiplicity of CCAAT box-binding proteins. Cell. 50, 863-872.

Du, H.Y., Olivo, M., Chen, Y.J., Yip, G., Tan, P.H., Matsumoto, K., Tsujimoto, M., Bay, B.H. (2005) Expression of Y-box binding protein-1 following hypericin-mediated photodynamic therapy in well-differentiated nasopharyngeal cancer in vivo. Int J Mol Med. 16, 865-868.

Dufourny, B., van, Teeffelen, H.A. , Hamelers, I.H., Sussenbach, J.S., Steenbergh, P.H. (2000) Stabilization of cyclin D1 mRNA via the phosphatidylinositol 3-kinase pathway in MCF-7 human breast cancer cells. J Endocrinol. 166, 329-338.

Duh, J.L., Zhu, H., Shertzer, H.G., Nebert, D.W., and Puga, A. (1995) The Y-box motif mediates redox-dependent transcriptional activation in mouse cells. J Biol Chem. 270, 30499-30507.

Duquesne, F., Florent, M., Roué, G., Troussard X, Sola B. (2001) Ectopic expression of cyclin D1 impairs the proliferation and enhances the apoptosis of a murine lymphoid cell line. Cell Death Differ. 8, 51-62.

En-Nia, A., Yilmaz, E., Klinge, U., Lovett, D.H., Stefanidis, I., and Mertens, P.R. (2005) Transcription factor YB-1 mediates DNA polymerase alpha gene expression. J Biol Chem. 280, 7702-7711.

Eric, G. Moss, Rosalind, C. Lee and Victor, Ambros. (1997) The cold shock domain protein lin-28 controls developmental timing in C. elegans and is regulated by the lin-4 RNA. Cell. 88, 637-646.

Evdokimova, V.M., Ovchinnikov, L.P. (1999) Translational regulation by Y-box transcription factor: involvement of the major mRNA-associated protein, $\mathrm{p} 50$. Int $\mathrm{J}$ Biochem Cell Biol. 31, 139-149. 
Evdokimova, V., Ruzanov, P., Anglesio, M.S., Sorokin, A.V., Ovchinnikov, L.P., Buckley, J., Triche, T.J., Sonenberg, N., and Sorensen, P.H. (2006) Akt-mediated YB-1 phosphorylation activates translation of silent mRNA species. Mol Cell Biol. 26, 277-292.

Evdokimova, V., Ruzanov, P., Imataka, H., Raught, B., Svitkin, Y., Ovchinnikov, L.P., and Sonenberg N. (2001) The major mRNA-associated protein YB-1 is a potent 5' capdependent mRNA stabilizer. EMBO J. 20, 5491-5502.

Ewen, M.E., Faha, B., Harlow, E., Livingston, D.M. (1992) Interaction of p107 with cyclin A independent of complex formation with viral oncoproteins. Science. 255, 85-87.

Ewen, M.E., Lamb, J. (2004) The activities of cyclin D1 that drive tumorigenesis. Trends Mol Med. 10, 158-162.

Faha, B., Ewen, M.E., Tsai, L.H., Livingston, D.M., Harlow, E. (1992) Interaction between human cyclin A and adenovirus E1A-associated p107 protein. Science. 255, 8790 .

Fantl, V., Stamp, G., Andrews, A., Rosewell, I., Dickson, C. (1995) Mice lacking cyclin D1 are small and show defects in eye and mammary gland development. Genes Dev. 9, 2364-2372.

Frankel, P., Aronheim, A., Kavanagh, E., Balda, M.S., Matter, K., Bunney, T.D, Marshall, C.J. (2005) RalA interacts with ZONAB in a cell density-dependent manner and regulates its transcriptional activity. EMBO J. 24, 54-62.

Fraser, D.J., Phillips, A.O., Zhang, X, van Roeyen, C.R., Muehlenberg, P., En-Nia, A., Mertens, P.R. (2007) Y-box protein-1 controls transforming growth factor-beta1 translation in proximal tubular cells. Kidney Int. Dec 12; [Epub ahead of print]

Fu, M., Wang, C., Li, Z., Sakamaki, T., and Pestell, R.G. (2004) Cyclin D1: normal and abnormal functions. Endocrinology. 145, 5439-5447.

Fujii, T., Yokoyama, G., Takahashi, H., Namoto, R., Nakagawa, S., Toh, U., Kage, M., Shirouzu, K., Kuwano, M. (2008) Preclinical studies of molecular-targeting diagnostic and therapeutic strategies against breast cancer. Breast Cancer. 15, 73-78.

Fujita, T., Ito, K., Izumi, H., Kimura, M., Sano, M., Nakagomi, H., Maeno, K., Hama, Y., Shingu, K., Tsuchiya, S., Kohno, K., Fujimori, M. (2005) Increased nuclear localization of transcription factor Y-box binding protein 1 accompanied by up-regulation of Pglycoprotein in breast cancer pretreated with paclitaxel. Clin Cancer Res. 11, 8837-8844. 
Funke, B., Zuleger,B., Benavente, R., Schuster, T., Goller, M., Stévenin, J., Horak, I.(1996) The mouse poly(C)-binding protein exists in multiple isoforms and interacts with several RNA-binding proteins. Nucleic Acids Res. 24, 3821-3828.

Gaudreault, I., Guay, D., Lebel, M. (2004)YB-1 promotes strand separation in vitro of duplex DNA containing either mispaired bases or cisplatin modifications, exhibits endonucleolytic activities and binds several DNA repair proteins. Nucleic Acids Res. 32, 316-327.

Gessner, C., Woischwill, C., Schumacher, A., Liebers, U., Kuhn, H., Stiehl, P., Jürchott, K., Royer, H.D., Witt, C., Wolff, G. (2004) Nuclear YB-1 expression as a negative prognostic marker in nonsmall cell lung cancer. Eur Respir J. 23, 14-19.

Germain, D., Russell, A., Thompson, A., Hendley, J. (2000) Ubiquitination of free cyclin D1 is independent of phosphorylation on threonine 286. J Biol Chem. 275, 12074-12079.

Gillett, C., Fantl, V., Smith, R., Fisher, C., Bartek, J., Dickson, C., Barnes, D., Peters, G. (1994) Amplification and overexpression of cyclin D1 in breast cancer detected by immunohistochemical staining. Cancer Res. 54, 1812-1817.

Gladden, A.B., Diehl, J.A. (2005) Location, location, location: the role of cyclin D1 nuclear localization in cancer. J Cell Biochem. 96, 906-913.

Goujon, L., Allevato, G., Simonin, G., Paquereau, L., Le Cam, A., Clark, J., Nielsen, J.H., Djiane, J., Postel-Vinay, M.C., and Edery, M, et al. (1994) Cytoplasmic sequences of the growth hormone receptor necessary for signal transduction. Proc Natl Acad Sci U S A. 91, 957-961.

Grant, C.E., and Deeley, R.G. (1993) Cloning and characterization of chicken YB-1: regulation of expression in the liver. Mol Cell Biol. 13, 4186-4196.

Gu, C., Oyama, T., Osaki, T., Kohno, K., and Yasumoto, K. (2001) Expression of Y boxbinding protein-1 correlates with DNA topoisomerase Ilalpha and proliferating cell nuclear antigen expression in lung cancer. Anticancer Res. 21, 2357-2362.

Guay, D., Gaudreault, I., Massip, L., Lebel, M. (2006) Formation of a nuclear complex containing the 53 tumor suppressor, YB-1, and the Werner syndrome gene product in cells treated with UV light. Int J Biochem Cell Biol. 38, 1300-1313.

Guo, Y., Harwalkar, J., Stacey, D.W., Hitomi, M. (2005) Destabilization of cyclin D1 message plays a critical role in cell cycle exit upon mitogen withdrawal. Oncogene. 24, 1032-1042.

Guo, D., Hazbun, T.R., Xu, X.J., Ng, S.L., Fields, S., Kuo, M.H. (2004) A tethered catalysis, two-hybrid system to identify protein-protein interactions requiring posttranslational modifications. Nat Biotechnol. 22, 888-892. 
Guo, Y., Stacey, D.W., Hitomi, M.. (2002) Post-transcriptional regulation of cyclin D1 expression during G2 phase. Oncogene. 21, 7545-7556.

Guttridge, D.C., Albanese, C., Reuther, J.Y., Pestell, R.G., Baldwin, A.S. Jr. (1999) NFkappaB controls cell growth and differentiation through transcriptional regulation of cyclin D1. Mol Cell Biol. 19, 5785-5799.

Hall, M., and Peters, G.. (1996) Genetic alterations of cyclins, cyclin-dependent kinases, and Cdk inhibitors in human cancer. Adv Cancer Res. 68, 67-108.

Harlow, E., Whyte, P., Franza, B.R. Jr., Schley, C. (1986) Association of adenovirus early-region 1A proteins with cellular polypeptides. Mol Cell Biol.6, 1579-1589.

Hasegawa, S.L., Doetsch, P.W., Hamilton, K.K., Martin, A.M., Okenquist, S.A., Lenz, J., and Boss, J.M. (1991) DNA binding properties of YB-1 and dbpA: binding to doublestranded, single-stranded, and abasic site containing DNAs. Nucleic Acids Res. 19, 49154920.

Hashemolhosseini, S., Nagamine, Y., Morley, S.J., Desrivières, S., Mercep, L., Ferrari, S. (1998) Rapamycin inhibition of the G1 to $\mathrm{S}$ transition is mediated by effects on cyclin D1 mRNA and protein stability. J Biol Chem. 273, 14424-14429.

Higashi, K., Inagaki, Y., Suzuki, N., Mitsui, S., Mauviel, A., Kaneko, H., and Nakatsuka I. (2003) Y-box-binding protein YB-1 mediates transcriptional repression of human alpha 2(I) collagen gene expression by interferon-gamma. J Biol Chem. 278, 5156-5162.

Hipfel, R., Schittek, B., Bodingbauer, Y., and Garbe, C.. (2000) Specifically regulated genes in malignant melanoma tissues identified by subtractive hybridization. Br J Cancer. $82,1149-1157$.

Hitomi, M., Stacey, D.W. (1999) Cellular ras and cyclin D1 are required during different cell cycle periods in cycling NIH 3 T3 cells. Mol Cell Biol. 19, 4623-4632.

Hitomi, M., Stacey, D.W. (1999) Cyclin D1 production in cycling cells depends on ras in a cell-cycle-specific manner. Curr Biol. 9, 1075-1084.

Hitomi, M., Stacey, D.W. (2001) Ras-dependent cell cycle commitment during G2 phase. FEBS Lett. 490, 123-131.

Hiyama, H., Reeves, S.A. (1999) Role for cyclin D1 in UVC-induced and p53-mediated apoptosis. Cell Death Differ. 6, 565-569.

Holm, P.S., Bergmann, S., Jurchott, K., Lage, H., Brand, K., Ladhoff, A., Mantwill, K., Curiel, D.T., Dobbelstein, M., Dietel, M., Gansbacher, B., and Royer, H.D. (2002) YB-1 relocates to the nucleus in adenovirus-infected cells and facilitates viral replication by 
inducing E2 gene expression through the E2 late promoter. J Biol Chem. 277, 1042710434.

Holm, P.S., Lage, H., Bergmann, S., Jürchott, K., Glockzin, G., Bernshausen, A., Mantwill, K., Ladhoff, A., Wichert, A., Mymryk, J.S., Ritter, T., Dietel, M., Gänsbacher, B., Royer, H.D. (2004) Multidrug-resistant cancer cells facilitate E1-independent adenoviral replication: impact for cancer gene therapy. Cancer Res. 64, 322-328.

Horwitz, E.M., Maloney, K.A., Ley, T.J. (1994) A human protein containing a "cold shock" domain binds specifically to H-DNA upstream from the human gamma-globin genes. J Biol Chem. 269, 14130-14139.

Huang, J,, Tan, P.H., Li, K.B., Matsumoto, K., Tsujimoto, M., Bay, B.H. (2005) Y-box binding protein, YB-1, as a marker of tumor aggressiveness and response to adjuvant chemotherapy in breast cancer. Int J Oncol. 26, 607-613.

Ise, T., Nagatani, G., Imamura, T., Kato, K., Takano, H., Nomoto, M., Izumi, H., Ohmori, H., Okamoto, T., Ohga, T., Uchiumi, T., Kuwano, M., and Kohno, K. (1999) Transcription factor Y-box binding protein 1 binds preferentially to cisplatin-modified DNA and interacts with proliferating cell nuclear antigen. Cancer Res. 59, 342-346.

Janz, M., Harbeck, N., Dettmar, P., Berger, U., Schmidt, A., Jürchott, K., Schmitt, M., Royer, H.D. (2002) Y-box factor YB-1 predicts drug resistance and patient outcome in breast cancer independent of clinically relevant tumor biologic factors HER2, uPA and PAI-1. Int J Cancer. 97, 278-282.

Johnson, D.G., Walker, C.L. (1999) Cyclins and cell cycle checkpoints. Annu Rev Pharmacol Toxicol. 39, 295-312.

Jones, S.W., Christison, R., Bundell, K., Voyce, C.J., Brockbank, S.M., Newham, P., and Lindsay, M.A. (2005) Characterisation of cell-penetrating peptide-mediated peptide delivery. Br J Pharmacol. 145, 1093-1102.

Joyce, D., Bouzahzah, B., Fu, M., Albanese, C., D'Amico, M., Steer, J., Klein, J.U., Lee, R.J., Segall, J.E., Westwick, J.K., Der, C.J., Pestell, R.G. (1999) Integration of Racdependent regulation of cyclin D1 transcription through a nuclear factor-kappaBdependent pathway. J Biol Chem. 274, 25245-25249.

Jurchott, K., Bergmann, S., Stein, U., Walther, W., Janz, M., Manni, I., Piaggio, G., Fietze, E., Dietel, M., and Royer, H.D. (2003) YB-1 as a cell cycle-regulated transcription factor facilitating cyclin A and cyclin B1 gene expression. J Biol Chem. 278, 27988-27996.

Kamalov, G., Varma, B.R., Lu, L, Sun, Y., Weber, K.T., and Guntaka, R.V. (2005) Expression of the multifunctional Y-box protein, YB-1, in myofibroblasts of the infarcted rat heart.Biochem Biophys Res Commun. 334, 239-244. 
Kamura, T., Yahata, H., Amada, S., Ogawa, S., Sonoda, T., Kobayashi, H., Mitsumoto, M., Kohno, K., Kuwano, M., Nakano, H. (1999) Is nuclear expression of Y box-binding protein-1 a new prognostic factor in ovarian serous adenocarcinoma? Cancer 85, 24502454.

Kandala, J.C., and Guntaka, R.V. (1994) Cloning of Rous sarcoma virus enhancer factor genes. I. Evidence that RSV-EF-I is related to Y-box (inverted CCAAT) binding proteins and binds to multiple motifs in the RSV enhancer. Virology. 198, 514-523.

Kastan, M.B., Bartek, J. (2004) Cell-cycle checkpoints and cancer. Nature. 432, 316-323.

Klein, E.A., Yang, C., Kazanietz, M.G., Assoian, R.K. (2007) NFkappaB-independent signaling to the cyclin D1 gene by Rac. Cell Cycle. 6, 1115-1121.

Kohno, K., Izumi, H., Uchiumi, T., Ashizuka, M., and Kuwano, M. (2003) The pleiotropic functions of the Y-box-binding protein, YB-1. Bioessays. 25, 691-698.

Koike, K., Uchiumi, T., Ohga, T., Toh, S., Wada, M., Kohno, K., and Kuwano, M. (1997) Nuclear translocation of the Y-box binding protein by ultraviolet irradiation. FEBS Lett. 417, 390-394.

Kuriyan, J., Eisenberg, D. (2007) The origin of protein interactions and allostery in colocalization. Nature. 450, 983-990.

Kurosaki, T. Genetic analysis of B cell antigen receptor signaling. (1999) Annu Rev Immunol. 17, 555-592.

Kusano, K., Kagawa, N., Sakaguchi, M., Omura, T., and Waterman, M.R. (2001) Importance of a proline-rich sequence in the amino-terminal region for correct folding of mitochondrial and soluble microbial p450s. J Biochem (Tokyo). 129, 271-277.

Ladomery, M. (1997) Multifunctional proteins suggest connections between transcriptional and post-transcriptional processes. Bioessays. 19, 903-909.

Ladomery, M., and Sommerville, J. (1995) A role for Y-box proteins in cell proliferation. Bioessays. 17, 9-11.

Landsman, D. (1992) RNP-1, an RNA-binding motif is conserved in the DNA-binding cold shock domain. Nucleic Acids Res. 20, 2861-2864.

Lasham, A., Lindridge, E., Rudert, F., and Onrust, R., Watson, J. (2000) Regulation of the human fas promoter by YB-1, Puralpha and AP-1 transcription factors. Gene. 252, 113. 
Lee, R.J., Albanese, C., Fu, M., D'Amico, M., Lin, B., Watanabe, G., Haines, G.K. 3rd, Siegel, P.M., Hung, M.C., Yarden, Y., Horowitz, J.M., Muller, W.J., Pestell, R.G. (2000) Cyclin D1 is required for transformation by activated Neu and is induced through an E2F-dependent signaling pathway. Mol Cell Biol. 20, 672-683.

Lee, R.J., Albanese, C., Stenger, R.J., Watanabe, G., Inghirami, G., Haines, G.K. 3rd, Webster, M., Muller, W.J., Brugge, J.S., Davis, R.J., Pestell, R.G. (1999) pp60(v-src) induction of cyclin D1 requires collaborative interactions between the extracellular signal-regulated kinase, p38, and Jun kinase pathways. A role for cAMP response element-binding protein and activating transcription factor-2 in pp60(v-src) signaling in breast cancer cells. J Biol Chem. 274, 7341-7350.

Levitt, M. (1981) Effect of proline residues on protein folding. J Mol Biol. 145, 251-263.

Lewitt, M.S., Brismar, K., Ohlson, J., Hartman, J. (2001) Lithium chloride inhibits the expression and secretion of insulin-like growth factor-binding protein-1. J Endocrinol. 171, 11-15.

Li, W.W., Hsiung, Y., Wong, V., Galvin, K., Zhou, Y., Shi, Y., and Lee, A.S. (1997) Suppression of grp78 core promoter element-mediated stress induction by the dbpA and dbpB (YB-1) cold shock domain proteins. Mol Cell Biol. 17, 61-68.

Lin, S., Wang, W., Wilson, G.M., Yang, X., Brewer, G., Holbrook, N.J., Gorospe, M. (2000) Down-regulation of cyclin D1 expression by prostaglandin A(2) is mediated by enhanced cyclin D1 mRNA turnover. Mol Cell Biol. 20 7903-7913.

Lindquist, S. (1986) The heat-shock response. Annu Rev Biochem. 55, 1151-1191.

Lingle, W.L., Barrett, S.L., Negron, V.C., D'Assoro, A.B., Boeneman, K., Liu, W., Whitehead, C.M., Reynolds, C., Salisbury, J.L. (2002) Centrosome amplification drives chromosomal instability in breast tumor development. Proc Natl Acad Sci U S A. 99, 1978-1983.

Lu, Z.H., Books, J.T., Ley, T.J. (2005) YB-1 is important for late-stage embryonic development, optimal cellular stress responses, and the prevention of premature senescence. Mol Cell Biol. 25, 4625-4637.

Lukas, J., Bartkova, J., Bartek, J. (1996) Convergence of mitogenic signalling cascades from diverse classes of receptors at the cyclin D-cyclin-dependent kinase-pRb-controlled G1 checkpoint. Mol Cell Biol. 16, 6917-6925.

Lukas, J., Bartkova, J., Rohde, M., Strauss, M., Bartek, J. (1995) Cyclin D1 is dispensable for G1 control in retinoblastoma gene-deficient cells independently of cdk4 activity. Mol Cell Biol. 15, 2600-2611. 
Lukas, J., Jadayel, D., Bartkova, J., Nacheva, E., Dyer, M.J., Strauss, M., Bartek, J. (1994) BCL-1/cyclin D1 oncoprotein oscillates and subverts the G1 phase control in Bcell neoplasms carrying the $\mathrm{t}(11 ; 14)$ translocation. Oncogene. 9, 2159-2167.

MacDonald, G.H., Itoh-Lindstrom, Y., and Ting, J.P. (1995) The transcriptional regulatory protein, YB-1, promotes single-stranded regions in the DRA promoter. J Biol Chem. 270, 3527-3533.

Mantwill, K., Köhler-Vargas, N., Bernshausen, A., Bieler, A., Lage, H., Kaszubiak, A., Surowiak, P., Dravits, T., Treiber, U., Hartung, R., Gansbacher, B., Holm, P.S. (2006) Inhibition of the multidrug-resistant phenotype by targeting YB-1 with a conditionally oncolytic adenovirus: implications for combinatorial treatment regimen with chemotherapeutic agents. Cancer Res. 66, 7195-7202.

Marenstein, D.R., Ocampo, M.T., Chan, M.K., Altamirano A., Basu A.K., Boorstein, R.J., Cunningham, R.P., and Teebor, G.W. (2001) Stimulation of human endonuclease III by Y box-binding protein 1 (DNA-binding protein B). Interaction between a base excision repair enzyme and a transcription factor. J Biol Chem. 276, 21242-21249.

Marshall, C.J. (1999) Small GTPases and cell cycle regulation. Biochem Soc Trans. 27 363-370.

Marston, F.A.O. (1987) The purification of eukaryotic polypeptides expressed in Escherichia coli. In DNA cloning: A practical approach. 3, 59.

Marston, F.A.O., Lowe P.A.,, Doel, M.T., Schoemaker, J.M., White, S., Angal, S. (1984) Purification of calf prochymosin (prorennin)synthesized in Escherichia coli. Bio/Technology 2, 800 .

Massagué, J. (2004) G1 cell-cycle control and cancer. Nature. 432, 298-306.

Matsumoto, K., Meric, F., Wolffe, A.P. (1996) Translational repression dependent on the interaction of the Xenopus Y-box protein FRGY2 with mRNA. Role of the cold shock domain, tail domain, and selective RNA sequence recognition. J Biol Chem. 271, 2270622712 .

Matsumoto, K., Tanaka, K.J., and Tsujimoto, M. (2005) An acidic protein, YBAP1, mediates the release of YB-1 from mRNA and relieves the translational repression activity of YB-1. Mol Cell Biol. 25, 1779-1792.

Matsumoto, K., Wolffe, A.P. (1998) Gene regulation by Y-box proteins: coupling control of transcription and translation. Trends Cell Biol. 8, 318-323.

Matsumura, I., Kitamura, T., Wakao, H., Tanaka, H., Hashimoto, K., Albanese, C., Downward, J., Pestell, R.G., Kanakura, Y. (1999) Transcriptional regulation of the cyclin 
D1 promoter by STAT5: its involvement in cytokine-dependent growth of hematopoietic cells. EMBO J. 18, 1367-1377.

Matsushime, H., Ewen, M.E., Strom, D.K., Kato, J.Y., Hanks, S.K., Roussel, M.F., Sherr, C.J. (1992) Identification and properties of an atypical catalytic subunit (p34PSKJ3/cdk4) for mammalian D type G1 cyclins. Cell. 71, 323-334.

Matsushime, H., Roussel, M.F., Ashmun, R.A., Sherr, C.J. (1991) Colony-stimulating factor 1 regulates novel cyclins during the G1 phase of the cell cycle. Cell. 65, 701-713.

Mertens, P.R., Alfonso-Jaume, M.A., Steinmann, K., and Lovett, D.H. (1999) YB-1 regulation of the human and rat gelatinase A genes via similar enhancer elements. J Am Soc Nephrol. 10, 2480-2487.

Mertens, P.R., Harendza S., Pollock A.S., and Lovett D.H. (1997) Glomerular mesangial cell-specific transactivation of matrix metalloproteinase 2 transcription is mediated by YB-1. J Biol Chem. 272, 22905-22912.

Mertens, P.R., Steinmann, K., Alfonso-Jaume, M.A., En-Nia, A., Sun, Y., and Lovett, D.H. (2002) Combinatorial interactions of p53, activating protein-2, and YB-1 with a single enhancer element regulate gelatinase A expression in neoplastic cells. J Biol Chem. 277, 24875-24882.

Meyerson, M., Harlow, E. (1994) Identification of G1 kinase activity for cdk6, a novel cyclin D partner. Mol Cell Biol. 14, 2077-2086.

Michalides, R., Hageman, P., van Tinteren, H., Houben, L., Wientjens, E., Klompmaker, R., Peterse, J. (1996) A clinicopathological study on overexpression of cyclin D1 and of p53 in a series of 248 patients with operable breast cancer. Br J Cancer. 73, 728-734.

Minich, W.B., and Ovchinnikov, L.P. (1992) Role of cytoplasmic mRNP proteins in translation. Biochimie. 74, 477-483.

Miyakawa, Y., Matsushime, H. (2001) Rapid downregulation of cyclin D1 mRNA and protein levels by ultraviolet irradiation in murine macrophage cells. Biochem Biophys Res Commun. 284 71-76.

Montani, V., Taniguchi, S.I., Shong, M., Suzuki, K., Ohmori, M., Giuliani, C., Napolitano, G., Saji, M., Fiorentino, B., Reimold, A.M., Ting, J.P., Kohn, L.D., and Singer, D.S. (1998) Major histocompatibility class II HLA-DR alpha gene expression in thyrocytes: counter regulation by the class II transactivator and the thyroid Y box protein. Endocrinology. 139, 280-289.

Moorthamer, M., Zumstein-Mecker, S., Chaudhuri, B. (1999) DNA binding protein dbpA binds Cdk5 and inhibits its activity. FEBS Lett. 446, 343-350. 
Moraes, K.C., Quaresma, A.J., Maehnss, K., Kobarg, J. (2003) Identification and characterization of proteins that selectively interact with isoforms of the mRNA binding protein AUF1 (hnRNP D). Biol Chem. 384, 25-37.

Motokura, T., Bloom, T., Kim, H.G., Jüppner, H., Ruderman, J.V., Kronenberg, H.M., Arnold, A. (1991) A novel cyclin encoded by a bcl1-linked candidate oncogene. Nature. $350,512-515$.

Murray, M.T, Schiller, D.L., Franke, W.W. (1992) Sequence analysis of cytoplasmic mRNA-binding proteins of Xenopus oocytes identifies a family of RNA-binding proteins. Proc Natl Acad Sci U S A. 89, 11-15.

Nagata,D. Suzuki,E. Nishimatsu,H. Satonaka,H. Goto,A. Omata,M. Hirata,Y. (2001) Transcriptional activation of the cyclin D1 gene is mediated by multiple cis-elements, including SP1 sites and a cAMP-responsive element in vascular endothelial cells. J Biol Chem. 276, 662-669.

Nakayama, T., and Takami, Y. (2001) Participation of histones and histone-modifying enzymes in cell functions through alterations in chromatin structure. J Biochem. 129, 491-499.

Nekrasov, M.P., Ivshina, M.P., Chernov, K.G., Kovrigina, E.A., Evdokimova, V.M., Thomas, A.A., Hershey, J.W., and Ovchinnikov, L.P. (2003) The mRNA-binding protein YB-1 (p50) prevents association of the eukaryotic initiation factor eIF4G with mRNA and inhibits protein synthesis at the initiation stage. J Biol Chem. 278, 13936-13943.

Nichols, G.E., Williams, M.E., Gaffey, M.J., Stoler, M.H. (1996) Cyclin D1 gene expression in human cervical neoplasia. Mod Pathol. 9, 418-425.

Nigg, E.A. (2002) Centrosome aberrations: cause or consequence of cancer progression? Nat Rev Cancer. 2, 815-825.

Norman, J.T., Lindahl ,G.E., Shakib, K., En-Nia, A., Yilmaz, E., and Mertens, P.R. (2001) The Y-box binding protein YB-1 suppresses collagen alpha 1(I) gene transcription via an evolutionarily conserved regulatory element in the proximal promoter. J Biol Chem. 276, 29880-29890.

Oda, Y., Sakamoto, A., Shinohara, N., Ohga, T., Uchiumi, T., and Kohno, K., Tsuneyoshi, M., Kuwano, M., Iwamoto, Y. (1998) Nuclear expression of YB-1 protein correlates with P-glycoprotein expression in human osteosarcoma .Clin Cancer Res. 4, 2273-2277.

Oda, Y., Ohishi, Y., Saito, T., Hinoshita, E., Uchiumi, T., Kinukawa, N., Iwamoto, Y., Kohno, K., Kuwano, M., Tsuneyoshi, M. (2003) Nuclear expression of Y-box-binding protein-1 correlates with P-glycoprotein and topoisomerase II alpha expression, and with poor prognosis in synovial sarcoma. J Pathol. 199, 251-258. 
Ohga, T., Koike, K., Ono, M., Makino, Y., Itagaki, Y., Tanimoto, M., Kuwano, M., and Kohno, K. (1996) Role of the human Y box-binding protein YB-1 in cellular sensitivity to the DNA-damaging agents cisplatin, mitomycin C, and ultraviolet light. Cancer Res. $56,4224-4228$.

Ohga, T., Uchiumi, T., Makino, Y., Koike, K., Wada, M., Kuwano, M., and Kohno, K. (1998) Direct involvement of the Y-box binding protein YB-1 in genotoxic stressinduced activation of the human multidrug resistance 1 gene. J Biol Chem. 273, 59976000 .

Ohmori, M., Shimura, H., Shimura, Y., Kohn, L.D. (1996) A Y-box protein is a suppressor factor that decreases thyrotropin receptor gene expression. Mol Endocrinol. $10,76-89$.

Ohtsubo, M., Theodoras, A.M., Schumacher, J., Roberts, J.M., Pagano, M. (1995) Human cyclin E, a nuclear protein essential for the G1-to-S phase transition. Mol Cell Biol. 15 2612-2624.

Okamoto, T., Izumi, H., Imamura, T., Takano, H., Ise, T., Uchiumi, T., Kuwano, M., and Kohno, K. (2000) Direct interaction of p53 with the Y-box binding protein, YB-1: a mechanism for regulation of human gene expression. Oncogene. 19, 6194-6202.

Osváth, S., Gruebele, M. (2003) Proline can have opposite effects on fast and slow protein folding phases. Biophys J. 85, 1215-1222.

Palmero, I., Peters, G. P(1996) erturbation of cell cycle regulators in human cancer. Cancer Surv. 27, 351-367.

Paranjape, S.M., Harris, E. (2007) Y box-binding protein-1 binds to the dengue virus 3'untranslated region and mediates antiviral effects. J Biol Chem. 282, 30497-30508. Patterson, S.D., Aebersold, R.H. (2003) Proteomics: the first decade and beyond. Nat Genet 33 311-323.

Pawson, T. (2004) Specificity in signal transduction: from phosphotyrosine-SH2 domain interactions to complex cellular systems. Cell. 116 191-203.

Peters, G. (1994) The D-type cyclins and their role in tumorigenesis. J Cell Sci Suppl. $18,89-96$.

Piel, M., Nordberg, J., Euteneuer, U., Bornens, M. (2001) Centrosome-dependent exit of cytokinesis in animal cells. Science. 291, 1550-1553.

Phizicky, E.M., Fields, S. (1995) Protein-protein interactions: methods for detection and analysis. Microbiol Rev. 59, 94-123. 
Prochiantz, A. (1996) Getting hydrophilic compounds into cells: lessons from homeopeptides. Curr Opin Neurobiol. 6, 629-634.

Proctor, A.J., Coombs, L.M., Cairns, J.P., Knowles, M.A. (1991) Amplification at chromosome 11q13 in transitional cell tumours of the bladder. Oncogene. 6, 789-795.

Raffetseder, U., Frye, B., Rauen, T., Jürchott, K., Royer, H.D., Jansen, P.L., and Mertens, P.R. (2003) Splicing factor SRp30c interaction with Y-box protein-1 confers nuclear YB1 shuttling and alternative splice site selection. J Biol Chem. 278, 18241-18248.

Richard, J.P., Melikov, K., Vives, E., Ramos, C., Verbeure, B., Gait, M.J., Chernomordik, L.V., and Lebleu, B. (2003) Cell-penetrating peptides. A reevaluation of the mechanism of cellular uptake. J Biol Chem. 278, 585-590.

Robinson, D.N., Spudich, J.A. (2004) Mechanics and regulation of cytokinesis. Curr Opin Cell Biol. 16, 182-188.

Rosenthal, E.T., Hunt, T., Ruderman, J.V. (1980) Selective translation of mRNA controls the pattern of protein synthesis during early development of the surf clam, Spisula solidissima. Cell. 20, 487-494.

Rosenwald, I.B., Kaspar, R., Rousseau, D., Gehrke, L., Leboulch, P., Chen, J.J., Schmidt, E.V., Sonenberg, N., London, I.M. (1995) Eukaryotic translation initiation factor 4E regulates expression of cyclin D1 at transcriptional and post-transcriptional levels. J Biol Chem. $27021176-21180$.

Roussel, M.F., Theodoras, A.M., Pagano, M., Sherr, C.J. (1995 )Rescue of defective mitogenic signaling by D-type cyclins. Proc Natl Acad Sci U S A. 92, 6837-6841.

Ruzanov, P.V., Evdokimova, V.M., Korneeva, N.L., Hershey, J.W., Ovchinnikov, L.P. (1999) Interaction of the universal mRNA-binding protein, p50, with actin: a possible link between mRNA and microfilaments. J Cell Sci. 112, 3487-3496.

Sabath, D.E., Podolin, P.L., Comber, P.G., and Prystowsky, M.B. (1990) cDNA cloning and characterization of interleukin 2-induced genes in a cloned T helper lymphocyte. $\mathrm{J}$ Biol Chem. 265, 12671-12678.

Safak, M., Gallia, G.L., Ansari, S.A., and Khalili, K. (1999) Physical and functional interaction between the Y-box binding protein YB-1 and human polyomavirus JC virus large T antigen. J Virol. 73, 10146-10157.

Saji, H., Toi, M., Saji, S., Koike, M., Kohno, K., Kuwano, M. (2003) Nuclear expression of YB-1 protein correlates with P-glycoprotein expression in human breast carcinoma. Cancer Lett. 190, 191-197. 
Samuel, S., Beifuss, K.K., Bernstein, L.R. (2007) YB-1 binds to the MMP-13 promoter sequence and represses MMP-13 transactivation via the AP-1 site. Biochim Biophys Acta. 69, 525-531.

Sawaya, B.E., Khalili, K., and Amini, S. (1998) Transcription of the human immunodeficiency virus type 1 (HIV-1) promoter in central nervous system cells: effect of YB-1 on expression of the HIV-1 long terminal repeat. J Gen Virol. 79, 239-246.

Schindelin, H., Marahiel, M.A., Heinemann, U. (1993) Universal nucleic acid-binding domain revealed by crystal structure of the B. subtilis major cold-shock protein. Nature. 364, 164-168.

Schittek, B., Psenner, K., Sauer, B., Meier, F., Iftner, T., and Garbe, C. (2007) The increased expression of $\mathrm{Y}$ box-binding protein 1 in melanoma stimulates proliferation and tumor invasion, antagonizes apoptosis and enhances chemoresistance. Int J Cancer $120,2110-2118$.

Semczuk, A., Jakowicki, J.A. (2004) Alterations of pRb1-cyclin D1-cdk4/6-p16(INK4A) pathway in endometrial carcinogenesis. Cancer Lett. 203, 1-12.

Shaywitz, A.J., Dove, S.L., Greenberg, M.E., Hochschild, A. (2002) Analysis of phosphorylation-dependent protein-protein interactions using a bacterial two-hybrid system. Sci STKE. 20022311.

Sherr, C.J. (1996) Cancer cell cycles. Science. 274, 1672-1677.

Sherr, C.J. (2000) Cell cycle control and cancer. Harvey Lect. 96, 73-92.

Sherr, C.J., Matsushime, H., Roussel, M.F. (1992) Regulation of CYL/cyclin D genes by colony-stimulating factor 1. Ciba Found Symp. 170, 209-219.

Sherr, C.J., McCormick, F. (2002) The RB and p53 pathways in cancer. Cancer Cell. 2, 103-112.

Sherr, C.J., Roberts, J.M. (1999) CDK inhibitors: positive and negative regulators of G1phase progression. Genes Dev. 13, 1501-1512.

Shibahara, K., Sugio, K., Osaki, T., Uchiumi, T., Maehara, Y., Kohno, K., Yasumoto, K., Sugimachi, K., and Kuwano, M. (2001) Nuclear expression of the Y-box binding protein, YB-1, as a novel marker of disease progression in non-small cell lung cancer. Clin Cancer Res. 7, 3151-3155.

Shibahara, K., Uchiumi, T., Fukuda, T., Kura, S., Tominaga, Y., Maehara, Y., Kohno, K., and Nakabeppu Y., Tsuzuki T., and Kuwano M. (2004) Targeted disruption of one allele of the Y-box binding protein-1 (YB-1) gene in mouse embryonic stem cells and increased sensitivity to cisplatin and mitomycin C. Cancer Sci. 95, 348-353. 
Shibao, K., Takano, H., Nakayama, Y., Okazaki, K., Nagata, N., Izumi, H., Uchiumi, T., Kuwano, M., Kohno, K., and Itoh, H. (1999) Enhanced coexpression of YB-1 and DNA topoisomerase II alpha genes in human colorectal carcinomas. Int J Cancer. 83, 732-737.

Shnyreva, M., Schullery, D.S., Suzuki, H., Higaki, Y., Bomsztyk, K. (2000) Interaction of two multifunctional proteins. Heterogeneous nuclear ribonucleoprotein $\mathrm{K}$ and $\mathrm{Y}$-boxbinding protein. J Biol Chem. 275, 15498-15503.

Sicinski, P., Donaher, J.L., Parker, S.B., Li, T., Fazeli, A., Gardner, H., Haslam, S.Z., Bronson, R.T, Elledge, S.J., Weinberg, R.A. (1995) Cyclin D1 provides a link between development and oncogenesis in the retina and breast. Cell. 82, 621-630.

Skabkin, M.A., Evdokimova, V., Thomas, A.A., Ovchinnikov, L.P. (2001) The major messenger ribonucleoprotein particle protein p50 (YB-1) promotes nucleic acid strand annealing. J Biol Chem. 276, 44841-44847.

Skabkin, M.A., Kiselyova, O.I., Chernov, K.G., Sorokin, A.V., Dubrovin, E.V., Yaminsky, I.V., Vasiliev, V.D., and Ovchinnikov, L.P. (2004) Structural organization of mRNA complexes with major core mRNP protein YB-1. Nucleic Acids Res. 32, 56215635.

Skabkina, O.V., Lyabin, D.N., Skabkin M.A., and Ovchinnikov, L.P. (2005) YB-1 autoregulates translation of its own mRNA at or prior to the step of $40 \mathrm{~S}$ ribosomal subunit joining. Mol Cell Biol. 25, 3317-3323.

Skabkina, O.V., Skabkin, M.A., Popova, N.V., Lyabin, D.N., Penalva, L.O., and Ovchinnikov, L.P. (2003) Poly(A)-binding protein positively affects YB-1 mRNA translation through specific interaction with YB-1 mRNA. J Biol Chem. 278, 1819118198.

Sommerville, J. (1999) Activities of cold-shock domain proteins in translation control. Bioessays. 21, 319-325.

Soop, T., Nashchekin, D., Zhao, J., Sun, X., Alzhanova-Ericsson, A.T., Björkroth, B., Ovchinnikov, L., Daneholt, B. (2003) A p50-like Y-box protein with a putative translational role becomes associated with pre-mRNA concomitant with transcription. $\mathrm{J}$ Cell Sci. 116, 1493-1503.

Stacey, D.W. (2003) Cyclin D1 serves as a cell cycle regulatory switch in actively proliferating cells. Curr Opin Cell Biol. 15, 158-163.

Stein, U., Bergmann, S., Scheffer, G.L., Scheper, R.J., Royer, H.D., Schlag, P.M., Walther, W. (2005) YB-1 facilitates basal and 5-fluorouracil-inducible expression of the human major vault protein (MVP) gene. Oncogene. 24, 3606-3618. 
Stein, U., Jurchott, K., Walther, W., Bergmann, S., Schlag, P.M., and Royer, H.D. (2001) Hyperthermia-induced nuclear translocation of transcription factor YB-1 leads to enhanced expression of multidrug resistance-related ABC transporters. J Biol Chem. 276, 28562-28569.

Stenina, O.I., Shaneyfelt, K.M., and DiCorleto, P.E. (2001) Thrombin induces the release of the Y-box protein dbpB from mRNA: a mechanism of transcriptional activation. Proc Natl Acad Sci U S A. 98, 7277-7282.

Stickeler, E., Fraser, S.D., Honig, A., Chen, A.L., Berget, S.M., and Cooper, T.A. (2001) The RNA binding protein YB-1 binds A/C-rich exon enhancers and stimulates splicing of the CD44 alternative exon v4. EMBO J. 20, 3821-3830.

Stratford, A.L., Habibi, G., Astanehe, A., Jiang, H., Hu, K., Park, E., Shadeo, A., Buys, T.P., Lam, W., Pugh, T., Marra, M., Nielsen, T.O., Klinge, U., Mertens, P.R., Aparicio, S., Dunn, S.E. (2007) Epidermal growth factor receptor (EGFR) is transcriptionally induced by the Y-box binding protein-1 (YB-1) and can be inhibited with Iressa in basallike breast cancer, providing a potential target for therapy. Breast Cancer Res. 9, R61.

Sumrejkanchanakij, P., Tamamori-Adachi, M., Matsunaga, Y., Eto, K., Ikeda, M.A. (2003) Role of cyclin D1 cytoplasmic sequestration in the survival of postmitotic neurons. Oncogene. 22, 23-87.

Sutherland, B.W., Kucab, J., Wu, J., Lee, C., Cheang M.C., Yorida E., Turbin, D., Dedhar, S., Nelson, C., Pollak, M., Leighton Grimes, H., Miller, K., Badve, S., Huntsman, D., Blake-Gilks, C., Chen, M., Pallen, C.J., and Dunn, S.E. (2005) Akt phosphorylates the Y-box binding protein 1 at Ser102 located in the cold shock domain and affects the anchorage-independent growth of breast cancer cells. Oncogene. 24, 4281-4292.

Swaminathan, S.K., Nambiar, A., and Guntaka, R.V. (1997) Chicken YB-2, a Y-box protein, is a potent activator of Rous sarcoma virus long terminal repeat-driven transcription in avian fibroblasts. J Virol. 71, 2873-2880.

Swamynathan, S.K., Nambiar, A., and Guntaka, R.V. (1998) Role of single-stranded DNA regions and $\mathrm{Y}$-box proteins in transcriptional regulation of viral and cellular genes. FASEB J. 12, 515-522.

Swamynathan, S.K, Varma, B.R., Weber, K.T., and Guntaka, R.V. (2002) Targeted disruption of one allele of the Y-box protein gene, Chk-YB-1b, in DT40 cells results in major defects in cell cycle. Biochem Biophys Res Commun. 296, 451-457.

Tafuri, S.R., Wolffe, A.P. (1992) DNA binding, multimerization, and transcription stimulation by the Xenopus Y box proteins in vitro. New Biol. 4, 349-359. 
Tafuri, S.R., Wolffe, A.P. (1993) Selective recruitment of masked maternal mRNA from messenger ribonucleoprotein particles containing FRGY2 (mRNP4). J Biol Chem. 268, 24255-24261.

Takami, Y., Takeda, S., and Nakayama, T. (1997) An approximately half set of histone genes is enough for cell proliferation and a lack of several histone variants causes protein pattern changes in the DT40 chicken B cell line. J Mol Biol. 265, 394-408.

Takeda, S., Masteller, E.L., Thompson, C.B., and Buerstedde, J.M. (1992) RAG-2 expression is not essential for chicken immunoglobulin gene conversion. Proc Natl Acad Sci U S A. 89, 4023-4027.

Tamamori-Adachi, M., Ito, H., Sumrejkanchanakij, P., Adachi, S., Hiroe, M., Shimizu, M., Kawauchi, J., Sunamori, M., Marumo, F., Kitajima, S., Ikeda, M.A. (2003) Critical role of cyclin D1 nuclear import in cardiomyocyte proliferation. Circ Res. 92, 12-19.

Thieringer, H.A., Jones, P.G., Inouye, M. (1998) Cold shock and adaptation. Bioessays. 20, 49-57.

Topisirovic, I., Culjkovic. B., Cohen, N., Perez, J.M., Skrabanek, L., Borden, K.L. (2003) The proline-rich homeodomain protein, $\mathrm{PRH}$, is a tissue-specific inhibitor of eIF4Edependent cyclin D1 mRNA transport and growth. EMBO J. 22, 689-703.

Topisirovic, I., Guzman, M.L., McConnell, M.J., Licht, J.D., Culjkovic, B., Neering, S.J., Jordan, C.T., Borden, K.L. (2003) Aberrant eukaryotic translation initiation factor 4Edependent mRNA transport impedes hematopoietic differentiation and contributes to leukemogenesis. Mol Cell Biol. 23, 8992-9002.

Uchiumi, T., Fotovati, A., Sasaguri, T., Shibahara, K., Shimada T., Fukuda, T., Nakamura, T., Izumi, H., and Tsuzuki T., Kuwano, M., Kohno, K. (2006) YB-1 is important for an early stage embryonic development: neural tube formation and cell proliferation. J Biol Chem. 281, 40440-40449.

Um, M., Yamauchi, J., Kato, S., and Manley, J.L. (2001) Heterozygous disruption of the TATA-binding protein gene in DT40 cells causes reduced cdc25B phosphatase expression and delayed mitosis. Mol Cell Biol. 21, 2435-2448.

Uramoto, H., Izumi, H., Ise, T., Tada, M., Uchiumi, T., Kuwano, M., Yasumoto, K., Funa, K., Kohno, K. (2002) p73 Interacts with c-Myc to regulate Y-box-binding protein1 expression. J Biol Chem. 277, 31694-31702.

Vaiman, A.V., Stromskaya, T.P., Rybalkina, E.Y., Sorokin, A.V., Ovchinnikov, L.P., Stavrovskaya, A.A. (2007) Development of drug resistance in the population of colon cancer cells under the effect of multifunctional protein YB-1. Bull Exp Biol Med. 143, 463-466. 
Vidal, M., Gigoux, V., and Garbay, C. (2001) SH2 and SH3 domains as targets for antiproliferative agents. Crit Rev Oncol Hematol. 40, 175-186.

Wang, J., Takagaki, Y., and Manley, J.L. (1996) Targeted disruption of an essential vertebrate gene: ASF/SF2 is required for cell viability. Genes Dev. 10, 2588-2599.

Watanabe, G., Albanese, C., Lee, R.J., Reutens, A., Vairo, G., Henglein, B., Pestell, R.G. (1998) Inhibition of cyclin D1 kinase activity is associated with E2F-mediated inhibition of cyclin D1 promoter activity through E2F and Sp1. Mol Cell Biol. 18, 3212-3222.

Watanabe, G., Howe, A., Lee, R.J., Albanese, C., Shu, I.W., Karnezis, A.N., Zon, L., Kyriakis, J., Rundell, K., Pestell, R.G. (1996) Induction of cyclin D1 by simian virus 40 small tumor antigen. Proc Natl Acad Sci U S A. 93, 12861-12866.

Wilusz, C.J., Wormington, M., Peltz, S.W. (2001) The cap-to-tail guide to mRNA turnover. Nat Rev Mol Cell Biol. 2, 237-246.

Winston, J., Dong, F., Pledger, W.J. (1996) Differential modulation of G1 cyclins and the Cdk inhibitor p27kip1 by platelet-derived growth factor and plasma factors in density-arrested fibroblasts. J Biol Chem. 271, 11253-11260.

Winston, J.T., Coats, S.R., Wang, Y.Z., Pledger, W.J. (1996) Regulation of the cell cycle machinery by oncogenic ras. Oncogene. 12, 127-134.

Wistow, G. (1990) Cold shock and DNA binding. Nature. 344, 823-824.

Wolffe, A.P. (1994) Nucleosome positioning and modification: chromatin structures that potentiate transcription. Trends Biochem Sci. 19, 240-244.

Wolffe, A.P. (1994) The transcription of chromatin templates. Curr Opin Genet Dev. 4, 245-254.

Wolffe, A.P., Tafuri, S., Ranjan, M., Familari, M. (1992) The Y-box factors: a family of nucleic acid binding proteins conserved from Escherichia coli to man. New Biol. 4, 290298.

Worsley, S.D., Ponder, B.A., Davies, B.R. (1997) Overexpression of cyclin D1 in epithelial ovarian cancers. Gynecol Oncol. 64, 189-195.

Xiong, Y., Connolly, T., Futcher, B., Beach, D. (1991) Human D-type cyclin. Cell. 65, 691-699.

Yahata, H., Kobayashi, H., Kamura, T., Amada, S., Hirakawa, T., Kohno, K., Kuwano, M., Nakano, H. (2002) Increased nuclear localization of transcription factor YB-1 in acquired cisplatin-resistant ovarian cancer. J Cancer Res Clin Oncol. 128, 621-626. 
Yasen, M., Kajino, K., Kano, S., Tobita, H., Yamamoto, J., Uchiumi, T., Kon, S., Maeda, M., Obulhasim, G., Arii, S., Hino, O. (2005) The up-regulation of Y-box binding proteins (DNA binding protein A and Y-box binding protein-1) as prognostic markers of hepatocellular carcinoma. Clin Cancer Res. 11, 7354-7361.

Zhang, W.W., Huang, H.F., Li, Q.W., Ma, F. (2006) Functions of Y-box binding protein and its role in tumorigenicity Yi Chuan. 28, 1153-1160.

Zhou, F., Galan, J., Geahlen, R.L., Tao, W.A. (2007) A novel quantitative proteomics strategy to study phosphorylation-dependent peptide-protein interactions. J Proteome Res. 6, 133-140.

Zou, Y., and Chien, K.R. (1995) EFIA/YB-1 is a component of cardiac HF-1A binding activity and positively regulates transcription of the myosin light-chain $2 \mathrm{v}$ gene. Mol Cell Biol. 15, 2972-2982.

Zou, Y., Ewton, D.Z., Deng, X., Mercer, S.E., Friedman, E. (2004) Mirk/dyrk1B kinase destabilizes cyclin D1 by phosphorylation at threonine 288. J Biol Chem. 279, 2779027798. 


\section{Vita}

Payal Khandelwal was born in Lonavala (Pune), India in 1979. She graduated from Hyderabad Central University with Masters in Biotechnology in June 2002. She enrolled in the Ph.D. program in Molecular Sciences at University of Tennessee Health Science Center in the fall of 2002 and worked on the role of YB-1 in Cell proliferation and Apoptosis in the laboratory of Dr. Ramareddy V. Guntaka. She graduated from UTHSC in May 2008. 